\title{
PROLEGOMENA CRITICA
}

Vix ulli alii operi antiquitatis id contigit, quod in Elementis Euclidis factum videmus, ut inde a primo tempore, quo editum sit, ad nostrum usque aevum idoneum haberetur, quod proposito suo satisfaceret. constat enim, Euclidem in Elementis hoc sibi proposuisse, ut artem, quam vocant, mathematicam scriberet, unde huius scientiae studiosi solida doctrinae initia et apta fundamenta ad difficiliores gradus scientiac aggrediendos caperent. et liber eins statim tanto favore exceptus est, ut ceteros libros eiusdem generis, inter quos Elementa Theudii non ita multo ante edita erant, prorsus obscuraret et ex usu manibusque hominum removeret; ad nos saltim nihil fere nisi nomina et brevissima notitia eorum pervenit (Proclus in Eucl. p. 66sq. ex Eudemo). quare videmus, reliquos mathematicos Graecos ad unum omnes ad Elementa Euclidis appellare, rebus in iis demonstratis tamquam certis omnibusque notis uti, hoc fundamento sua opera instruere, sicut iam Proclus p. $71,17 \mathrm{sq}$. recte observavit. etiam iis, qui mathematicam professi non essent, satis notum fuisse hoc opus apparet ex locis plurimis, quibus nominatur et citatur, ubi occasio rerum mathematicarum commemorandarum scriptoribus non mathematicis oblata est, quorum locorum potiores posui in libro, qui inscribitur Literargeschichtliche Studien über Euklid p. 30 et p. 193 sq. et hodie quoque pueri et in Britannia et in Suecia et aliis locis primam mathematices notitiam ex hoc libro venerabili hauriunt; Britannis quidem nomen Euclidis prope in appellativum cessit.

Per tam longum temporis spatium fieri non potuit, quin multa mutarentur et sensim a pristina operis forma declinarent, quamquam propter res vel mediocriter doctis notas et perspicuas cer. tisque quasi formulis expositas minus quam alibi in hoc opere describendo peccaverunt librarii. rursus autem, cum Elementa manibus magistrorum et discipulorum tererentur, multis locis interpolabantur, quae docentibus discentibusque ad verba Euclidis explicanda utilia videbantur, quod idem in omnibus eius modi operibus antiquitatis factum videmus velut in libris grammaticorum, in lexicis cet. itaque ut tempus et genera harum interpolationum distinguamus, quod vel praecipuum opus ei est, qui fata Elementorum persequi velit, ante omnia necesse est, ut certum aliquod fundamentum quaeramus, unde disquisitio nostra in utramque partem exire possit. quare ab editione Theonis incipiendum esse putavi. sed prius quam de singulis disputamus, ostendendum, qua auctoritate in ea restituenda nitamur. 


\section{PROLEGOMENA CRITICA}

\section{Cap. I}

\section{Quibus auctoribus de editione Theonis iadicari possit}

Cardo huius quaestionis in loco illo memorabili commentariorum Theonis in Ptolemaeum est, ubi legimus (I p. 201 ed. Halma

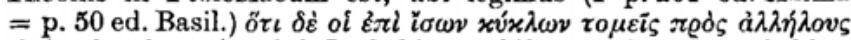

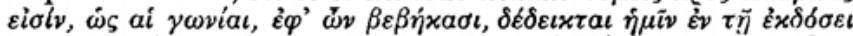

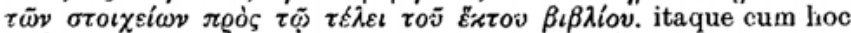
additamentum paene omnes codices nostri in VI 33 habeant, e recensione Theonis profecti sunt, id quod plerumque ipsi titulis suis

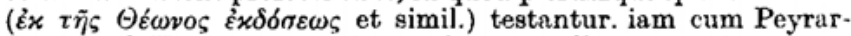
dus in cod. Vat. 190 neque interpolationem illam neque hunc titu. lum inveniret, suo iure hunc codicem recensionem Theone antiquiorem continere iudicavit. et librarium codicis $\mathbf{P}$ sive potius archetypi eius duas illas recensiones novisse et dedita opera antiquam praetulisse, apparet e scholio memorabili, quod IV p. 144

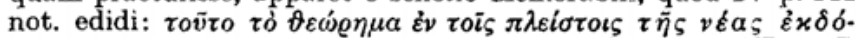

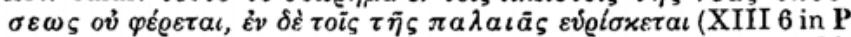
exstat, in nonnullis Theoninis deest). itaque comparatis codd. Theoninis et $\mathbf{P}$ de mutationibus a Theone factis in universum iudicare possumus.

In m primum ad brevem notitiam codicis Vat. 190, quam dedi I p. VIII, uberiorem descriptionem adiungnm.

Codex Vaticanus igitur graecus numero 190 signatus, membran. forma 4ta, nunc duobus constat voluminibus, quae sine dubio olim coniuncta erant. codex ipse, qui saeculo $\mathrm{X}$ tribuendus est, totus eadem manu nitida et accurata scriptus est litteris oblongis, atramento badio. in singulis paginis binae columnae. spiritus accentusque plerumque deerant, multis locis manibus recentioribus additi sunt, sed inconstanter (in libris XIV et XV et in scholiis prorsus omittuntur). correcturae aliae manu prima factae sunt, sed plerumque atramento pallidiore, aliae manu recentissima ( $\mathbf{P}$ m. rec.), aliae compluribus manibus satis antiquis vel eadem manu alibi alio atramento (P m. 2). continet fol. $1-2$ indicem totius co. dicis; deinde sequuntur duo folia chartacea sine numeris, de quibus Peyrardus adscripsit ,,ceci est un déchiffrement du commencement de ce qui suit sur parchemin"; continent, quae infra edidi p. 39, 2

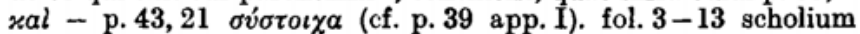
nostrum I I; haec 11 folia membran. numeros antiquos non habent, sed numerata sunt manu hodierna, sicut totus codex. fol. 14-174 Elem. I-X 86 in quaternionibus XIX a manu 1 numeris $\bar{a}-\overline{t \vartheta}$ in summo margine dextro primi folii signatis. initio saepe folia membran. foliorumve partes assuta sunt, quae in numero paginarum computantur, in quaternionibus non computantur; continent scholia m. 2.-vol. II fol. 175-247 Elem. X 87-XIII. fol. 248-249 Marini comm. in Data sine auctoris nomine, iisdem litteris deminutis scriptum, quibus manus prima in scholiis utitur. fol. 250-281 Data. fol. 282 scholia in Data litteris minoribus. 


\section{PROLEGOMENA CRITICA}

fol. 283-292 Elem. XIV-XV. fol. 293-340 Theonis commen-

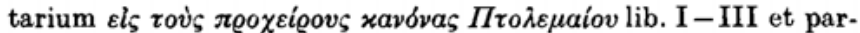

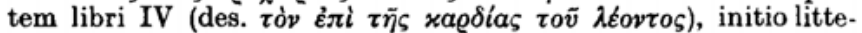
ris deminutis. vol. II quaternionibus $\mathrm{XX}$ unaque ternione $(\lambda \delta$, in quaternione $\lambda \varepsilon$ incipit Theon) constat numeris $\bar{x}-\bar{\mu}$ signatis. in ultimo folio signum est bibliothecae imperialis Parisinae rubro colore allitum. Peyrardus multos locos codicis pulcherrimi et praeter initium finemque optime conservati graphio cerussato notare sustinuit $\left.^{1}\right)$.

Deinde ad codices, quibus ad editionem Theonis restituendam usus sum, et quos I p. IX breviter significavi, transeamus.

Codex igitur Laurentianus XXVIII, 3 membranaceus forma 4 ta pulchra peritaque manu scriptus est saec. X. librarius compendiis plurimis non modo in scholiis, sed etiam in verbis Euclidis utitur $^{2}$ ). continet Elem. I-XV, Optica, Phaenomena, sed male habitus est. nam non modo plurimis locis scriptura antiqua, quae evanuerat, a manu saec. XVI renovata et obscurata est, sed eadem manus, praeterquam quod multas lacunas minores pergameno rupto laceratoque ortas resarcinavit pannis pergameni recentis agglutinatis, totas partes codicis sine dubio tempore et situ ita exesas, ut legi non possent, in pergameno albo nigrisque punctis hic illic distincto supplevit. maiores illae lacunae scripturae antiquae absumpserunt VII 12 p. 119,12 É $\sigma \tau i v$ - IX 15 p. 207,16 $\delta \varepsilon i \xi a l$, quae manu illa recenti, quam $\varphi$ significavi, in XXIII foliis suppleta sunt (exciderunt quaterniones $\vartheta$ et $\imath$ ), et finem inde a XII 3 p. 87, $7 \pi v \varrho a$ (in $H M \Theta$ IV p. 81, 16 desinit quaternio $1 \zeta$, ex quaternione $i \eta$ unum tantum folium exstat). itaque praeter partem extremam Elementorum etiam Optica et Phaenomena manui recenti debentur, nec scimus, quid praeter Elem. I-XIII cod. F ab initio continuerit. nam cum $\varphi$ in scriptura evanida renovanda et in lacunis minoribus explendis plerumque ${ }^{3}$ ) nullo codice alio usa esse videatur - documento sint III p. 163, 7, ubi $\varepsilon i \sigma \iota v \dot{a}$ in ă $a \hat{\eta} \overline{a \eta}$ renovavit, p. 164, 7, ubi $E K$ e pagina opposita litteris $\overline{\chi \varphi v}$ expressis commaculatum est, unde $\varphi$ renovando effecit $\overline{\nu \varphi \chi}$,

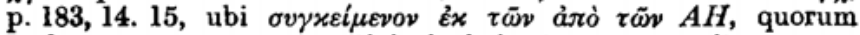
verborum certa exstant vestigia, in àjò $\tau \tilde{\eta}_{\varsigma} \overline{\alpha \eta}$ renovavit $\varphi$, quamquam sic dimidium loci evanidi vacat; ef. etiam IV p. 66, 1 -, contra in duobus illis supplementis maioribus codicem Laurent.

1) In quaternione $a$ folia $7-8$ ante folia $3-6$ transpesita sunt, ita ut folia codicis $16-23$ ita ordinanda sint $18-23,16-17$. etiam error II p. 224, 11 e transpositione foliorum archetypi ortus est. ceterum ex hoc loco et ex errore I p. 118, 23 colligo, archetypum codicis $\mathbf{P}$ litteris uncialibus scriptum fuisse.

2) Correcturae factae sunt et manu 1 et manu 2 satis antiqua.

3) Interdum enim librarius, ubi suo ingenio parum confidebat, hic quoque Laur. XXVIII, 6 usus est, velut IV p. 44, 2, ubi pro io $\eta$ (sic e vestigiis certis in $\mathbf{F}$ fuisse apparet) cum $\mathbf{V}$ et Laur.

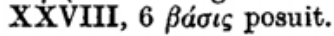




\section{PROLEGOMENA CRITICA}

XXVIII, 6 saec. XIII - XIV descripsit; unde concluditur, cod. F tum demum esse resarcinatum, cum in manus Mediceorum pervenisset. hoc ad demonstrandum $\varphi$ cum Laur. XXVIII, 6 magna ex parte et in libris arithmeticis et in stereometricis, opticis, phaenomenis contuli et in scripturis tantam inveni concordiam, quanta maior cogitari non possit. unum afferam. IV p. 92,15 in $\tau v \gamma \chi \alpha ́ v o v \tau \alpha$ ultima litters in Laur. XXVIII, 6 ita scripta est, ut lineola finalis paullo maior sit; et in $\varphi$ legimus $\tau v$ xávovta (sed $\iota$ erasum). iam codex ille Laur. XXVIII, 6 (membran. forma 4 ta), quem littera $f$ significavi, e cod. Vindob. $V$ descriptus est. nam primum inter libros VII - VIII sicut $\varphi$ scholium illud in textu habet m. 1, quod II p. 237,12sq. edidimus, et quod in $\mathbf{V}$ in spatio vacuo inter libros illos relicto ab eadem manu, qua maxima pars scholiorum illius codicis scripta est $\left(\mathbf{V}^{\mathrm{a}}\right)$, postea insertum est. sed documentum vel certissimum e ratione scholii ad II 13 (infra p. 191) peto. ibi enim in $\mathbf{V}$ scholium 90 primum scriptum erat; deinde cum postea schol. 89 adderetur, locus angustior erat, ita ut prior pars usque ad $\tau \dot{c} \delta$ ic p. 191, 13 ante schol. 90, reliqua post illud poneretur. itaque cum schol. 90 in medio scholio 89 interponeretur, librarius codicis Laur. $\mathbf{f}$ ad sensum non attendens omnia deinceps descripsit; postea demum errorem animadvertit et verba $\pi \circ \iota v \bar{v} \sigma \iota-\dot{\varepsilon} \xi \tilde{\eta} \zeta$ in scholio 89 delevit finique adiunxit. quo quid potest esse clarius? et revera $\mathbf{P}$ semper fere cum $\mathbf{V}$ in scripturis scholisque - nova postea addidit manus recentior $\left(\mathbf{P}^{1}\right)$ - consentit et prorsus eadem opera continet, quae $\mathbf{V}$ (praeter Elementa $\mathrm{I}-\mathrm{XV}$ optica antiqua et phaenomenorum recensionem meliorem, sed in fine mutilam). hine igitur apparet, $\operatorname{cur} \varphi$ et $\mathbf{V}$ tanto opere concordent.

Codex Bodleianus Dorvillianus X, 1 inf. 2, 30 membranaceus est forma 4 ta, Elementorum libros I - XV continens cum scholiis multis. fol. 1 computationes quasdam continet manu recenti (saec. $\mathrm{XV}$, ut videtur), fol. $2-4^{\mathrm{r}}$ quaedam de libro $\mathrm{X}$ Elementorum (v. Append. II nr. 22) manu Arethae, in mg. et in fine fol. $4^{r}$ quaedam mathematica manu rec., quae continuantur fol. $4^{\mathrm{v}}-5^{\mathrm{r}}$. fol. $5^{\mathrm{v}}$ epigramma hoc manu Arethae

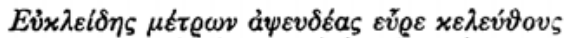

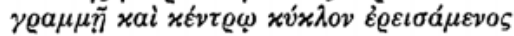

et mathematica quaedam manibus recentioribus. fol. 6-14 pergameni crassi vilisque manu recenti neglegenter scripta continent Elem. I ad I 14 p. 22, 22 ă $\varrho a$ vं $\pi \grave{~(s a e c . ~ X I I I, ~ t i t u l u s ~ e s t ~ E v ̉ x \lambda \varepsilon i \delta o v ~}$ $\sigma \tau o \iota \chi \varepsilon i \omega \nu \bar{a}$ àjò $\sigma v v o v \sigma \iota \tilde{\omega} v \tau o \tilde{v} \Theta \varepsilon \dot{\varepsilon} \omega \nu \circ \varsigma)$. cum fol. $15^{\mathrm{r}}$ litterae multae evanidae sint, apparet, unam quaternionem ${ }^{1}$ ) aliquando periisse, ita ut fol. 15 primum esset (fol. $2-5$ tum alio loco posita fuisse videntur), et postea possessorem aliquem ad lacunam ex.

1) fol. $15^{r}$ numerus quaternionis $\beta$ fuit, sed evanuit, sicut in quaternionibus IX primis. litterae evanidae fol. $15^{r}$ saepe manu recenti renovatae sunt. 


\section{PROLEGOMENA CRITICA}

plendam initium describi iussisse, quod ob genus scripturae novem folia pro octo occupavit. de fonte huius supplementi nihil constat; in fol. 6 librarius scripturam antiquam imitari voluit. fol. $15-118^{r}$ Elem. I 14 -VI fin. manu elegantissima saec. IX, cuius exempla v. apud Wattenbachium et Velsen tab. II et in tabulis 65-66 societatis Palaeographicae Britannicae. in verbis Euclidis compendiis paucissimis, in fine linearum maxime, utitur, accentus spiritusque raro addidit (fecit plerumque manus recentior). fol. $118-120^{\mathrm{r}}$ problemata nonnulla m. 1, sed litteris maiusculis; continuantur man. 1 fol. $120^{\mathrm{v}}-121$, plurimis compendiis. fol. 122 divisiones quasdam (cf. Append. III 1, sub finem) manu Arethae. fol. 123-397 Elem. VII - XV eadem manu, qua fol. 15-118. ultimum folium numero 387 signari debuit; nam errore a pag. 355 ad pag. 366 (pro 356 ) transitur. sed quaternionum numeri, qui antiqui sunt, sed tamen post adscripta scholia positi (nam in quat. $x \delta$ numerus in mg. sinistro, non dextro, ut solet, collocat us est, quia in dextro locus a scholic, occupatus cst), recte procedunt usque ad $\mu \zeta$ (practer $\mu \zeta$, quae ternio est, in $\varepsilon$ et $\iota v$ septem tantum folia sunt, sed vestigia octavi. quod recisum est). fol. $397^{\mathrm{v}}$ duas subscriptiones scripsit Arethas, ques edidit primus Dorvillius ad Charit. p. 229

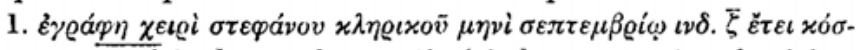
$\mu o v,, 5 \tau \varsigma \zeta$ (eadem verba repetit deinde man. rec.). codex igitur scriptus est anno p. Chr. 888 (v. Wattenbach ad tab. II).

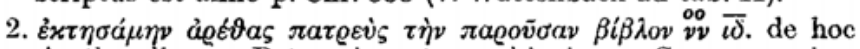
Aretha, diacono Patrensi, postea archicpiscopo Caesareae, eiusque bibliotheca v. E. Maass, Mélanges Graux p. 749sq., ubi etiam breviter indicavit, quid in nostro codice ipse seripserit Arethas ${ }^{1}$ ).

Has subscriptiones sequuntur versus parum elegantes, quos eosdem in $\mathrm{V}$ post finem libri XV et in Laur. XXVIII, 2, 3, 6 invenimus (primus edidit Dorvillius l. c.)

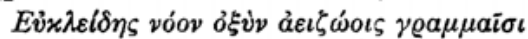

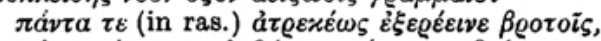

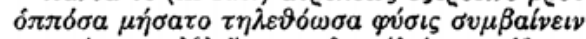

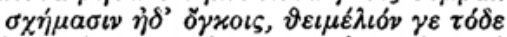

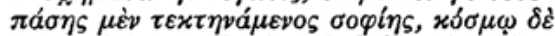

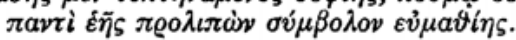

1) Scripsit litteris uncialibus maximam partem scholiorum antiquorum, numeros propositionum librorum posteriorum, subscriptiones librorum I-IX, notas aliquot in figuris, al., praeter ea, quae supra indicavi. idem litteris minusculis errores aliquot librarii correxit. sunt etiam correcturae manus aliquanto recentioris(m.2), quae duobus generibus atramenti utitur (eandem manum esse, apparet ex fol. $179^{\mathrm{v}}$ et $195^{\mathrm{r}}$, ubi in eodem scholio variatur atramentum; recentiorem eam esse manu 1 , ex fol. $180^{\mathrm{v}}$ colligo, ubi initium scholii repetit, quia man. 1 evanuerat). correcturae manus recentis rarissimae sunt. 


\section{PROLEGOMENA CRITICA}

In margine quaedam atramento dedita opera commaculata sunt; fuit fortasse nomen monasterii vel bibliothecae alicuius Italiae; ibi enim Dorvillium hunc codicem praeclarum nactum esse credo; ipse silet. fol. ult. (sine numero) varia, quae legi nequeunt, illevit man. rec.

De codice $\mathbf{V}$ longiore disputatione opus est propter diversitatem scripturae. codex igitur Vindobonensis philos. Gr. 103 apud Lambecium VII p. 391, apud Nesselium XXXI, 13, initio membranaceus est, in fine bombycinus, folia 292 comprehendens forma maxima. continet Elem. I-XV fol. 1-254 ${ }^{\mathrm{r}}$ (in fine libri XV epigramma illud legitur ut in Bodl.; deinde Busbeckius scripsit $\tau \varepsilon \hat{\lambda}$ o૬

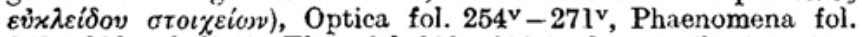
$272-282$, scholia in Elem. fol. 283-292 in fine mutiln. in primo ultimoque foliis Busbeckius seripsit, ,Augerius de Busbecke comparavit Constantinopoli“" (cf. Mosel, Gesch. d. kk. Hofbibl. zu Wien p. 32). fol. 1-183 (quaterniones $a-x \gamma$; numeri quaternionum plerumque et in primo et in ultimo folio notati sunt, sed interdum evanuerunt) sine ullo dubio eadem manu scripta sunt, sed et ductus et atramentum et membrana inaequabilia sunt (atramentum hic illic viride). fol. 184 (inc. III p. 191, $12 \tau \varepsilon \tau \varrho a \gamma \omega \dot{v} \omega \nu v)$ -

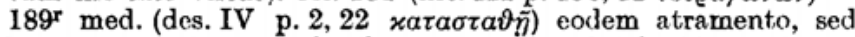
litteris minoribus gracilioribusque. usque ad fol. 190 membrana eadem est (est quaternio $x \delta$; nam 1 folium recisum est), sed fol. $191-202(x \varepsilon)$ membranae sunt crassioris formaeque paullo brevioris, atramento badio. fol. $189^{\mathrm{r}}$ med. $-200^{\mathrm{r}}$ scripta sunt manu celeri et neglegenti, quae initio elegantiam quandam adfectat, sed post paucos versus festinantiae cedit; in fol. 189-190 atramentum furvum est. fol. $200^{\mathrm{v}}-202 \mathrm{manu}$ nitida, sine dubio eadem, quae scripsit fol. 184-189; atramentum badium; versus finem fol. $202^{\mathrm{v}}$ litterae maiores sunt ad paginam explendam (des. IV p. 54, $5 \mathrm{ubi}$ in mg. additur oṽ $\omega \varsigma$ हैं ă $\lambda \lambda \omega$ ). fol. 203-234 eadem manu, membrana, atramento, quibus fol. $1-183$ (quaterniones 4 in primo folio signati $x \zeta x \zeta x \eta x \vartheta$, in ultimo binis numeris $x \zeta-x \zeta, x \eta-x \zeta$, $x \vartheta-x \eta, \lambda-x \vartheta$; fol. $202^{v}$ est $x \zeta-x \varepsilon$, sed fol. $191^{r} x \varepsilon$ tantum ea manu, qua scripti sunt numeri minores, ut videtur, eadem, quae fol. $189-200$ scripsit; numeri maiores in fine quaternionum manui primae debentur). in fol. $235^{\mathrm{r}}$ primi versus septem et dimidius eadem manu, qua proxime antecedentia (des. IV p. 146, $14 B A E$ ); deinde scriptura ita sensim in eam manum transit, quae scripsit fol. 184-189, ut appareat, has duas saltim non differre nisi fortasse calamo; atramentum idem est. deinde in fol. $235^{\mathrm{v}}$ haec manus atramento manente in tertiam velocem cursivamque sensim mutatur, quae eadem maiorem scholiorum partem scripsit $\left(\nabla^{\mathrm{a}}\right)$. hac manu reliqua pars quaternionis $\lambda$ (ita in fol. 242 signata est) scripta est. tum sequitur pars bombycina (inc. IV p. 176, $22 \delta \eta^{\prime}$ ). prima quaternio (fol. 243-250) initio et in fine notatur $\lambda \alpha$ et similiter ceterae ( $\lambda \beta-\lambda \varepsilon$ fol. $251-282$ ), sed in primo folio quaternionum $\lambda \beta, \lambda \gamma, \lambda \delta$ praeterea a m. 1 leguntur numeri $\iota, \iota a, \iota \beta$. in folio 282 desinunt phaenomena in fine mutila. haec omnia (fol. 


\section{PROLEGOMENA CRITICA}

243-282) in charta bombycina tenui laevigataque eadem manu $V^{a}$ scripta sunt, sed ductus atramentumque ob materiae diversitatem aliam speciem prae se ferunt. ultima pars fol. 283-292 (scholia) aliud genus bombycinae crassioris nec laevigatae et propterea alium ductum manus $V^{a}$ habet. quaternio $\lambda_{5}$ (fol. $283-290$ ) numerum in fine habet et praeterea in fol. 284, quia imus margo folii 283 recisus est. e quaternione $\lambda .5$ duo tantum folia exstant. correcturae sunt et primae et secundae manus, scholia multarum manuum, quarum duae codici aequales.

His omnibus perpensis nunc credo, totum codicem eodem fere tempore scriptum esse nec repugnem, si quis eum ab eodem homine scriptum esse contendat; nam quamquam ductus scripturae, si primam et ultimam partem conferas, satis differt, tamen ratio implicata scripturae, pergameni, atramenti non simul mutatorum et manuum inter se transitus in fol. 235 hanc sententiam commendant. hoc saltim constat, totum codicem iam, cum Laur. XXVIII, 6 ex eo describeretur, talem fuisse, qualem nunc habeamus (scholia tamen recentiora et fol. 283-292 in Laur. non sunt; desinit enim in phaenomenis codem loco abruptus, quo Vindob.). quare nunc totum codicem saeculo XII tribuo; neque enim manus prima fol. $1-183$ ad posterius tempus referri posse videtur. iam ante saec. XIII bombycinam in oriente in usu fuisse, quod a palaeographis addubitari video, apparet ex catalogo codicum monasterii cuius-

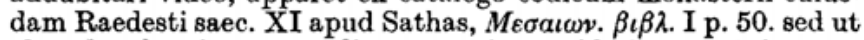
$\mathrm{ab}$ eodem homine partes diversae scriptae videri possunt, ita constat, eas neque eodem tempore eodemve ex antigrapho descriptas esse neque primitus in uno volumine coniunctas fuisse. nam primum numeri $\imath, \iota \alpha, \iota \beta$ in quaternionibus bombycinis $\lambda \beta-\lambda \delta$ (sine dubio in fol. 243 quaternionis $\lambda a$ primo olim fuit $\vartheta$ et in quaternione $\lambda \varepsilon$ numerus $\iota \gamma$; nam fol. 275 in mg. rasura est) ostendunt, has olim membra alius corporis fuisse, quod saltim praeter Optica et Phaenomena libros Elementorum XI-XV continuit, fortasse etiam partem decimi; nam cum fol. $243-254^{\mathrm{r}}$ respondeant pag. $424-450$ ed. Oxoniensis, quaterniones octo ante fol. 243 amissae circiter 120 paginis $^{1}$ ) respondebunt. ita eo fere pervenimus, ubi desinit pars prima (fol. $183=$ III p. 191). fortasse primitus duo volumina erant, quorum alterum praeter quaterniones $\lambda \beta-\lambda \zeta$ etiam $x \delta-\lambda a$ (fol. 184-250) continebat, quae, si cum man. 1 folia $191-202$ numeris $x \varepsilon, x_{\zeta}$ signamus, ipsae illae novem sunt quaterniones. deinde inde, quod phaenomenorum pars extrema deest, et quod in phaenomenis desinit quaternio $\lambda \varepsilon$, concludimus, olim unum praeterea folium adfuisse, quo amisso demum quaterniones $\lambda_{5}$ et $\lambda \zeta$ annexae sint; quarum posterior et ipsa mutilata est. denique fol. 184-202, quae ad lacunam certis finibus circumscrip. tam explendam scripta esse arguit et natura scripturae in fol. 202 extr. et numerus foliorum quaternionis $x \varepsilon$ (quia XII folia erant,

1) Ratione habita figurarum et intervallorum, quorum in libb. XIV - XV magnum numerum habet ed. Oxon.

\section{XXII}




\section{PROLEGOMENA CRITICA}

manus 1 eam numeris $x \varepsilon, x \zeta$ notavit), ex duobus antigraphis et inter se diversis et ab antigrapho reliquae partis codicis discrepantibus descripta sunt. nam fol. $184-189^{\mathrm{r}}$ med. (III p. 191, 12IV p. 2, 22) e codice simili codici P descripta sunt, ut e scripturae consensu apparet. nam non solum discrepantiae maiores, quae in mutationibus Theonis positae sunt, codicum $\mathbf{P V}$ in hac parte com. munes sunt (III p. 191, 17, 20;192, 7; 193, 10;194, 4, 10;195, 2, $8,12,17,19 ; 196,17 ; 197,4,7 ; 198,2,5,19 ; 199,6,23 ; 200,2,4$, 5, 10 et in singulis paginis sequentibus; de lib. XI ci. IV p. 1, 5), sed etiam in erroribus consentiunt (III p. 194, 13; 197, 20;201, 12; 203,$7 ; 204,20 ; 205,4,18 ; 207,18 ; 229,8 ; 231,3 ; 234,7 ; 236,7$ ) et omnino in scripturis omnibus, etiam in minutiis (II1 p. 194, 7 , $10 ; 195,11 ; 198,9 ; 200,7 ; 202,2,17 ; 203,3,10 ; 204,8 ; 205,6.7$; 206,$6 ; 209,20 ; 228,10,12$; cf. p. 194, 14; 197, 3; 206, 8; 207, 8; IV p. 1, 10-14): loci ii, ubi PV discrepant, pauci sunt et omnes eiusmodi, ut in alterutro facillime a librario errari potuerit. neque tamen crediderim, $\mathbf{V}$ ex ipso $\mathbf{P}$ descriptum esse; nam III p. 206, 1 (ubi xai delendum est); 235,$9 ; 236,12.13$ et in litteris figurae III p. 196,$13 ; 202,18 ; 206,14 ; 207,6 ; 229,17 \mathrm{~V}$ cum'Theoninis contra P consentit nec intellegitur, quo modo hic consensus ortus esse posset, si V ex $\mathbf{P}$ descriptus esset; neque enim his locis librario causa erat emendandi (III p. 193, 24; 195, 8; 197, 15; 201, 16; 203 , $8(\tau \tilde{\omega} v) ; 205,7 ; 207,13 ; 209,10 ; 230,3 ; 231,5 ; 232,12 ; 234,6.7$. $8 ; 235,8$ per se parum valent, quia ibi emendatio facile a librario codicis V reperiri poterat; III p. $195,8.9 ; 197,6,9 ; 206,20 ; 235$, 14 iam in $\mathbf{P}$ error correctus est; etiam III p. 210,14 scriptura cod. V e scriptura cod. P orta esse poterat). sed antigraphus cod. $\mathbf{V}$ certe codici $\mathbf{P}$ simillimus fuit, quare in hac particula editio Theonis solis codicibus $\mathbf{F B b}$ nititur, $\mathbf{V}$ ad $\mathbf{P}$ accedit.

Reliqua pars foliorum, de quibus hic loquimur (189r med. - 202) e codice Bononiensi b descripta est. nam IV p. 54, 4 in b $\tau \delta$ et $\tau \tilde{\omega}$ in $\tau \tilde{\omega}$ et $\tau o ̀$ a man. 1 correcta sunt et in mg. additum est oṽ $\tau \omega \varsigma$ $\varepsilon \dot{v} \tilde{a} \lambda \lambda \omega$ ad hanc correcturam respiciens. iam haec ipsa verba etiam in $\mathbf{V}, u b i$ in textu nullum vestigium est correcturae (habet $\tau \tilde{\omega}$ et $\tau \dot{o}$ ut b corr.), in mg. sunt fol. $202^{v}$ eadem manu atramentoque, quibus textus. unde sequitur, $\mathbf{V}$ ex $\mathbf{b}$ descriptum esse (neque enim credo, librarium cod. $\mathbf{V}$ his verbis significare voluisse, se hanc partem ex alio antigrapho sumpsisse; nam etiam fol. $184-189$ aliunde petita sunt tacite). et re vera IV p. $2,23-54,5 \mathrm{~V}$ b saepe consentiunt (v. p. $3,5,25 ; 4,3 ; 5,15.16,25 ; 6,15 ; 7,19 ; 8,20,23$; 10,$1 ; 11,5 ; 12,7 ; 26,14.15 ; 35,1 ; 38,11.12 ; 39,13,14 ; 42,2$; $43,10,16 ; 44,1,2 ; 45,3,14,15,19 ; 46,4 ; 47,14 ; 48,23,25 ; 50$, $4 ; 53,5,13 ; 54,5 ; 192,5.6$; cf. p. 4,$17 ; 5,23 ; 10,22 ; 11,7-12$; $20,7 ; 26,21 ; 39,2 ; 44,6 ; 46,2 ; 50,8,11,14 ; 190,22 ; 191,8)$ et p. $3,2,22 ; 6,14 ; 7,1,2 ; 8,12,20 ; 9,13,21 ; 14,4 ; 15,11 ; 17,4$; $18,5.6,18,23 ; 19,3 ; 21,13 ; 23,4,5,12,14 ; 24,8 ; 25,5 ; 26,18$; 28,$12 ; 32,5,7 ; 34,12 ; 35,10 ; 36,3 ; 37,6,13 ; 38,7$ (nam p. 37 , $13-38,8$ manum $\beta$ secutus est $V$, sicut omnino plerasque correcturas marginales codicis $b$ recepit, etiam manus secundae ut 
p. 52,$2 ; 52,7-53,3) ; 40,5,8 ; 41,1,2,13 ; 45,5,6 ; 51,8 ; 187$, 5,$11 ; 188,3,15,16 ; 189,14,15 ; 190,7,9$ errores codicis b a librario in $\mathbf{V}$ correcti esse possunt. verum tamen alii loci sunt, ubi ap. paret, alio quoque codice usum esse librarium codici B simili; v. imprimis p. 34, 7 (cf. p. 5,$2 ; 9,9 ; 12,17.18 ; 13,25 ; 15,13 ; 16$, $15 ; 25,10 ; 27,15 ; 34,1,5 ; 189,3 ; 190,2,18 ; 191,6,9 ; 192,12$; per se minus valent $\mathrm{p} .13,11 ; 18,2 ; 188,17,18 ; 189,5 ; 190,5,15$, $18 ; 191,1)$.

Itaque si haec omnia animo coniunxerimus, ita rem se habere putaverim. librarius codicis $\mathrm{V}$ primum fol. 1-183 descripsit ex antigrapho, in quo perierant quaterniones duae comprehendentes III p. 191, 12-IV p. 54, 5. sed cum lacunam animadverteret, unam quaternionem eiusdem pergameni seposuit. deinde post lacunam rursus idem exemplar descripsit usque ad finem phaenomenorum. postea aliud antigraphum ad lacunam explendam circumspexit et inde fol. 184-189r sumpsit; sed cum animadverteret, id alius generis esse, rursus ex alio fol. $189^{\mathrm{r}}-202$ descripsit, et cum lacuna maior esset, quam putaverat, XII folia alius pergameni sumere coactus est praeter quaternionem primitus sepositam. primum duo fecit volumina (fol. $1-183$ et 184-282) adiuncta parte bombycina, deinde, postquam perierat fol.ultimum, ex duobus unum additis duabus praeterea quaternionibus; ultimac quaternionis aliquot folia revulsa interiisse, nil mirum est.

Iam ceteros codices Theoninos a me usurpatos describere pergam.

Cod. Bononiensis bibliothecae communalis ${ }^{1}$ ) numeris 18-19 signatus membranaceus est saec. XI ex duobus voluminibus constans forma 4 ta una manu scriptus compendiis multis usa; in $\mathrm{mg}$. scholia habet et manu prima et duabus vel tribus recentioribus scripta, quorum nonnulla recentissima manu Theodori Cabasilae scripta sunt (titulum saepe habent $\vartheta \varepsilon o \delta \omega ́ \rho o v ~ \tau o \tilde{v} x a \beta a \sigma i \lambda a$ vel

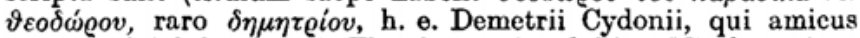
erat Nicolai Cabasilae). is Theodorus, sine dubio a Nicolao oriundus, olim codicem nostrum possidebat; in quaternione $\iota$ scripsit

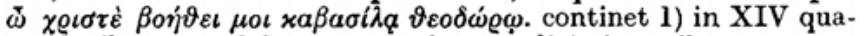
ternionibus $(\alpha-\imath \delta$ in mg. superiore medio), in quibus numerus foliorum sibi non constat, definitiones propositionesque solas (sine demonstrationibus) Elementorum libb.I-XIII et Datorum (XCIII). 2) in quaternionibus legitimis, quarum numeri $\mathrm{m} .1$ in

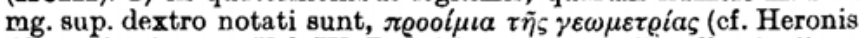
Alexandrini opera. Vol. IV. Definitiones cum variis collectionibus. Ed. J. L. Heiberg. Leipzig 1912, 108-156) et Elementorum libb. I-XIII. in priore volumine sunt quaterniones $a-15$ (des. II p. 191, $4 \delta A$ ), in altero $\iota \zeta-\lambda \varepsilon ; \lambda \varepsilon$ desinit IV p. $182,15 \Gamma \Delta$, ubi in

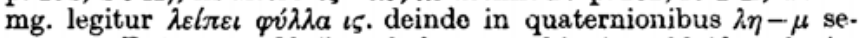

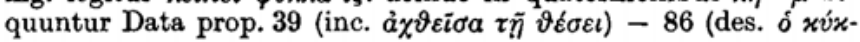

1) Cui donatus est a viro docto s. XVIII A. Magnani soc. Iesu, inter quorum codices erat nr. LXXXII-LXXXIII. 


\section{PROLEGOMENA CRITICA}

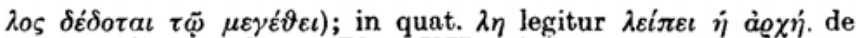
scriptura huius codicis in Elem. XII (et XI extr.) v. IV append. II p. $207 \mathrm{sq}$.

De codd. Parisinis 2344 (q) et 2466 (p), membranaceo utroque saec. XII non multum habeo, quod addam. ille forma maxima est una manu scriptus cum scholiis plurimis complurium manuum;

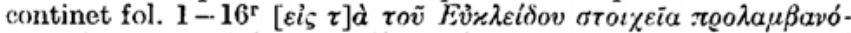

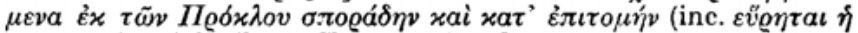

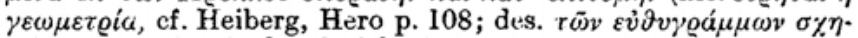

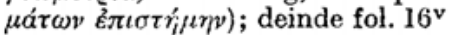

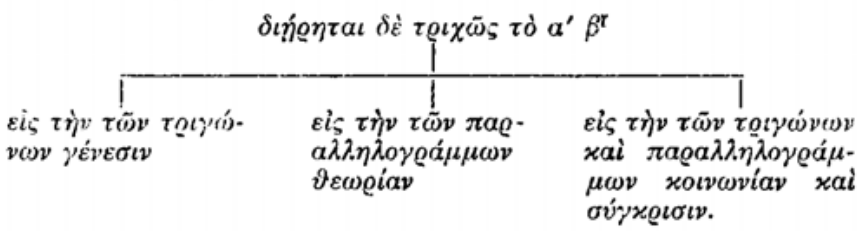

reliqua pars paginae vacat.

Deinde Elem. I - XIII fol. 17 -357 (desunt II p. 186, 3 - 204, 25 ; v. II app. crit. ad locos cit.); ante libb. VII et $\mathrm{X}$ folia aliquot scholiis solis impleta sunt. fol. 358-366 scholia sola continent.

Cod. Paris. p forma 4 ta duabus manibus scriptus est, quarum pulchrior fol. $1-53^{r}$ scripsit in membrana bona, neglegentior fol. $53^{v}-64^{v}$ in eadem membrana, fol. $65-239$ in membrana tenui rugosaque, in qua vestigia sunt scripturae saec. VIII - IX erasae (fuit interpretatio Graeca veteris testamenti, v. Philologus 44, 1885,354 ). continet Elem. I-XIII (post XI, XII, XIII scholia quaedam habet).

Ultimo loco commemorabo palimpsestum Londinensem Musei Britannici Add. 17211 (v. III p. VII et IV p. VII). quinque folia sunt saec. VII - VIII, quae in cod. Syriaco Musei Brit. 687 saec. IX continentur (vol. II fol. 49-53); in singulis paginis binae columnae sunt; dimidium fol. 50 periit (v. Wright, Catalogue of Syriac mss. in the Br. Mus. II p. 548sq.). anno 1847 per Augustum Pacho e conventu Syriaco Mariae Deiparae in deserto Nitriano Aegypti in Museum Britannicum allatus est codex Syriacus 687, cuius vol. I (Add. 17210) notissima illa Iliadis fragmenta continet palimpsesta (v. Catalogue of ancient mss. in the Br. Mus. I p. 6), vol. II praeter nostra fragmenta etiam particulas novi testamenti (fol. 1-48). putant, eum ex codd. CCL iis esse, quos Moses Nisibenus anno 932 monasterio illi donaverit.

Itaque si ad editionem Theonis cognoscendam ex altera parte cod. P, ex altera Theoninos comparaverimus, accidere poterunt casus hi

I. consentiunt

a) aut omnes Theonini cum P; tum scriptura communis, etiam si corrupta vel interpolata est, Theone, h. e. saec. IV, antiquior est. 


\section{PROLEGOMENA CRITICA}

b) aut nonnulli Theonini cum P; tum hi veram scripturam Theonis praebent, reliqui Theonini aberrant; velut PFb contra BV consentiunt IV p. 59, 12; 60, 7. 8; 65, 22; PFV I p. 115,5 al.; PF q IV p. 83, 19; 187, 11; P bq II p. 158, 17; 172, 23. 24; 173, 18; 181,$11 ;$ IV p. 137,$16 ; 138,6 ; 139,14 ; 143,14,23 ; 144,17 ; 145$, $22 ; 146,1-3,3,24 ; 147,2,22 ; 148,25 ; 152,11 ; 155,12 ; 156,14$; $157,13,15,19 ; 158,21 ; 159,5 ; 163,23 ; 164,17,24 ; 174,20,22$; 178,$3 ; 180,22 ; 199,22 ; 200,18 ; 201,8 ; 202,12$.

maxime in eo depravati sunt codd. Theonini, quod alius alibi interpolatus est, quae interpolationes recentiores ope ceterorum Theoninorum cum $\mathbf{P}$ consentientium removeri possunt. quo modo interpolatio sensim luxuriet et bonos quoque codices obrepat, optime iis locis illustratur, ubi verba in nonnullis Theoninis interpolata in bonis codicibus manu recentiore addita sunt, velut I p. 30, $24 ; 56,3 ; 152,19$; II p. 93,$20 ; 126,9 ; 160,19 ; 177,15 ; 180,8$; $181,2-4 ; 183,11 ; 221,3 ; 23$, 8 ; III p. 161, 7; 214, 25; IV p. 148, 25 et definitio analogiae II p. 1, 7; 2, 8; definitio rationis ordinatae II p. 3. 17; propositio tota II append. p. 235 sq. (VII 20); cf. prae. terea IV p. 34, 7; 74, 17. interpolationem sibi propriam habet $\mathrm{F}$ III p. 73.6 ; 129,12 ; IV p. 33,10 al.; B IV p. 51, 8; V III p. 31, 7; 101,$2 ; 102,2 ; 167,17 ; 175,16 ; 176,16 ; 191,9$; IV p. 205, 10 al.; b (unde hic illic interpolatio in alios quoque codices m. 2 irrepsit) III p. 152,$6 ; 159,17 ; 166,18 ; 168,25 ; 195,5 ; 196,25 ; 203,14$, 16 ; $230,3.4$; 231, 9; IV p. 189, 3-11, 14-16 al.

c) aut denique unus solus codex Theoninus contra reliquos cum P consentit; tum quoque hic veram scripturam Theonis habere putandus est, ita ut haec comparatio quasi mensura sit bonitatis fidelitatisque codd. Theoninorum. nullus eorum tam saepe solus cum $\mathbf{P}$ in vera et integra scriptura tuenda consentit quam $\mathbf{F}$, et etiam in rebus minutis mediisque consensus horum duorum codicum magnus cst (v. I p. 34, 4;60, 19, 22;61, 5; 62, 17; 64, 3; 66, $\left.\left.12^{1}\right) ; 67,15 ; 79,17 ; 82,21 ; 87,12 ; 88,9^{2}\right) ; 94,1 ; 102,9 ; 105,18$; $109,5,14 ; 115,14 ; 116,8,9.10 ; 118,11 ; 120,18 ; 124,20 ; 129,8$; 131,$5 ; 137,9 ; 142,14,16 ; 143,15 ; 152,12,14,16 ; 155,18 ; 156$, $20 ; 157,19 ; 160,5 ; 163,10,12 ; 169,17,22 ; 178,4$; II p. 6,$8 ; 12,1$; $15,3.4 ; 74,2 ; 79,9.10 ; 88,4 ; 89,17 ; 216,12$. $13 ; 220,10 ; 221,3$; 222,$10 ; 223,3$; III p. 7,$3 ; 26,4 ; 32,16 ; 73,6 ; 85,16 ; 112,6 ; 144$, $16 ; 145,14,23 ; 147,13 ; 154,26$; IV p. 13,$13 ; 39,20 ; 42,7 ; 61,13$; $\left.78,12^{3}\right) ; 86,22$ et praeter I p. 72,$11 ; 143,4 ; 159,15$, ubi F (m. 1) e correctura scripturam codicis $P$ habet, fortasse etiam I p. 46, 14 ; 134,$1 ; 147,5 ; 166,18 ; 185,4$; IV p. 190, 2; praeterea hi loci ad-

1) Cum his sex locis, ubi $\delta v ́ o$ pro $\delta v \sigma i$ ex optimis codd. restitutum est, ef. I p. $33,12,17 ; 143,4$; IV p. 34,$12 ; 43,16 ; 44,13$;

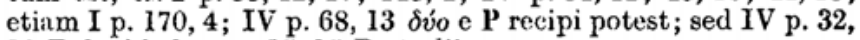
$18 \mathrm{P} \delta v \sigma i$ habet, p. $35,15 \mathrm{P}$ et alii.

2) His tamen locis duobus $\pi 0\llcorner\varepsilon i v$ fortasse etiam in $\mathbf{V}$ fuit.

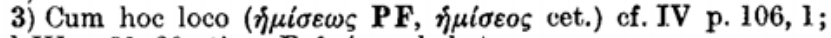

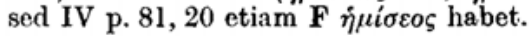




\section{PROLEGOMENA CRITICA}

dantur, quibus $\mathbf{F} \mathbf{m}$. I cum $\mathbf{P}$ congruit, sed a manu posteriore scripturam reliquorum Theoninorum habet: I p. 52, 18. 19; 106, 5, 18 . $19 ; 108,2,20 ; 109,15 ; 115,5 ; 128,8 ; 130,2 ; 133,16 ; 137,11 ; 140$, $19 ; 142,17 ; 145,12 ; 149,20 ; 159,17 ; 162,4,22 ; 166,2,17 ; 172,1$ : II p. 11,$9 ; 39,7 ; 47,17 ; 73,19 ; 105,21 ; 106,1 ; 230,1,18$; III p. 26,$21 ; 95,6$; 105,$7 ; 112,3 ; 189,2$; IV p. 23,2 ; 74, 2 ; minutissima quaedam orthographica pleraque neglexi). haec igitur demonstrunt, $\mathbf{F}$ fidelius quam ceteros recensionem Theonis servasse. unde sequitur, codicem $\mathbf{P}$ in iis partibus, abi $\mathbf{F}$ desit, maius aliquanto pondus habere, etiamsi solus a Theoninis BVbq discrepet. ea de causa scripturam codicis P recepi II p. 127, 21; 148, 7; 155, 12; 156,$18 ; 164,10 ;$ IV p. 90,$3 ; 96,11 ; 100,20 ; 106,16 ; 107,23.24$; 114,$\left.5 ; 117,26 ; 124,13 ; 130,24 ; 141,23 ; 156,8 ; 166,12^{1}\right) ; 172.19$. potueram etiam II p. 131, 12; 132, 12; 150, 10 (cf. 150, 13); 151, $\left.17^{2}\right) ; 190,22 ; 201,14 ; 203,11$; IV p. 138, 4.5 (cf. 138, 8); 143, 8 ; 151, 9, 16; (cf. 149, 17. 18); 182, 26 et in primis IV p. 137, 12; 139,4 (cf. 139, 4 b); dubii sunt II p. 159, 2; 181, 15; cf. 189, 18; IV p. 135,13 et in primis IV p. 113,21, 23; 121, 22; 150, 12, 13; 157,$2 ; 169,24$.

Quanta inter PF necessitudo intercedat, magis etiam ex iis locis perspicitur, quibus iidem errores in utroque codice inveniuntur, velut $I$ p. 5,7 (post. 6 ) ; 44, 1; 129, 13; 133, 6; 140, 17; 166, 11; III p. 105,$5 ; 118,15$; cf. I p. 1,$11 ; 6,4 ; 67,11 ; 131,18.19 ; 147,9$; II p. 92,6; III p. 211, 15. quamquam hic illic consensus fortuitus esse potest, quod I p. 61,$13 ; 143,12$; II p. 64,$10 ;$ III p. 51,$7 ; 52$, $\left.4 ; 59,15 ; 101,19^{3}\right) ; 173,13 ; 197,9$; IV p. 1,6 accidisse puto, tamen negari non potest, nonnullos horum errorum eius modi esse, ut artiorem aliquam necessitudinem codd. PF arguant. hoc ita explicandum esse putaverim, ut dicamus, errores illos iam in eo exemplari recensionis antiquae fuisse, in quod Theon ipse mutationes suas intulerit, ut archetypus editionis suae esset bibliopolaeque describendum traderetur. Theon igitur eos non animadvertit, cuius rei infra alia exempla afferam, et cum erroribus typographicis nostrorum librorum impressorum conferri possunt.

Sed quamquam F Theoninorum longe optimus est, ceteri quoque interdum alius alio loco solus cum $\mathbf{P}$ consentit, solus scripturam Theonis genuinam servavit. hoc in genere haee collegi:

Soli consentiunt PB I p. 95, 4; 151, 15; II p. 11, 10; 169, 17; 189,$15 ; 193,20$. 21; III p. 26,$6 ; 76,8 ; 95,11 ; 129,8 ; 174,6$; 213,6 ; IV p. 3,$22 ; 45,18 ; 50,7 ; 60,6 ; 71,3 ; 74,17 ; 86,5 ; 110$, $15 ; 121,9 ; 124,3 ; 131,14 ; 135,6 ; 141,10 ; 172,1 ; 180,11,12$; 193, 14, 17. loci paueissimi, ubi PB in orroribus conspirant, casui

1) Etiam IV p. 167, 14; 181, 16 codici $P$ obtemperandum fuit.

2) Cf. II p. 125, 20, ubi contra $\mathrm{B} \mathrm{p} \varphi$, quibus addendus $\mathrm{V}$ errore omissus, receptum est $\dot{\alpha} \lambda \lambda$ ' $\dot{\omega}$.

3) Hic $\dot{\eta}$ uncis liberandum est; nam fortuito errore omissum est; cf. III p. 96, 12; 97, 20; 99, 24; 208, 18. cum $\mathbf{F b}$ in mendo fortuito conspirat P III p. 208, 5, cum B p II p. 60, 8. 


\section{PROLEGOMENA CRITICA}

debentur, velut I p. 11, 8; 101, 23; 118, 13; 150, 8; III p. 229, 7; IV p. 85,$13 ; 153,17 ; 191,20$ (III p. 164, 17; 213, 3, 6. 7 nihil valent, quia hic $\mathbf{P}$ postea ad similitudinem codicis $\mathbf{B}$ correctus est). PV (exceptis III p. 191-IV p. 2) I p. 7, 7; 7, 8, 9; 20, 7; 35, 20; $38,12,13 ; 114,15,23 ; 122,16 ; 157,2 ;$ II p. 17,$2 ; 42,2,6 ; 55,21$; $65,3.4 ; 76,2 ; 79,17 ; 81,2,3 ; 83,20 ; 101,7 ; 110,17 ; 169,17$ (ö $\tau \varepsilon$ pro $\tau \varepsilon \delta) ; 210,20 ; 216,4$; III p. 11,$6 ; 12,7 ; 13,5 ; 15,11 ; 16,8$; 19,$16 ; 24,10 ; 36,14,19,22 ; 46,7 ; 63,4 ; 64,3,5 ; 65,8$; 66,$5 ; 83,17 ; 91,11,12 ; 92,20 ; 93,17 ; 96,11 ; 102,3 ; 104,15$; $108,7,22 ; 117,3,11 ; 123,9 ; 132,11,19,20 ; 135,2.3,12 ; 143,4$, $24-144,1 ; 144,4.5 ; 147,10 ; 151,13 ; 152,21 ; 153,24 ; 156,13$; 161,$15 ; 184,17 ; 187,9.10 ; 213,14 ; 218$ app. II ad a ppend. $8 ; 227$, I1 ; IV p. 76,$23 ; 81,4 ; 89,9 ; 96,6 ; 117,12 ; 118,5,11,18 ; 121,7$; 122,$13 ; 123,11,19.20 ; 133,22,24 ; 147,28 ; 171,3 ; 172,13 ; 181$, $18 ; 184,8 ; 200,22 ; 203,19$ (cum $\mathbf{V}$ omnino codici $\mathbf{F}$ affinis sit, nil mirum $\epsilon$ st, $\mathbf{V}$ in partibus, ubi $\mathbf{F}$ deest, saepius quam alibi solum cum $\mathbf{P}$ consentire); cf. etiam I p. 7,$5 ; 128,18$; II p. 108, 24; 111, 1 (de quibus duobus locis cf. tamen p. XLVI) et II p. 86, 23, ubi correctio in $\mathbf{V}$ cum $\mathbf{P}$ congruit. loci pauci, ubi communes errores deprehenduntur, partim incerti sunt (I p. 34, 1; II p. 56, 12;92, 6; IJI p. 127,$6 ; 167,2$ ), partim cius modi, ut casu factum esse possit (I p. 5,$6 ; 25,20 ; 47,1.2 ; 68,12,14.15 ; 112,5$; II p. 92,$13 ; 101$, 2 ; III p. 32,$16 ; 61,4 ; 66,10 ; 142,7 ; 144,1 ; 145,23$; IV p. 10,12 ; $18,11 ; 63,12 ; 66,24 ; 153,2 ; 193,7)$.

Pb I p. 50,$2 ; 52,12$; II p. 133,$9 ; 160,18 ; 191,13 ; 192,8 ; 205$, 13 ; III p. 15, 17; 21, 13;95, 21;97, 3;114, 14;143, 13;146, 1; 153,$2 ; 156,1 ; 1 V$ p. 5,$2 ; 8,10 ; 13,5 ; 14,10 ; 15,13$ (bis); 17, 21; 34,$\left.5 ; 49,14^{1}\right) ; 56,6 ; 57,1,8 ; 61,19 ; 67,4 ; 138,5 ; 139,4 ; 140,7$; 150,$8 ; 164,17 ; 188,5 ; 189,8 ; 201,5 ;$ cf. III p. 120,$21 ; 1 V$ p. 33 , $3 ; 56,6,7 ; 139,9 ; 140,18$. errores communes fortuiti et lenes I p. 23,21 ; III p. 53,$17 ; 93,7$; IV p. 6,$14 ; 17,19 ; 30,19 ; 37,7$; cf. I p. 48,6 .

Pq IV p. 89,$2 ; 91,22 ; 93,10 ; 101,16.17 ; 107,11 ; 108,26 ; 113$, 19 ; 116,$20 ; 124,6,8,12$ (bis); 125,$3 ; 126,2,12 ; 130,5,16,17$; $133,15,20 ; 136,8.9,10,11 ; 147,3 ; 156,5 ; 184,6 ; 186,2 ; 201,19$; 205,$12 ; 206,6$. errores fortuiti communes IV p. 92,$10 ; 148,3$; 158,$3 ; 165,6 ; 185,9$.

Vidimus igitur, regulam supra p. XXVI sub littera c propositam interdum casu eludi, sed hoc idem in regulis $a$ et $b$ fit; nam est, ubi $\mathbf{P}$ aut cum omnibus Theoninis aut cum compluribus in crroribus apertis conspiret. hic illic fieri potest, ut error Theonem fefellerit, sicut supra in F vidimus (p. XXVII), sed sine dubio multo plures casu in utraque codicuin familia sponte orti sunt; et. fere tales sunt, ut sexcenties a quovis librario committantur. a cer-

1) His igitur novem locis $V$, qui in hac parte (IV p. 2-53) e b descriptus est (p. XXIII), scripturam ceterorum Theoninorum habet, sine dubio e codice simili cod. B petitam (v. ibid.); contra IV p. 16,$1 ; 18,5.6$ casu $V$ cum $P$ solus consentit.

\section{XXVIII}




\section{PROLEGOMENA CRITICA}

tissimo exemplo incipiam. II p. 165, 19. 20 enim $\mathbf{P}$ et $\varphi$ (qui e $\mathbf{V}$ descriptus est; $\mathbf{V}$ autem hunc errorem non habet) verba quaedam in mg. habont; cf. IV p. 77, 1 . hoc quoque ad totum genus errorum casu communium illustrandum utile est, quod in termino

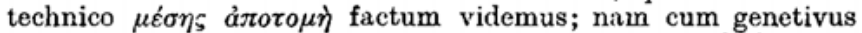
$\mu \varepsilon \sigma \sigma \eta$ in $\mathbf{P}$ saepe servatus sit, etiam ubi in omnibus vel plerisque vel saltim uno et altero 'Theoninorum falso ad àroro $\mu \eta$ accommodatus est, quasi sit media apotome, non mediae (v. III p. 128, 17; $135.18 ; 136,19,23 ; 137,1 ; 139,1 ; 158,23 ; 161,13,19 ; 162,3$; 164,$12 ; 165,1,3 ; 175,4 ; 177,16 ; 189,6,8,9,11,19 ; 190,16$; $196,3,7 ; 197,1,10,15 ; 198,14,16)$, tamen est, ubi idem error etiam in $\mathbf{P}$ irrepserit (III p. 128, 4, 22; 129, 4; 130, 17; 135, 15; 161, 6; sed p. 158, 19 corr. m. 1), interdum etian iis locis, ubi plerique Theorini verum tenent, id quod demonstrat, librarios solus

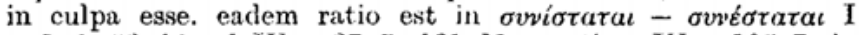
p. 8,$6 ; 92,18$; ef. IV p. 37,$5 ; 161,11$; v. etiam IV p. 105, 7; in litteris figurae I p. 45, 8; II p. 156, 7; IV p. 85, 13, 14, 20, 21, 23; 111, 7, 9, 13. 14; 138, 9; in homoeoteleutis II p. 137, 19; III p. 186,

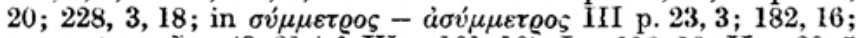
v. praeterea I p. 43, 21 (cf. IV p. 161, 16); I p. 110, 18; II p. 20, 5 (cf. IV p. 42, 8); II p. 37, 3; 39, 10; 41, 17; 104, 23; 114, 12; 115, 15 (cf. p. 154, 16. 17; 186, 3); II p. 176, 20; 215, 3, 14; III p. 10, $13 ; 118,9 ; 140,6 ; 157,7 ; 191,11 ; 212,12 ; 225,2 ; 226,1 ; 232,12$; IV p. 2,$11 ; 87,26 ; 108,22$. conferri potest etiam II p. 53,9 , ubi res ob ordinem verborum parum constantem ccrta est. dubii loci sunt I p. 86, 15; IV p. 3, 8 et in oüt $\omega$ II p. 71,2 ; IV p. 53, 17 (cf. IV p. 59, 10), quia de errore non constat. hic alios quoque locos colligam, ubi scriptura dubia et insolita testimonio codicis $\mathbf{P}$ uniusque et alterius Theoninorum defenditur. III p. 141, $12 \mu i a$

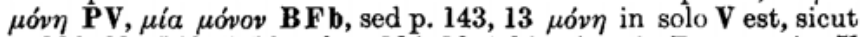
p. 136, 23; 140, 5, 10; cf. p. 134, 13 (ubi $\mu o ́ v o v$ in $\mathbf{P}$ om., $\mu o ́ v \eta \mathbf{V}$ et $\mathbf{F}$, sed corr.) et p. 139, 6 ( $\mu$ ó $\eta \mathbf{V}$ et e corr. F); et p. 135, 15;

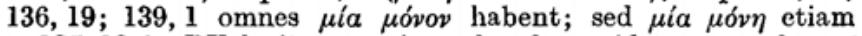

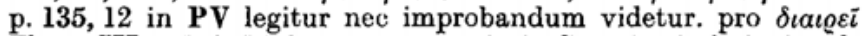
Theon III p. 159, 14 futurum posterioris Graecitatis $\delta \iota \varepsilon \lambda \varepsilon i$ inculcavit et idem fecisse videtur p. 27, 10;91, 7, quamquam his locis

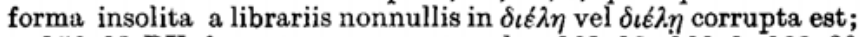

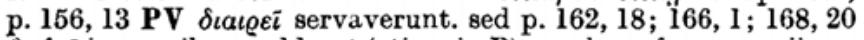
$\delta \iota \varepsilon \lambda \varepsilon i$ in omnibus codd. est (etiam in $\mathbf{P}$ ), nec hanc formam reiicere audeo, cum constet, aequabilitatem sermonis Euclidem minime secutum esse. ${ }^{1}$ ) ordo verborum insolitus $\dot{\eta} A E \mu \dot{\varepsilon} \nu$ III p. 188, 3 tot codd. bonis confirmatur (PBFb), ut eum quamvis dubitans relinquendum putaverim; itaque fortasse II p. 92, $5 \tau \tilde{n} K \Lambda \mu \dot{\nu} \nu$ cum $\mathbf{P F}$

1) Hac de re conferri potest, quod II p. 3, 13. 14; 32, 9 legitur

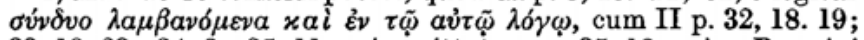
$33,19,23 ; 34,2 ; 35,11$ xai omittatur; p. 35,18 xai e $\mathbf{P}$ recipi poterat. item II p. 138, 15; 144, 1 in omnibus est ov pro solito $\mu \dot{\eta}$; quare II p. 160, 18 ov e $\mathbf{P b}$ recipere non dubitavi. 


\section{PROLEGOMENA CRITICA}

conservandum (contra III p. 189, 15 PV solitum ordinem, BFb illum habent). conferri potest mirus ordo verborum in $i \sigma \eta \mu \dot{\varepsilon} \nu$ IV p. 51, 16, ubi B solus dissentit. at $\tau \dot{o} A Z \delta \dot{\varepsilon}$, quod P III p. 5, 13 habet, non magis recipiendum quam $\dot{\eta} B E \delta \dot{\varepsilon}$ III p. 188, 3 e BFb. cum semper alibi $\pi \circ \lambda \lambda a \pi \lambda a \sigma ı a ́ \zeta \varepsilon \iota v$ legatur, II p. 104, $12 \pi 0 \lambda v$. $\pi \lambda a \sigma \iota a ́ \zeta \varepsilon \iota v$ recipere nolui; sed II p. $175 ; 176$ septies in $\mathbf{P}$ est $\pi \circ \lambda v$ $\pi \lambda a \sigma ı \sigma^{\sigma} a_{\varsigma}$ et quattuor locis (p. 175, 22; 176, 1, 3, 4) eandem formam habet b. tamen crediderim, hic casum aliquem dominari, praesertim cum b non semper cum $\mathbf{P}$ conspiret. similis dubitatio est in forma ov่ $\varepsilon \tau \varepsilon \varrho o \varsigma$, quam III p. 198, 6, 19 praebent $\mathbf{P}$ et nonnulli Theonini, sicut I p. 1,1 ov่vév, et cum de forma per se dubitari nequeat (v. Curtius, Leipziger Studien VI p. 189 sq.), a codicum testimonio discedendum non putavi; sed III p. 188, 16; $204,17,18$ non est, cur e solo $\mathbf{P}$ recipiatur, ne p. 205, $3^{2}$ quidem e PV; nam p. 205, $3^{1}$ in $\mathbf{V}$ solo est, quae inconstantia casum prodit. III p. 151,3 omissio satis dura in P B F defenditur simili loco p. 188, $5-7$; quare potius in ceteris interpolatio quam in his error communis, qui explicari vix possit, statuenda est. in formula tàs $\delta$ vo

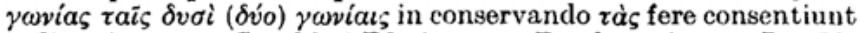
codices (v. tamen I p. 16, 4 Pb p), cum a Proclo omittatur I p. 33, $12 ; 36,4$ et melius absit $\left.{ }^{1}\right)$; $\tau a i{ }_{\zeta}$ vero saepius omittunt (I p. 10, 11; 11, 22 Pp b; p. 17, 1 Pp; p. 33, 12 Proclus, p. 34, 20; 35, 2; 36, 4; 38,$14 ; 173,3$; IV p. 68,21 omnes vel meliores); itaque etsi interdum in bonis codd. (I p. 35, 22; 156,6) vel etiam in omnibus (I p. 15, 23; 16, 4; IV p. 176, 17) legitur, fortasse ubique delendum. angulum significari posse $\hat{\eta} v \pi \dot{d} \tau \tilde{\omega} \nu \Lambda O M$, nunc vix crediderim; itaque III p. 166, $13 \tau \bar{\omega} v$, quod in Pb est, cum B FV deleo; qui error quam promptus fuerit librariis-nocuit $\tau \dot{o}$ vं h. e. $A O \times O M-$, demonstrant loci, quales sunt III p. 169, 5 (B F'b), IV p. 11, 6(P). ne ai $B A \Gamma, A I^{\prime} B$ quidem (omisso vijò) ferri posse credo, etsi I p. 26, 15 in Pp, F m. l, IV p. 190, 9 in $\mathbf{P}$ et $\mathbf{B V}$ m. 1 ita traditum est. hic attigi quaestionem parvam illam quidem, sed ei non prorsus neglegendam, qui aliquando lexicon mathematicum Graecum scripturus est, quae est de formulis mathematicorum Graecorum in rebus mathematicis per litteras notandis. non dico de ordine ipsarum litterarum; neque enim hic locus est promendi, quae de ea re collegi ${ }^{2}$ ), quamquam hoc quoque cum aestimatione codicum coniunctum est. sed duas alias res huc pertinentes tractabo. primum constat, Graecos in producto sive rectangulo significando dicere $\tau \dot{o}$ vं $\tau \dot{o} \tau \tilde{\omega} v A B, B \Gamma$; sed interdum in codd. media littera duarum rectarum communis semel tantum ponitur, ut

1) Non debui tamen IV p. 68, 21 uncis includere (v. app. I ad p. 67,23$)$.

2) Hoc tantum breviter indicabo, mathematicos Graecos in rectis, angulis cet. per litteras significandis id non spectasse, ut eadem res semper iisdem litteris eodem ordine notaretur. litteras eo ordine sumebant, quo in quoque loco ei sese offerebant, qui demonstra. tionem in figura digito sequebatur. 


\section{PROLEGOMENA CRITICA}

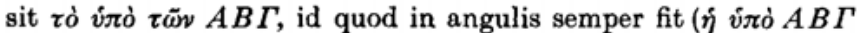
h. e. $\angle A B \Gamma)$. et hoc in codd. optimis tam saepe reperitur, ut vix reiiciendum sit (III p. 30,$5 ; 54,5,14,15 ; 55,18 ; 56,5 ; 57,17$; 60,$16 ; 69,18,19 ; 102,10$ (bis); 104, 12; 107, 11; 108, 15 sq.; 110, 2,$9 ; 111,21 ; 116,19,21$; 184, 9; IV p. 13, 22 sq.; 137, 16sq.; 153, 20 sq.; 158,$10 ; 163,23 ; 200,21$ al.; recipiendum I p. 165, 15; II p. 27,17 (bis); $\mathbf{V}$ saepius quam ceteri formam $A B, B I$ rotinet), quamquam inconstantia codicum in tali re nonnihil offendit (IV p. 200, 21 idem versus utramque formam coniungit). alterum est articulus vel positus vel omissus ante litteras velut $\tau \dot{c}$ dajò $\left[\tau \tilde{\eta}_{S}\right]$

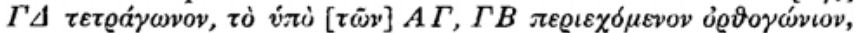

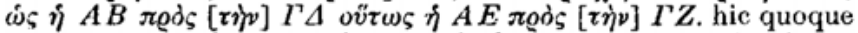
tanta est exemplorum copia, ut articulus non semper retineri possit, quamquam hic: quoque codices parum sibi constant ( $\mathbf{V}$ saepe solus articulum habet, velut IIl p. 116, 6sq.; IV p. 145, 7 sq.; $156,2,15 ; 159,19 ; 199,11 ; 201,2 ; 202,10 ; 206,15$; cum aliis paucis IV p. 153, 20; 156, 14; 157, 8). non raro in $P$ solo deest (I p. 71,$11 ; 75,1 ; 82,17 ; 85,9 ; 90,10,24 ; 145,4 ; 165,20$; II p. 27,$20 ; 28,1 ; 29,19$ sq.; 30,18 , alibi sexcenties), etiam ubi articulus abesse non potest (II p. $75,10,18$ ); sed rursus aliis locis cum $\mathrm{V}$ eum retinet (II p. 47, 22sq.; 48, 22 sq.; 57,$18 ; 58,18$ sq. ; 60, 8sq.; 62,5 sq.; 64, l.0sq.; 81, 2sq.; 96, 4); II p. 173, 18 P cum bq, III p. 27,22 cum b articulum omittit, IV p. 132, 3 solus, p. 132, 5 cum melioribus. in tanta inconstantia rem in medio relinquam et iam ad propositum revertar; restat enim in comparatione codicis $\mathbf{P}$ et Theoninorum casus alter

\section{P et omnes Theonini dissentiunt. tum}

a) si Theonini etiam inter se dissentiunt, $\mathbf{P}$ semper fere verum habet, Theonini varie post Theonem interpolati sunt. exemplo sint I p. 140,$18 ; 185,14$; II p. 21,$16 ; 48,13 ; 129,15 ; 210,2$; III p. 16,$9 ; 56,2.3 ; 61,4 ; 71,15 ; 115,11$ (ubi $\dot{\varepsilon} \sigma \tau \iota v$ delendum); $135,7,9 ; 142,5.6$; 185,$12 ; 229,4$; IV p. $\left.1,6^{1}\right) ; 19,18 ; 34,21 ; 50$, $14 ; 98,8 ; 110,2 ; 141,6 ; 167,21$. etiam II p. 91, 13 scriptura codd. PF eo confirmatur, quod reliqui Theonini non consentiunt; praeterea conferri potest IV p.79,3, ubi $H Z \Lambda \mathbf{P}, \ddot{H} \dot{Z} \Lambda \mathbf{F}, Z H \Lambda \mathbf{V}$, $H A Z \mathbf{B q}$. hic enim scriptura codicis $\mathbf{P}$ et eo confirmatur, quod BV q inter se dissentiunt, et quod $\mathbf{F}$ m. 1 cum $\mathbf{P}$ consentit; nam in $\mathbf{F}$ interdum ordo litterarum cum $\mathbf{P}$ consentiens punctis in vulgarem mutatus est (II p. 99, 5; III p. 109, 23; IV p. 74, 9), nec dubito, quin loci huius generis iis adnumerandi sint, quibus $\mathbf{F}$ a m. 2 ad formam vulgarem redactus est (v. supra p. XXVII). itaque III p. 82, $8 A B$ e $\mathbf{P}$ et $\mathbf{F} \mathrm{m} .1$ recipi potest.

b) si Theonini omnes inter se contra $\mathbf{P}$ conspirant

1) Cf. IV p. 8, 17 et p. 10, 14, ubi fortasse $\tau \tilde{\varphi}$ av่ $\tilde{\varphi}$ e $\mathbf{P}$ recipiendum. 


\section{PROLEGOMENA CRITICA}

a) aut in Theoninis communis error est, qui ad Theonem referri non potest (quamquam hic illic fieri potest, ut eum mendum aliquod latuerit, cum archetypum editionis suae concinnaret). sed interdum sponte in omnibus fortuito ortus est (velut I p. 157, 13; II p. 19, 5 - collato p. 17,21 - ; III p. 140,5; 195, 7; 196, 10; IV p. 111,$8 ; 130,10$ ), saepius autem ad commune nostrorum codicum archetypum referendus est (I p. 34, 11; 134, 9 - ef. p. 133, $11-; 145,1 ; 147,9 ; 154,20$; II p. 15, 20;18,17;24, 8 - cf. p. 23,9 - ; 33, 24 - cf. p. $35,19-; 44,2 ; 63,1 ; 66,5 ; 70,7 ; 76,3$; 83,$22 ; 113,11 ; 127,23 ; 138,16 ; 139,15 ; 156,2 ; 161,18 ; 163,10$. $11 ; 182,6$; III p. 16,$6 ; 22,16 ; 31,11 ; 37,16 ; 50,18 ; 59,5 ; 172,3$; 217,$13 ; 234,12.13 ; 236,7$; IV p. 14, 2 - ubi scriptura cod. P recipienda est - ; 35, 1 - cf. lin. 2 - ; 52, 2. $3 ; 70,11 ; 84,16 ; 91$, $27 ; 93,11 ; 122,5 ; 139,9$; et fortasse etiam II p. 64,$1 ; 67,1 ; 96$, 15 ; 193, 23; III p. 178, 5. 6; IV p. 16, 11.12) ${ }^{1}$ ). hue ii quoque loci pertinent, ubi in litteris figurae erratum est in Theoninis, qui saepe dissensu erroris suspicionem confirmant (v. I p. 136, 15; 137, 12, 17 ; 141, 10; 148, 10; 164, 4sq.; 170, 6sq., 175, 15; II p. 223, 10; III p. 49,$3 ; 55,1 ; 76,9 ; 79,14$ sq.; 80,8 sq. ; IV p. 13, 22. 23; 48, $7 ; 52,7$ sq.; 138, 11; 144, 16; 172, 11 sq.; 178, 15; 195, 13).

$\beta$ ) aut in $\mathbf{P}$ mendum fortuitum, velut I p. 94, 17 ; 95, 9; II p. 48, 6 . 7 ; 197, 5; III p. 109, 18; 112, 23. 24; 155, 8; 161, 4; IV p. 111, $6 ; 160,3 ; 183,21$ et sine dubio etiam I p. 31,13 ; II p. 50,8 ; in litteris I p. 95, 2; 107, 26. minutias colligere supersedeo. ${ }^{2}$ ) hoc tantum addam, saepe aliquid in $\mathbf{P}$ excidisse, plerumque propter

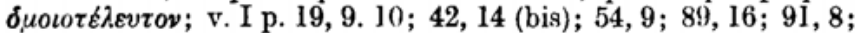
$108,5.6 ; 109,18.19,21.22 ; 167,10-12 ; 177,12 ; 11$ p. 124, 7. 8; 129 , 1. 2; 152, 4. 5; 172,5 , 11. 12; III p. $140,16-19 ; 152,6.7$; 153,$2 ; 163,11.12 ; 171,4-6 ; 181,15 ;$ IV p. 67,$18 ; 82,3.4 ; 131$, $18 ; 134,1 ; 147,20 ; 168,14$; fortasse etiam I p. 35,4 (cf. $36,1.2$ ); 68,$2 ; 89,16.17$; II p. 21,$5 ; 148,15$; III p. 125, 19-21; 178, 22. 23 ; IV p. 184, 19. ${ }^{3}$ )

$\gamma)$ aut $P$ interpolatus est, velut $I$ p. 1,$13 ; 38,5 ; 41,18 ; 43,1,11$; 72 , 19; II p. 33, 17; 111, 24; 143, 6; 179, 2; 214, 7; III p. 120, 13 . 14 ; iV p. 135, 14; 157, 4; 166, 1; his enim locis de interpolatione vix dubitari potest. aliquanto incertiores hi loci sunt II p. 12, 144);

1) Non affero III p. 211, 5. 6, 10, quia totus ille locus in $\mathbf{P}$ postea additus est.

2) De scriptura $\tau \varrho i \tau o v$ I p. 175, 23; 176, 2 dubitari potest; neque enim per se perversa est; v. Archim. III p. IV; sed p. 175, 22

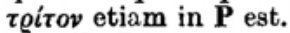

3) De I p. 74, 4-6; 75, 21. 22; 85, 2.3 nunc dubito vellemque verba ibi in $\mathbf{P}$ omissa uncis inclusa esse; nam facillime explicatur, quo modo Theoni in mentem venerit ea addere, nec facile in $\mathbf{P}$ excidere potuerunt.

4) Nam $\mathbf{F}$ fortuito tantum cum $\mathbf{P}$ consentire, ostendit ordo verborum parum sibi constans. 


\section{PROLEGOMENA CRITICA}

113, 2; III p. 53, 11; 61, 2. $3^{1}$ ); et loci haud ita pauci, ubi in $P$ eiusdem modi additamenta deprehendimus, qualia multa Theoni infra

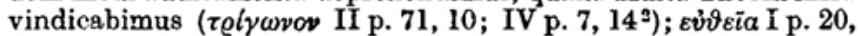

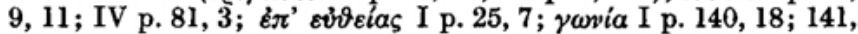
$10 ; 142,5 ; 144,15 ; 148,11$; II p. 51, 6; 101, 20; $\varepsilon \varepsilon \tau \varrho \alpha ́ \gamma \omega \nu \nu \nu$

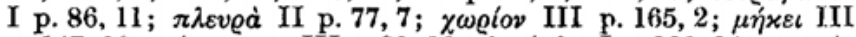

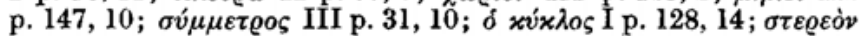

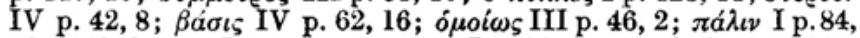

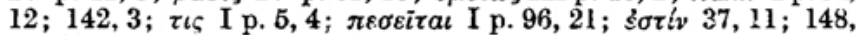
12 ; II p. 53,$10 ; 81,6$; III p. 43,$8 ; 51,11 ; 54,3.5 ; 61,8 ; 68,4$; 71,$15 ; 72,13 ; 82,24 ; 95,18 ; 97,10 ; 99,2 ; 106,25 ; 130,10 ; 142$, $18 ; 146,17 ; 164,7 ; 186,23 ; 217,17 ;$ cioi III p. 42, 18). sed etsi de uno et altero loco antea aliter censui et etiam nunc dubito ${ }^{3}$ ), tamen, si summam spectes, nunc quidern credo, haec omnia interpolationibus codicis $\mathbf{P}$ tribuenda esse, cum reputo, quam proclive

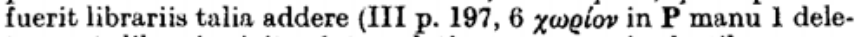
tum est; librarius igitur interpolationem, quae ei sub stilum venerat, ipse temperi animadvertit); nec intellego, aut cur Theon haec delere voluerit, aut quo modolibrarius archetypi nostrorum codicum Theoninorum in omittendo, quae ne minimam quidem offensionem haberent, immo saepe orationem planiorem redderent, tam saepe peccare potuerit. multo facilius intellegitur, cur librarius codicis $\mathbf{P}$ haec addiderit.

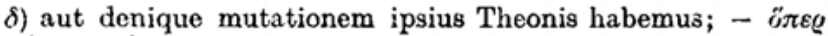

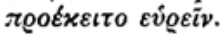

Quas mutationes Theonis cum perlustraverimus, inveniemus, ut par est, eum in Elementis edendis nihil fere mutasse, nisi ubi causam aliquam, interdum futilem illam quidem, et quae nobis non probetur, sed aliquam tamen, qualiscumque esset, sibi habere visus esset. et quamquam de meritis eius non nimis honorifice iudico, tamen a me impetrare non possum, ut discrepantias levissimas, ubi et scriptura Theoninorum et ea, quam $\mathbf{P}$ praebet, per se bona est et probabilis, Theoni tribuam. quare is locis, ubi ne minima quidem excogitari potest causa, cur Theon, si scripturam codicis $\mathbf{P}$ ante oculos haberet, eam mutaverit, non statim codici $\mathbf{P}$ principatus debetur, nisi constantia quaedam in discrepantiis ostendit,

1) Saltim Theonis nomen in notis criticis tam confidenter ponere non debui, id quod etiam in nonnullis locorum sequentium valet.

2) I p. 56, $16 \tau \rho l \gamma \omega v o v$ aegre caremus; nam sicut certum est,

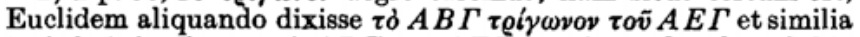

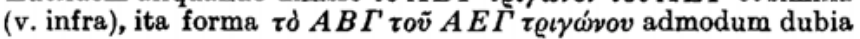
est.

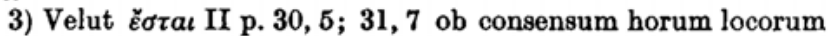
fortasse recipiendum fuit. rursus constantiae cod. $\mathbf{P}$ in $\delta \dot{\eta}$ post $\delta \mu o i \omega s$ addendo II p. 12, 12; 14, 24 non tantum tribuo, ut $\delta \eta$, quod vix Graecum est, recipiam. 


\section{PROLEGOMENA CRITICA}

eas non in casu aliquo, sed in voluntate positas esse. in iis, quae àdıáqo@a sunt, eandem legem, quae in rebus criticis omnino regnat, sequendam puto, scilicet ut vetustati fontium ius suum servetur. et vetustiores esse fontes editionis Theoninae negari non potest. nam non solum palimpsestus Londinensis, qui cum ceteris codd. nostris artissima cognatione coniunctus est, duobus saeculis minimum antiquior est codice $\mathbf{P}$, sed etiam reliqui Tr eonini eo modo inter se cohaerent, ut ab archetypo communi, quod inter eos et ipsam editionem Theonis interesse supra p. XXXII demonstravi, compluribus membris mediis dirempti esse iudicandi sint; quare cum ipsi codicem $\mathbf{P}$ aetate vel aequent vel superent, archetypum illud longe eum superat. itaque eo magis ratio habenda est mutationum, quibus librarios interpolatoresque (nam horum quoque manus supra p. XXXII deprehendimus)editionem antiquam, cuius testem solum cod. $\mathbf{P}$ habemus, inquinavisse veri simile est. ${ }^{1}$ ) negari non potest, rationem, quam exposui, hoc habere incommodum, quod judicium de editione Theonis non semper causis certis confirmari possit, sed ex opinione probabilitatis interdum pendeat, quo fit, ut multa arbitrio relinquantur. haec qui consideraverit, non mirabitur, me de multis locis iudicium, quod in ipso opere concepissem, eo absoluto, cum omnia clariora, collecta, ad perlustrandum promptiora essent, paullatim mutasse. sed hoc in. commodum eo minuitur, quod ii loci, de quibus dubitari potest, plerumque non magnum momentum habent, et quod de editione Theonis omnino probabilitate sola iudicamus, quoniam veri simile est, multas scripturas, quas nunc e nostris codd. Theoni tribuimus, pluribus codd. Theoninis collatis Theone posteriores inventum iri.

Earum discrepantiarum, quas ádıáqo@a vocavi, et in quibus Theoninos, non $\mathbf{P}$, sequendos esse existimavi, quia Theoni imputari non possunt, haec sunt exempla potiora I p. 102, 19; 148, 10

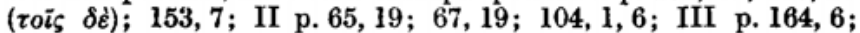
176,$\left.13 ; 182,2,3^{2}\right)$; in litteris I p. $59,4.5$; II p. 14, 14, 17; 70, 8; $226,4,5$; III p. 21, 16; 181, 14; IV p. 11, 10 (III p. 92,3;IV p. 22, 1 fortasse scripturam codicis P recipere non debui); minutias omisi. in ordine verborum maior est numerus locorum: I p. 79, 2; 97, 17; 112,$\left.6 ; 117,16 ; 128,18 ; 130,10 ; 157,16 ; 184,5,22^{3}\right)$; II p. 17, 5; 22,$18 ; 216,8$; III p. 39,$5 ; 52,5 ; 108,9$; IV p. 18,$15 ; 38,5.6$; 69,15 . scripturam codicis $P$ dubitanter recepi his locis, qui fortasse melius huic classi adnumerandi erant: III p. $26,13.14 ; 99$, $10 ; 121,12.13 ; 130,7.8 ; 131,14$ (ubi tamen propter $\mathbf{F}$ magis ad

1) Hanc sententiam cum in studiis Euclideis p. 180 adum. brassem, quaedam oblocutus est $\mathrm{H}$. Weissenborn, Philol. Anzeiger XV p. 39 ; sed mihi non persuasit. quare sententiam meam pluribus explicandam duxi.

2) Nam consensum codicis $\mathbf{V}$ fortuitum esse, ostendit ipsa incon. stantia.

3) Hoc loco fieri pctest, ut ordinem Theon mutaverit ob sententiam relativam. 


\section{PROLEGOMENA CRITICA}

partes codicis $P$ inclino); 177, 2; 217, 18; IV p. 72, 13; 74, 1, 2, 3. in rebus orthographicis nullum est momentum codicum. quare II p. 107, 4 aiki pro à $\varepsilon i$ Theoni tribuere non debui. qui ineonstantiam codicum hac in re cognoscere velit, comparet IV p. 81, 24; 83, 12; $84,1,12 ; 93,12 ; 106,25 ; 110,14 ; 119,1,4,14,15,19 ; 135,23$;

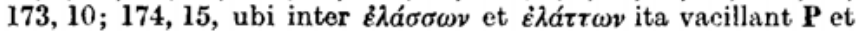
Theonini, ut unam formam semper restituere non tanti esse putaverim; hoc tantum curavi, ne duae formae nimis inter se eodem loco permiscerentur.

In III p. 191, 12 - IV p. 2, 22 eadem prorsus ratio est, nisi quod ibi praeter $\mathbf{P}$ etiam $\mathbf{V}$ editionem antiquam praebet; itaque editio Theonis e PV prorsus eodem modo iudicanda est, quo alibi e $\mathbf{P}$ solo. inter Theoninos hic quoque $\mathbf{F}$ ad recensionem antiquam (PV) proxime aecedit (III p. 192, 17; 198, 2-4; 229, 4; 231, 17; 233,19 ); cum $V$ solo in rebus levissimis casu consentit III p. 195, $10 ; 197,21 ; 198,14,19 ; 203,7 ; 207,8 ; 210,13.14 ; 231,14 ; 232$, 17. item casu factum est, ut consentiant BV III p. 198, 2 et (in numeris propositionum) p. 202, 5; 205, 8; 208, 7; V b III p. 207, 2; 210,$2 ; 231,1 ; 235,16$; VBb III p. 200, 11; VFb III p. 203, 4; 210, 8 (rursus III p. 208, 9 fieri potest, ut $\tau \varepsilon$ in $\mathbf{P b}$ sponte interpolatum sit). ${ }^{1}$ ) de erroribus certis codicum PV v. supra p. XXIII. de III p. 236, 12 et de $\dot{\varepsilon} \sigma \tau i v$ in Theoninis omisso p. 198, 20;206, 5; 234, 20 dubitare licet; cf. etiam p. 235, 7. cum $\mathbf{P}$ aliquanto melior sit quam V (hic proprios errores habet III p. 192, 8; 195, 13, 15; $196,5,20 ; 197,1.2 ; 198,5,6,18 ; 199,19 ; 200,3,5 ; 201,14.15$, $15 ; 202,11,14 ; 203,1,9 ; 204,12 ; 206,12 ; 207,17,19 ; 208,24$; $209,3,9 ; 231,7 ; 232,2,12 ; 233,12 ; 235,19 ; 236,8$, plerumque tamen leves et iam a manu 1 correctos; ả $\delta$ ı́́qoo $\alpha$ sunt III p. 196, $21 ; 198,6 ; 203,17 ; 206,16$ ), et cum III p. 236,4 V manifesto interpolatus sit, quaeritur, num alicubi $\mathbf{V}$ et Theonini casu communem interpolationem habeant. hoc factum esse credo III p. 194, 1; 204,14 , et p. 233, 3 quidem interpolatio in avंzoĭs prope certa est (etiam p. 234, 1 av่zoüs in PV fortasse interpolatum est).

Vidimus (p. XVII), librarium codicis $\mathbf{P}$ sive potius archetypi eius recensionem antiquam dare voluisse. itaque si scribendi erro. res interpolationesque removerimus, de integritate scripturae manus primae non est cur dubitemus, ne ibi quidem, ubi correctura a manu prima statim facta est (velut I p. 12, 2; 27, 9. 10; 56, 18; 61,12 . 13; 64, 12; 71, 11. 12; 109, 24; 113, 6. 7, 23;122,11. $12 ; 123,15-17 ; 162,2 ;$ II p. 11,$11 ; 15,24.25 ; 27,2 ; 79,13 ; 173$, 12. $13 ; 174,4.5,10$; III p. 115,$11 ; 183,1.2,21 ; 189,14.15 ; 207$, 16). sed multis locis manus 1 postea alio atramento correcturas fecit, maxime addendo, quae in textu desiderantur; et ex ratione scholiorum libri primi constat, hanc manum primam posteriorem

1) E correctura V consentit cum F III p. 206, 12, cum B p. 207, 14, cum b p. 204, 17, cum B b p. 198, 6, 18; 232, 7, cum Theoninis omnibus p. 192,$7 ; 198,2,22 ; 200,2,7 ; 204,2 ; 204,20-205,1$; 206,$13 ; 208,4 ; 233$, 15. 16. III p. $200,1,2$ de collatione dubito. 


\section{PROLEGOMENA CRITICA}

novis fontibus usam esse (nam ea scripta sunt scholia libri I, quae $\mathbf{P}$ solus habet). itaque videndum, ne in supplementis illis codice Theonino usa sit. et quamquam saepe emendationes huius manus aperte verae sunt et certa menda tollunt, sive eas ex archetypo ipso codicis $\mathbf{P}$ sive e libro aliquo Theonino sumpsit (v. I p. 39, 7; $58,1.2 ; 59,20.21$; IV p. $7,7.8 ; 102,8 ; 137,7.8 ; 141,15.16$; $188,20.21 ; 201,15.16,17.18$ ), tamen saepius etiam talia supplet, quae et supervacua sunt et difficulter errore librarii non oscitantis in $\mathbf{P}$ excidere poterant; eius modi additamenta in primis sunt demonstrationes alterae X 1,6,9 (III append. 1-3; nam etiam III append. 2 a manu 1 posteriore in mg. addita est); haec cum sine dubio e cod. Theonino interpolata sint, idem factum esse potest I p. 27,2 , ef. 59,$2 ; 36,1.2 ; 42,22.23$; III p. 84, 3, 4; 104,

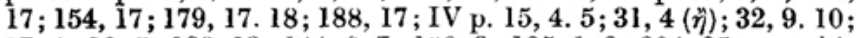
57,$4 ; 80,5 ; 139,13 ; 144,6.7 ; 160,3 ; 185,1.2 ; 204,25 ;$ nam his locis omnibus manus 1 postea ${ }^{1}$ ) supplementa addidit, quae nunc damno, quamquam hic illic additamenta illa sine suspicionis nota practermisi. cum toto hoc grenere conferri potest etiam correctio IV p. 16, 17 in $\mathbf{P}$ mg. a manu prima postea adscripta.

Manus recentioris, quae et ipsa in $\mathbf{P}$ quaedam correxit, nulla prorsus auctoritas est; nam apertissime scripturas Theoninorum invexit, velut in VI 33 (v. praeterea I p. 38,$6 ; 79,1 ; 110,16 ; 130,6$; II p. 9,$2 ; 79,13 ; 113,20 ; 142,8 ; 148,9$; III p. 5,$19 ; 68,15$; 85,3 sq.; 87,3 sq.), easque e libro Theonino non optimo sumpsit (v. I p. 131, 5; II p. 117, 12; 129, 18).

Iam restat, ut de cognatione codd. Theoninorum a me usurpatorum - nam de reliquis Theoninis a me hic illic inspectis alio loco agam - paucis exponamus. quamquam constat, codd. FBV b p q omnes a communi archetypo, quod ipsa editione Theonis recentius est, derivatos esse (v. supra p. XXXII), tamen ex iis locis, quos p. XXVII sq. attuli, apparet, eos inter se alium ex alio descriptum non esse. qui enim, si ita esset, fieri posset, ut singuli multis locis soli cum $\mathbf{P}$ in scriptura genuina consentirent? de solo $\mathbf{p}$ ibi non dixi; quare hic ab eo incipiam. p igitur eum $\mathbf{B}$ artissima cognatione coniunctum esse, ostendunt scripturae horum codicum

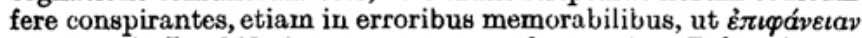

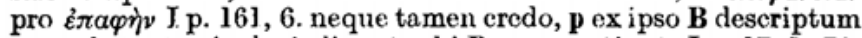
esse. obstant enim loci aliquot, ubi Pp consentiunt: I p. 17, 1; 76, $8.9 ; 98,23 ; 106,13 ; 161,12.13$; II p. 103, 2; per se minus valent I p. 5,$6 ; 27,5 ; 77,13 ; 102,19 ;$ II p. 114, 12; 147, 12 et in litte-

1) Alia res est, ubi man. $\mathbf{l}$ in $\mathbf{P}$ statim quaedam mg. addidit, quae interpolationem sapiunt, velut I p. 9, 22; 21, 11; XII 16

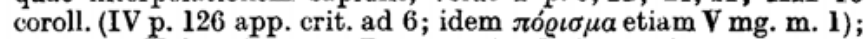
I p. 9, $22 \widehat{V}$ fortuito cum $\mathbf{P}$ consentit. de interpolationibus quibusdam, quas Theonini in verbis Euclidis, $\mathbf{P}$ in mg. tantum habet, postea videbimus. - II p. 220,4 oi $A, \Gamma$ fortasse postea add. a manu l. IV p. 203, 14 additamentum a man. 1 postea factum fortasse omitti potest, praesertim cum etiam in $\mathbf{V}$ in mg. sit. 
rarum ordine consensus I p. 70,$21 ; 72,11 ; 75,22 ; 79,11,14 ; 82$, $15 ; 92,10 ; 109,1 ; 112,9 ; 113,23 ; 114,10$; II p. 29, 22; 146, 7 . errores communes plerumque fortuitos adnotavi hos: I p. 35, 15; 70,$8 ; 114,15.16 ; 167,18$; II p. 107, 6; 148, 14 et praeterea I p. 78, 18, ubi consensum mero casui deberi apparet ex p. 81, 1. etiam I p. 77, 13 nunc credo, ovv fortuito in ambobus interpolatum esse. I p. 3, 9 Pbp soli consentiunt in vero ordine verborum. rursus scholium ad VII 39 (II append. p. 237) initio libri VIII in textum receptum coniunctionem quandam cum $\mathbf{V}$ significat.

Ceterum stemma codicum F BV b q dari non potest; nam et in consensu et in dissensu tanta est horum codicum inconstantia, ut appareat, eos eodem fere gradu ab archetypo distare. huc accedit, quod codicum familiae correcturis inter se permix tae sunt. velut in $\mathbf{V}$, cuius librarium in quadam saltim parte codice codici $\mathbf{P}$ simili usum esse ostendimus, etiam alibi vestigia sunt, quae eo ducunt, ut putemus, eum ex hoc codice correctum esse. nam additamentum in IX 19 (v. vol. V pars 2) vix ex alio codice petitum esse potest; quare idem fons est additamenti secundi in IX 19 (v. ibid.), quod etiam ex F sumptum esse potuit. contra interpolatio mani. festa in IX 30 (vol. V pars 2) aliunde est petita. haec tria additamenta deinde in $\mathbf{f}$ in textum recepta sunt. eodem refero, quod III p. 69,7 ; IV p. 110, 23 aperta menda codicis $P$ solius in $V$ illata sunt correcturis; cf. I p. 32, 4; III p. 38, 12; IV p. 161, 4. sed librarius in codice $\mathbf{V}$ corrigendo etiam alium codicem usurpavit; nam in additamentis illis IX 19 et 30 adscriptum est manu $V^{a} \dot{\varepsilon} v \tau \tilde{\omega} \beta \iota \beta \lambda \ell \omega$

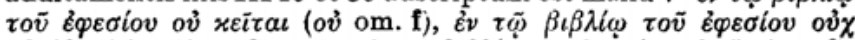

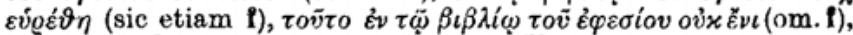
quae verba librarius postea adiecit, cum $\mathbf{V}$ correctum cum novo exemplari conferret. quis fuerit ille Ephesius saeculi XII divinare non possumus. commemoratur etiam ad X 23 coroll. in V (III

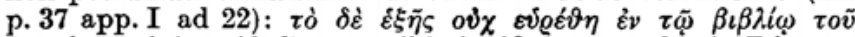
$\dot{\varepsilon} \varphi \varepsilon \sigma i o v$ xai $\dot{\varepsilon} \pi \alpha \tau \eta \dot{\vartheta} \eta^{1}$ ); quae ibi significantur verba, in $\mathbf{P}$ leguntur, sed alio loco, in F B b omittuntur, nisi quod in $\mathbf{B}$ addita sunt m. 2. correcturae codicis $V$ modo cum B consentiunt (I p. 4l, 21;

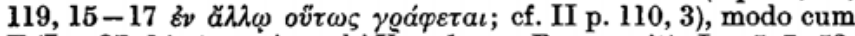
F (I p. 37, 14 et saepius, ubi $V$ m. 1 cum $P$ congruit); I p. 5, 7; 52, 18. 19; 155, 18; II p. 28, 1 V m. 2 cum $P F$ consentit. inter $V$ et $q$ cognationem aliquam esse, in scholiis certe, apparet ex scholio ad

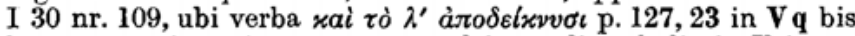
leguntur, cuius rei causa est, quod in medio scholio in $\mathbf{V}$ inter-

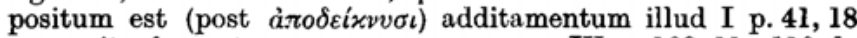
app. crit. cf. praeterea errores communes IV p. 109, 11; 110,1; 137,$11 ; 148,23 ; 206,7$. de $\mathbf{F}$ hoc memorabile videtur, interdum manum 1 interpolationes Theoninorum deteriorum invexisse vi. deri (I p. 46, 14; III p. 62, 17; 149, 24); sed ob paucitatem locorum res incerta est. F m. 2 interdum memorabiliter cum b congruit (III p. 57, 6; 88, 19; IV p. 21, 13; cf. p. 19, 2, 18 et p. 1, 5); rursus

1) Nescio, an $\pi a \tau \varepsilon i \nu$ significare possit: cum contemptu reiicere. 


\section{PROLEGOMENA CRITICA}

in b III p. 57, 6; IV p. 19, 2, 18 scriptura codicis $\mathbf{F}$ in mg. est ( $\gamma o$. .) a m. 1. B m. 2 persaepe corrigendo scripturas codicis $\mathbf{P}$ inculcat, velut III p. 122, 12;124, 2;136, 17; 147, 10; 173, 6; 178, 5. 6; 181,$18 ; 184,15 ; 189,8 ; 234,1$; IV p. 84,16 et falso 206,13 ; cum PV consentit III p. 235,3 ; IV p. $1,10-14 ; 18,5.6 ; 123,19.20$ et falso p. 161, 4; B m. rec. et F I p. 136, 4. errores notabiliores codicum B q communes sunt IV p. 94, 6. 7; 109, 24; 116, 11; 119, 13, 23. 24; 123, 19. 20, codicum Bb II p. 203, 15; 204, 17. 18; 205, 23; III p. 63, 4 alibi. in b interdum scripturae codicis $q$ correctura restitutae sunt, velut IV p. 172, 17; 174, 25; 179, 14; 181, 5 (bq consentiunt IV p. 179,$21 ; 180,7,16)$. IV p. 59,9 Fb corollarium omittunt soli (addidit in b manus prima).

Denique palimpsestus $\mathbf{L}$ semper fere cum B consentit, etiam in mendis apertis (v. Philologus 44, 1885, 366), velut III p. 26, 16, $18 ; 52,5,8,11 ; 53,12,16,17 ; 54,5 ; 136,6,11 ; 137,7,12 ; 138,2$; $204,5,7 ; 205,21$; IV p. $165,5,24$. 25. discrepantiae paucae et fere levissimae sunt: III p. $25,16,19 ; 26,6 ; 52,19 ; 53,9$ (bis); 54,$1 ; 137,20-22 ; 138,1,2,4,7 ; 204,1$ (bis), 4, 6, 9; 205, 1, 5, 10, 19, 23; IV p. 164, 21, 24; 165, 1, 2, 3, 5, 10 in solo $v$ positae sunt, quod in $\mathbf{L}$ semper fere in $\dot{\varepsilon} \sigma \tau \iota v$ et eílv additur; paullo maiores sunt III p. 26, 18; 51, 23; $53,13,16 ; 54,2,8,9 ; 137,11 ; 138,7 ; 204$, I, 14, 19; 205, 8; IV p. 164, 11, 17. errores in solo $L$ reperiuntur III p. $52,9,12 ; 136,18 ; 137,5 ; 204,14 ; 205,9 ;$ IV p. 164, 1, 5, 16, $27.18 ; 165,12$.

Horum igitur codicum ope nobis licet codicem $\mathbf{P}$ comparantibus de editione Theonis veri similiter iudicare, sed verisimilitudine quadam contenti esse cogimur. nam primum saepe difficile est diiudicatu, utrum scriptura codicis $\mathbf{P}$ re vera genuina sit, reliquorum a Theone illata, an hi Euclidis manum praebeant, $\mathbf{P}$ errorem. deinde fieri potest, ut inter codices Theoninos, quos conferre non potui, unus et alter sit, qui alicubi ad $\mathbf{P}$ propius accedat quam mei, id quod iudicium de scriptura Theonis mutaret. omnino credibile est, editionem Theonis minus a $\mathbf{P}$ discrepasse, quam Theonini mei ostendant, quoniam constat, eos communi archetypo ab illa diremptos esse. sed cum et antiquissimi sint et tam inter se dissimiles, ut commune illud archetypum, quod ex eorum scripturis restitui potest, longo temporis intervallo a Theone distare non possit, sperare possumus, nos iam nostrorum codicum auctoritate confisos in universum recte de mutationibus Theonis iudicium facere posse, etiamsi codices postea collati scripturam aliquot locorum mutaturi sint.

Iam igitur ad mutationes Theonis colligendas editionemque eius restituendam transeamus. 


\section{PROLEGOMENA CRITICA}

\section{Cap. II \\ De recensione Theonis}

Primum igitur Theon, ubi in codicibus suis aliquid invenit, quod contra mathematicam peccat, errorem, ut editorem decet, emendare conatus est. lacunas tamen incuriasque, quae in libris stereometricis maxime occurrunt, non animadvertit. hoe eius studio rarissime tantum opus erat, quia rarissime eiusmodi errores velab Euclide vel a librariis commisai sunt; reconditiores enim, ut dixi, non intellexit. huius generis conatus Theonis his locis inveni:

VI 19 Euclides corollarium addidit vix satis ipsa propositione

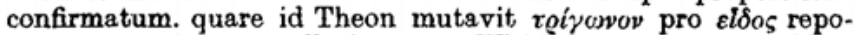
nens et genuinum corollarium post VI 20 demonstratione addita colloca vit; cf. II p. 72 app. I ad 2-5.

IX 19 recte intellexit, conclusionem p. 211,10 sq. falsam esse, sive Euclides ipse, quod magis crediderim, sive librarii erraverunt (si librarii in culpa sunt, totum demonstrationis tenorem mutaverunt, quod parum credibile est, si Euclides verum dederat). quare totam demonstrationem immutavit, sed parum feliciter; neque enim eum casum pertractat, ubi $A, B, \Gamma$ deinceps proportionales non sunt; ea ipsa de causa in $\pi \rho o \tau a ́ \sigma \varepsilon t$ scripsit $\varepsilon l$ pro $\pi \delta ́ \tau \varepsilon$ (p. 210, 22, 25); cf. II p. 211 app. I ad 11-13.

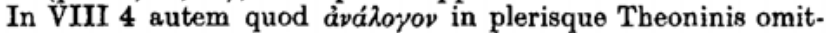
titur, vix emendationi Theonis debetur; nam p. 154, 6; 155, 4 in $\mathbf{V}$ additum est a manu 1 , in $q$ in textu est. et fortasse usus insolitus verborum $\dot{\varepsilon} \xi \tilde{\eta} \zeta \dot{\alpha} v a ́ \lambda o \gamma o v$ defendi potest (II p. 154 app. I ad 6). ${ }^{1}$ )

IX 11 corollarium prorsus necessarium omisit, credo, quia ob

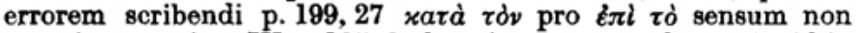
perspiceret. etiam IV p.185, 8 ob scripturam mendosam (quid in P fuerit, nescimus, sed rasura ipsa ostendit, aliquid peccatum fuisse) coniecturam violentam nec verisimilem ( $\dot{\alpha} \lambda \lambda^{\prime}$ o $\delta \dot{\delta} \delta \dot{\varepsilon} a \tilde{a} \lambda \omega \nu$ $\delta v_{o}$ ) periclitatus esse videri potest.

His locis igitur, etsi verum non vidit, aliquid tamen in emendando secutus est et recte errorem subesse perspexit. alibi autem sine causa verba Euclidis falsa ratus pravo iudicio mutavit, quae diligentius consideranti recte vel saltim cum excusatione iusta scripsisse Euclides videtur. cuius generis haec habeo exempla.

III 24 p. 127, 1 sq.

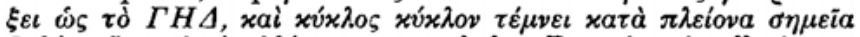

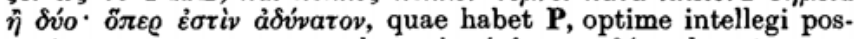

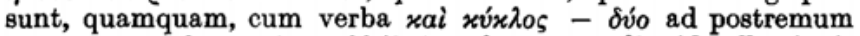
tantum membrum ( $\pi \alpha \rho \alpha \lambda \lambda_{a}^{\prime} \xi \varepsilon$ ) referantur, aliquid offensionis

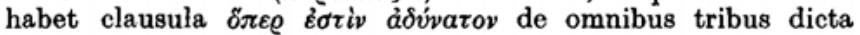
(cf. I p. 127 app. I ad 1). Theon tamen duobus primis membris ad

1) Euclidem non semper evitasse verbis a se definitis alio quoque sensu uti, demonstrat usus formulae $\delta \imath^{\prime}$ i $\sigma o v$ II p. 6 app. I ad 9;

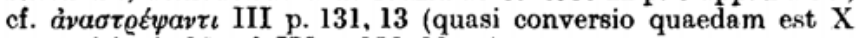
propositionis 16; cf. III p. 132, 11 sq.). 


\section{PROLEGOMENA CRITICA}

plenam demonstrationem necessariis deletis suo arbitrio ita locum refinxit $\dot{\alpha} \lambda \lambda \dot{\alpha} \pi \alpha \varrho \alpha \lambda \lambda \alpha^{\prime} \xi \varepsilon \iota^{\cdot} x \dot{v} x \lambda o \varsigma \delta \dot{\varepsilon} x \dot{x} x \lambda o v o \dot{v} \tau \dot{\varepsilon} \mu \nu \varepsilon \iota x \tau \lambda$.

Corollarium post V 7 ab Euclide apte et temperi collocatum iam e V 4 deducere posse sibi visus est Theon; quare ibi collocavit addita demonstratione non nimis adcurata, et tamen commodum, quod solum inde capi posset in demonstratione prop. VII, neglexit (II p. 12 app. I ad 21. 22).

Praeclarum exemplum mutationis temerariae habemus in VI 14;

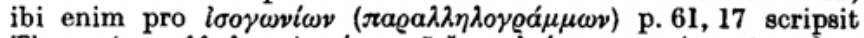

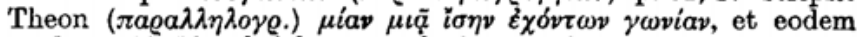
modo p. 61, 19 ad falsam analogiae speciem prop. 15 mutavit

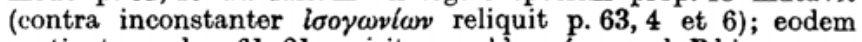

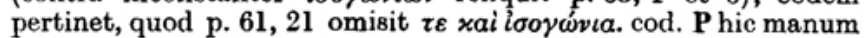
Euclidis retinere, demonstrat et scriptura Theonis VI 16 p. 66, 11 ; 67, 1 (ne dicam de p. 63, 4. 6) et Philoponus codicem $P$ sequens.

II p. 87, 14 quaedam omisit falsa figura deceptus; v. II p. 87 app. I ad 14. XI deff. 27 et 28 permutavit Theon polyedra secundum numerum planorum ordinans, cum Euclides aptius ea secundum genera planorum ordinavisset; cum $\mathbf{P}$ hic facit Psellus. $V$ deff. 6-7 maxime propter Campanum dubitari potest, an ordo codicis $\mathbf{P}$ genuinus non sit, quamquam per se aptior est ordine ex codd. Theonis recepto.

XI 1 Theoni displicuit locus p. 4, 19-21, ubi breviter et subobscure, sed recte ratio redditur, cur duarum rectarum diversarum pars communis esse non possit; quare ad axioma novum confugit (v. app. crit.; scriptura codicis $\mathbf{P}$ etiam in quosdam codd. Theoninos irrepsit).

XI 38 cum Euclides de solo cubo demonstravisset, quia hic casus oeconomiae Elementorum satisfaceret (v. IV p. 73 app. I ad 13), Theon recte observavit, eandem demonstrationem de quovis parallelepipedo valere (nec hoc Euclides non vidisse putandus est), non recte pro casu speciali generalem propositionem substituit pro xv́ßov scribens $\sigma \tau \varepsilon \varrho \varepsilon o \tilde{v} \pi \alpha \varrho a \lambda \lambda \eta \lambda \varepsilon \pi \iota \pi \varepsilon ́ \delta o v ~ p . ~ 73,13,15,17 ; 74,2$; $75,8,10$.

XII 7 coroll. non dubito, quin iam Theon scripturam imperfectam habuerit et ea ipsa de causa omiserit xai $\omega \varsigma-\tilde{\varepsilon} x a \sigma \tau o v$ p. 99, 12; eodem loco immerito omisit $\tau o \omega v \bar{\tau}$ lin. 10 et xai lin. 11 (nam scripturam falsam lin. 10 av̉zò $\tau \dot{c}$ vel $\tau \dot{o}$ av̉ंò nunc librariis, non Theoni imputo; cf. IV p. 99 app. I ad 9).

XII 17 cum intellegeret Theon, perpendicularem a $K$ ad $B \Phi$ in ipso $\Phi$ cadere, per reliquam demonstrationem a p. 132,1 pro $\Omega$ scripsit $\Phi$, sed non demonstravit, $K \Phi$ perpendicularem esse, nec vidit, Euclidem, cum nihil ad demonstrationem ipsam referret, utrum $K \Phi$ an $K \Omega$ sumeret, prudenter cavisse, ne demonstratio sine causa longior fieret; cf. IV p. 131 app. I ad 23.

Minora nec ad rem, sed ad verba sola fere pertinentia haec sunt:

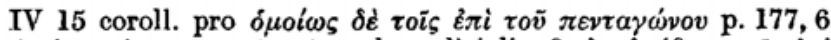

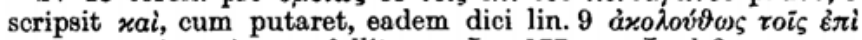

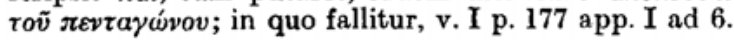




\section{PROLEGOMENA CRITICA}

$\mathrm{V}$ def. 10 ó $\mu$ oí $\omega$ s p. 2, 15 obscurum ei visum est (est autem satis clarum); quare reposuit obscurius źvi $\pi \lambda \varepsilon i o v \varsigma$ (sc. $\lambda \delta$ jovs) ex VIII 3 p. 153, 8.9 (cf. p. 13, 22) petitum.

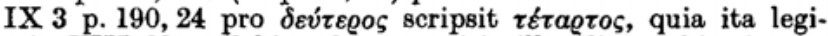
tur in VIII 23; sed hic, ubi propositio illa aliis verbis citatur,

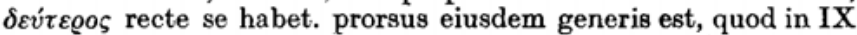

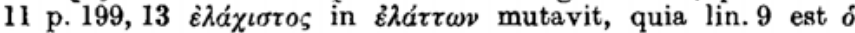

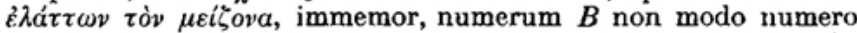
$E$ minorem esse, sed etiam ex quattuor $B, I^{\prime}, \Delta, E$ minimum. aliquatenus similis est correctio X 33 p. 56, 16, ubi $\sigma v ́ \mu \mu \varepsilon т \rho \nu$ in

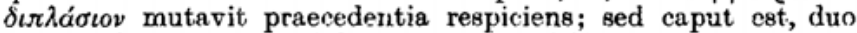
rectangula commensurabilia esse. etiam VIII 21 p. 183, 7 seripturam per se bonam $\delta E \tau \dot{\nu} \nu \Gamma$ in $\delta H \tau \dot{\partial} \nu B$ mutavit, quia hae litterae proxime et antecedunt ct sequuntur.

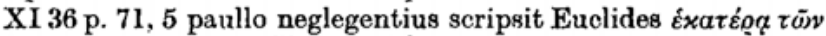

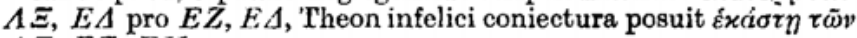
$\Lambda \Xi, E Z, E H$.

XII 3 p. 84, 16 iusto durius ex

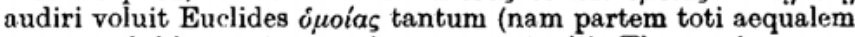
esse, nemini in mentem venire posse putavit); Theon minus confidenter de peritia lectorum iudicavit et $\delta \mu o i \alpha \varsigma$ diserte addidit.

IV 1 p. 152, 11 ov prorsus inutiliter inculcavit; nam optime sic

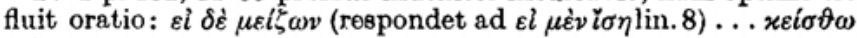
cet.

De scriptura $\dot{\varepsilon} \dot{\alpha} \nu$ I 13 p. 21, 2 Theoni tribuenda nunc dubito,

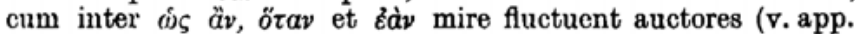
crit. et Studien p. 185), ita ut difficile sit diiudicatu, quid Euclides scripserit; I p. 22, 3 etiam $\mathbf{P} \dot{\varepsilon} \dot{\alpha} \nu$ habet.

His locis igitur errores deprehendere sibi visus est Theon; alibi orationis formam meliorem reddere mutando se posse puta. vit. et primum emendationes, si dis placet, ampliores has collegi:

VIII 3 p. 153, 14-19, ubi brevitati studuit.

IX 2 p. 189, 4. 5, ubi ad formam propositionis ipsius respexit.

IX 15 p. 206, 13 sq., ubi sic scripsit Euclides (cf. p. 206 app. I ad

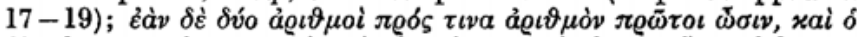

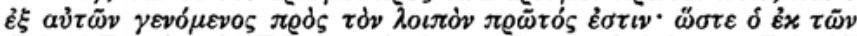

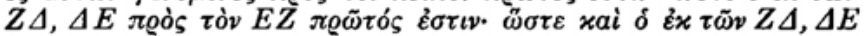

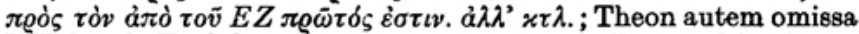
VII $24 \mathrm{ab}$ Euclide contra morem suum omnibus verbis citata:

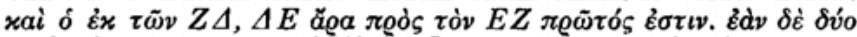

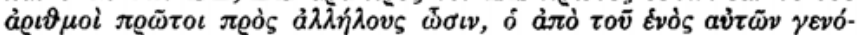

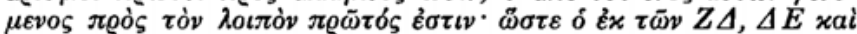

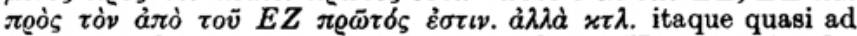
compensandam propositionem omissam aliam VII 25 citavit. alia exempla v. vol. III p. 127, 10sq., IV p. 80,5 sq., p. 90,20 sq. (v. append. I 4 p. 194), p. 95, 17 sq., p. 120, 13 sq. et minora cum additamentis (v. infra) coniuncta III p. 29,7 sq., p. 94, 14sq., IV p. 96,13 sq.; cf. etiam III p. 63,11 . 12. contra IV p. 141,24 sq. verba Euclidis in formam breviorem redegit; cf. IV p. 105, 15 sq. 


\section{PROLEGOMENA CRITICA}

Plerumque tamen mutationes illae ad pauca tantum verba minoresque sententiarum partes pertinent, quae aliqua de causa aliter conformare ei libuit, velut

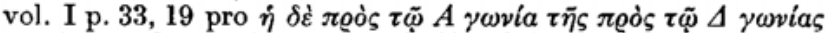

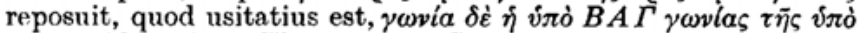
$E \Delta Z$; idem fecit II p. 49,10 ; IV p. 154,3 , cf. III p. 53, 21; contra II p. 51, 19. 20 illam formam restituit, sine dubio ad similitudinem p. 51,6 et 52,2 .

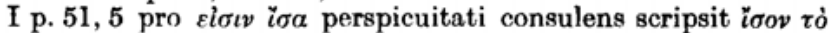

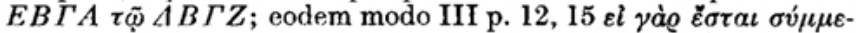

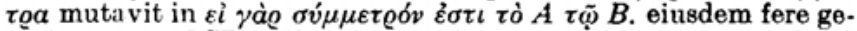
neris est, quod II p. 129,16 pro av่rov̀ s scripsit tov̀ $A, B$; cf. III p. 88,$19 ;$ p. 169,$14 ;$ p. 170,12 . contrarium factum videmus IV p. 115,18 , cf. I p. 132,5 ; II p. 46, 13. conferri potest etiam I p.177,

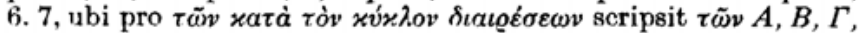
$A, E, Z$ $\sigma \eta \mu \varepsilon i(\omega v$. etiam I p. 167, 14 vellem recepissem scripturam

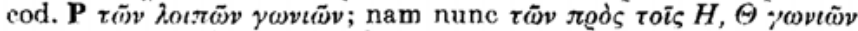
Theoni tribuendum esse video.

I p. 83, 1.2 pro $\check{\imath} \sigma \eta \delta \dot{\varepsilon} \dot{\eta} H Z \tau \tilde{\eta} \Gamma \Delta$ recipiendum erat e $\mathbf{P}$ :

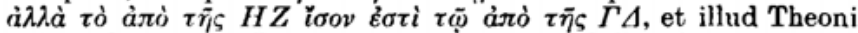
tribuendum. etiam I p. 88, 5. 6 scripturam cod. P et Campani nunc non dubitassem recipere; nam veri simile est, Theonem non modo

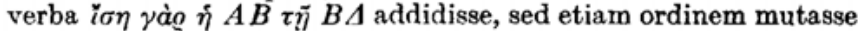
ad sequentia lin. 7 respicientem.

I p. 92, 12 quoque fieri potest, ut $\mathbf{P}$ verum praebeat $\tau \dot{\delta} v \pi \dot{\delta} \tau \tilde{\omega} \nu$ $B E, E \Delta$, et ut Theon ob sequentia lin. 13 rò $B \Delta$ scripserit, quamquam ob Campanum res incerta est.

I p. $147,17.18$ cum in aequatione pro $Z \Delta^{2}$ substituendum esset

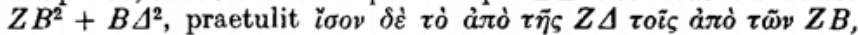

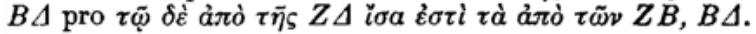

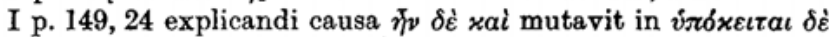
(contra II p. 226, 9.10 pro xai v́r $\delta x \varepsilon \iota \tau a \iota ~ \delta$ scripsit $\delta \delta \dot{\varepsilon}$, fortasse quia hoc non ab initio suppositum est, sed postea accessit). eadem

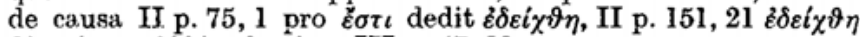

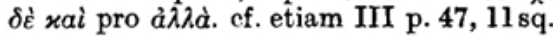

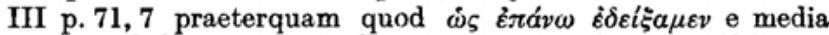
sententia ad finem removit, litteras permutavit et pro $\dot{\ell} \lambda a^{\prime} \sigma \sigma o v a$ posuit $\mu \varepsilon i \zeta o v a$, quia sub hac ipsa forma propositio demonstrata est in lemmate X 41.

III p. 174, 1.2 quia in proportione ordo est $A H, A H \times H B$, $H B$, ordinem verborum mutavit; aliquatenus similis est locus II p. $173,1-3$.

III p. 14l, 10 breviorem formam $\mu i \alpha$ ă $\alpha \varkappa \tau \lambda$. ad similitudinem propositionis ipsius (p. 140,10) pluribus verbis redegit. idem studium brevitatem Euclidis explicandi causa est, cur III p. 195, 8 pro $\hat{\eta}$ ov scripserit $\dot{\varepsilon} a v \tau \tilde{\eta} \hat{\eta} \tau \tilde{\varphi} \dot{\alpha} \pi \dot{d} \dot{a} \sigma v \mu \mu \varepsilon \dot{\varepsilon} \tau \varrho o v$. omnino saepius anxiae cuidam diligentiae inservit, quasi lectoribus aut fatuis aut

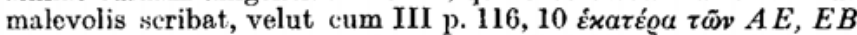

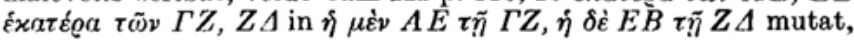




\section{PROLEGOMENA CRITICA}

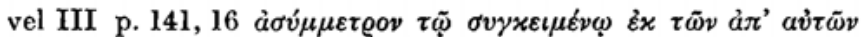

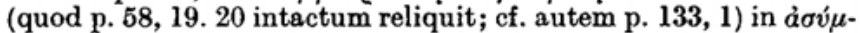

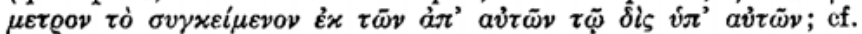
etiam III p. 162, 16. eodem pertinet, quod multis locis, ubi Eucli-

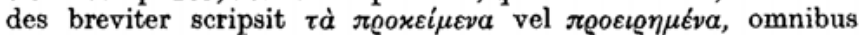
verbis expressit, quae hac formula commode significantur (v. III p. 66,$13 ; 131,7 ; 132,5 ; 133,5 ; 139,16 ; 140,16,19 ; 141,9,19$,

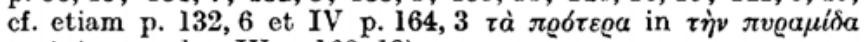
mutatum, sed v. IV p. 168,19$)$.

Etiam II p. 159, 25; III p. 1, 11; 126, 168q.; IV p. 95, 21. 22; 198, 16 mutavit, quia sic clarius ei proponi videbantur. et eodem referri potest II p. 29,$10 ; 48,5$, ubi $x a i \dot{\varepsilon}^{2} v \alpha \lambda \lambda a^{\prime} \xi$ in $\dot{\varepsilon} v \alpha \lambda \lambda \dot{a} \xi$ ăoa.

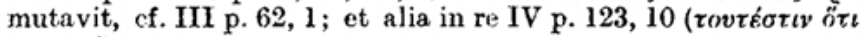
pro $x u i)$.

III p. 32, 2 ordinem verborum rectum, sed submolestum mutando commodiorem reddidit. idem fecit III p. $80,19.20 ; 147,13$, ubi $\tau \tilde{\eta} \Delta$ et $\tau \tilde{\eta} . A$ a $\oint \eta \tau \tilde{\eta}$ interiectis verbis $\sigma \dot{v} \mu \mu \varepsilon \tau \tau^{\prime} v \dot{\varepsilon} \sigma \tau \iota$ et $\sigma \dot{v} \mu$ $\mu \varepsilon \tau \varrho \circ \varsigma$ dirimi noluit. etiam III p. $28,16.17 ; 29,6$ verba $\sigma v ́ \mu$ $\mu \varepsilon \tau \varrho \sigma ́ \varsigma \dot{\varepsilon} \sigma \tau \iota$ ad finem reiicere placuit. et III p. 173,15 praetulit commune verbum $\dot{\varepsilon} \sigma \tau \iota$ ante periodum per $\mu \dot{\varepsilon} \nu$ et $\delta \dot{\varepsilon}$ divisam poni quam in primo membro; itaque I p. 129, 15, ubi eadem prorsus ratio est in $\tilde{\varepsilon} \sigma \tau \omega \sigma \alpha v$, scriptura codicis $\mathbf{P}$ recipienda erat, recepta Theoni tribuenda. sed hic necessario multa dubia sunt. cf. etiam II p. 105,8 sq.

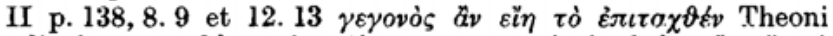
displicuit ut problemati aptius; quare scripsit $\delta \tilde{\eta} \lambda o v$ àv $\varepsilon \grave{\eta} \eta \tau \dot{o}$ $\zeta \eta \tau o v \mu \varepsilon v o v$; idem fecit II p. 139, 6. 7. Euclidis in verbis eligendis iudicium item improbavit III p. 47, 17. 18; 110, 8; IV p. 96, 7. 8; cf. etiam III p. 25, 23. etiam in vocabulis mathematicis haud ita raro usum Euclidis sine causa vel etiam cum damno reliquit. velut

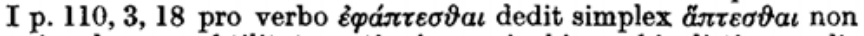
animadversa subtilitate antiquiorum in his verbis distinguendis (v. I p. 122 app. crit. ad 1); cf. I p. 166,3 (áq

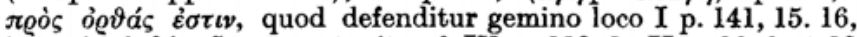

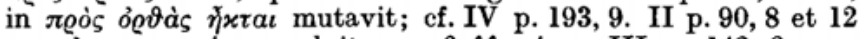

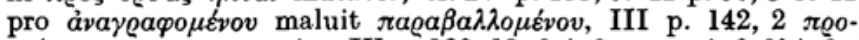

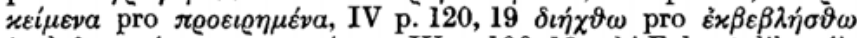

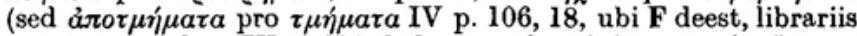

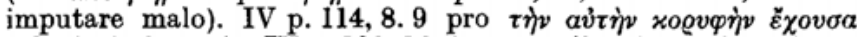

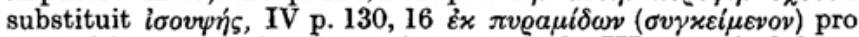

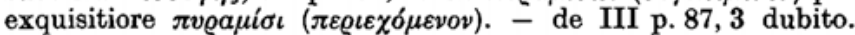

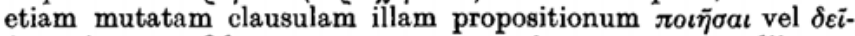
gal minus confidenter commemoro, quia saepe compendiis am. biguis scribebatur, et interdum unus et alter Theoninorum cum $P$ congruit, velut I p. 129, 6; ibi enim non dubito, quin ex PF recipiendum sit $\delta \varepsilon \bar{\imath} \xi \alpha$, quia $\pi o ́ \rho \iota \sigma \mu \alpha$ est, v. Studien p. 61 ; item in I p. 95, 16 e $P$ recipiendum $\delta \varepsilon i \xi a u$, sicut factum est II p. 110, 3 (cf. p. 108,8 ). omnino in omnibus propositionibus, quas l. 1. p. 61 porismata esse significavi, nunc ex omnibus vel saltim (ut in X 3 et 4) 


\section{PROLEGOMENA CRITICA}

e melioribus codd. $\delta \varepsilon i \tilde{\xi} a \iota$ restitutum est exceptis VI $11-13$, quam-

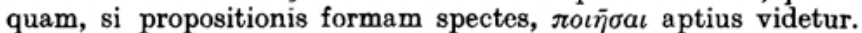
unde opinio mea de porismatis haud mediocriter confirmatur. etiam in X $27-35,48-53,86-90$, quas l. l. p. 62 vix recte e numero porismatum seclusi, nunc fere $\delta \varepsilon \bar{\imath} \xi a \iota$ legitur, sed plerumque exigua auctoritate, cum clausula illa plerumque in codd. Theoninis omissa sit, in $\mathbf{P}$ compendio scripta; III p. 146, 4 in omnibus codd.,

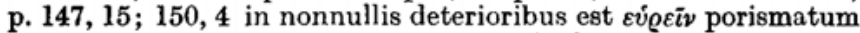
proprium (Studien p. 62). magis etiam de I p. 18, 16 dubito, ubi $\mathbf{P}$ solus $\delta \varepsilon \bar{i} \xi a \iota$ habet ( $\gamma \varrho . \pi \circ \imath \tilde{\eta} \sigma a \iota \mathrm{mg} . \mathrm{m} . \mathrm{l}$ ), ut in simili propositione I p. 135,14 . IV p. $18,6,24 ; 37,7 ; 47,3 \delta \varepsilon \bar{\xi} \xi \alpha \iota$ vix defendi potest, quamquam dubitandi locum relinquit et consimilis ratio harum propositionum et in duabus ultimis consensus unius vel etiam plurium Theoninorum. IV p. 133, 7 Theon forma conclusionis permotus $\delta \varepsilon i \xi \alpha \iota$ pro $\pi \circ \imath \tilde{\eta} \sigma \alpha \iota$ scripsisse videtur.

Hoc in genere pono etiam, quod interdum litteras figurarum permutavit, velut III p. 85,3 sq. ad similitudinem prop. 49 et fortasse etiam IV inde a p. 96,9 . cf. etiam vol. III p. 90,9 ( $A B \Gamma \Delta$ pro breviore $A \Gamma)$, IV p. 78, $5(A B \Gamma \Delta E, Z H \Theta K A$ pro $A B \Gamma$, $Z H \Theta$ ), IV p. 171,22. de I p. 132, 6 dubito, quia ibi $\mathbf{F}$ plerumque cum $\mathbf{P}$ consentit; quare mutatio Theone posterior videtur.

Interdum ad minutias sermonis putide corrigendas more magistellorum ineptorum descendit, velut cum in hac formula $\delta$ เ $\varrho \eta^{\prime}$ -

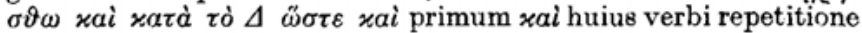
offensus constanter omittit (III p. 69, 1. 2; 71, 4; 73, 14;74, 16

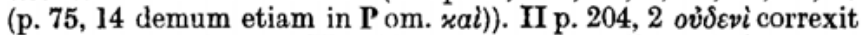

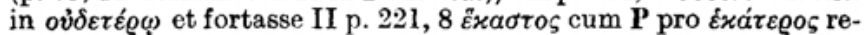
tinendum, cum Euclides in talibus rebus parum religiosus sit. IV

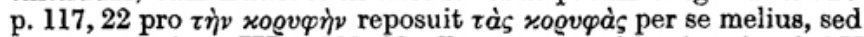
non necessarium. III p. 199, 23 offensus est verbis $\tau \dot{\text { ò }} \mu \varepsilon \bar{\zeta} \zeta \mathrm{ov} \dot{\eta} \Delta H$ et reposuit $\dot{\eta} \mu \varepsilon i \zeta \omega \nu \hat{\eta} \Delta H$ (si testimonio codicis $\mathbf{B}$ credimus); sed auditur övo $\mu \alpha$. cum hoc loco conferri potest II p. 96, 9, ubi $\tau \mu \tilde{\eta} \mu \alpha$ $\dot{\eta} A E$ (pro $\tau \dot{o} A E$ Theoninorum) fortasse cum $\mathbf{P}$ retinendum. II p. 164, 19 submolestum $\dot{\varepsilon} x a \tau \varepsilon \varrho o v$ delevit tamquam minus necessarium. saepius pro participio temporis praesentis usitatius perfecti scripsit, velut III p. 235,8 ; IV p. 156,$18 ; 179,18$ et 20 (ubi scripturam cod. P receptam esse oportuit); cf. III p. 124, 2. saepe etiam futurum praesenti practulit, velut in $\tau \varepsilon \dot{\varepsilon} \mu \nu \varepsilon \iota \mathrm{I}$ p. 97, 3.

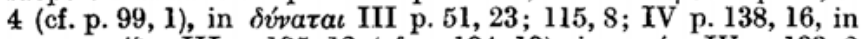

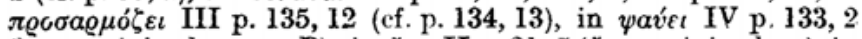
(hoc recipiendum ex P), in $\check{\varepsilon} \chi \varepsilon \iota$ II p. 21, 5 ( $\check{\varepsilon} \chi \varepsilon \iota$ recipiendum), in $\mu \varepsilon \tau \varrho \varepsilon \bar{\imath}, \mu \varepsilon \tau \varrho \circ \bar{v} \sigma \iota$ II p. 107,$25 ; 144,4 ; 227,7$, ubi nunc praesens cum $\mathbf{P}$ probo (et omnino librarii quoque saepe futurum inculcaverunt, velut II p. 172, $24 \mathrm{BV}$; II p. 174, $4 \mathrm{Vb}$; III p. 7, $20 \mathrm{P}$; II p. 144, 12; 145, 1, 4, 9, 13, 14 P; III p. 136, 15 P; de II p. 160,11 et III p. 202, 15 dubito). eodem modo in $\varepsilon \sigma \tau \iota-\tilde{\varepsilon} \sigma \tau a \iota$ variatur; I p. 54,

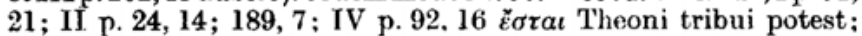
III p. 2, 9 غ̇ $\sigma \tau \iota$ nunc mibi verum videtur collato III p. 4,9; etiam

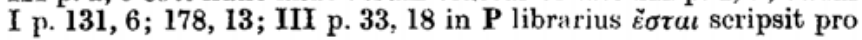

\section{XLIV}




\section{PROLEGOMENA CRITICA}

$\dot{\varepsilon} \sigma \tau \iota$; de II p. 194, 6; III p. 190, 13; IV p. 111,5 rem in medio relinquo. Theoni autem sine dubio tribuendum $\pi \varepsilon \varrho \iota \varepsilon x \eta$ bis pro

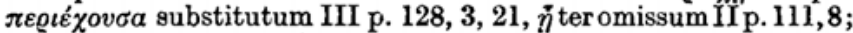
115,$21 ; 116,28$ (sed pepercit p. 112,10), ä $\alpha$ ter retractum III p. 88, 3; 135, 19; IV p. 47, 1 et fortasse etiam I p. 101, 19; IV p. 133, 17. 18 (cf. transpositio eiusdem particulae III p. 7, 18. 19 et loci memorabiliores infra allati), bis $\varepsilon \check{\tau} \iota \tau \varepsilon$ pro $x a \grave{\iota} \varepsilon \check{\tau} \iota$ III p. 133,$1 ; 143,16$, numerus pluralis saepius pro singulari post subiecta neutrius generis substitutus I p. 54, 4; III p. 10, $5^{1}$ ); III p. 2,2 ; IV p. 6,$6 ; 128,1 ; 167,8$; III p. 235,3 (cf. IV p. 137.

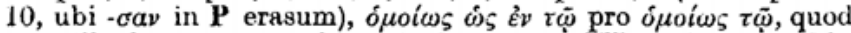
nota illa Graecorum neglegentia dictum est, III p. 50, $7, \dot{\omega} \zeta$ additum III p. 206, 13. minus certa et levissima fere sunt, quae his locis mutata sunt: I p. 3,$18 ; 148,9$; II p. 11,$11 ; 20,20,21$; III p. 31,$14 ; 46,13 ; 71,6 ; 117,15 ; 141,15 ; 160,10 ; 190,1 ; 205,20$; IV p. 101, 16.17; 113,20;121, 2, quorum maximam partem Theoni tribuerim. contra $o$ ter male additum II p. 103,$11 ; 104$, 21, 23 librariis, non Theoni debetur. quoniam secundo loco $\mathbf{B}$ cum $P$ in eo omittendo consentit. III p. 116,$11 ; 232,5$ xai pro $\delta \dot{\varepsilon}$ Theoni tribueris, sicut fortasse etiam I p. 139, 12; sed III p. 67,18;101,2 $\delta \dot{\varepsilon}$ in Theoninis est, $x a i$ in P. IV p. 29,$19 ; 31,4 \hat{\eta}$ a Theone bis additum puto ad evitandam constructionem durissimam $\dot{\varepsilon} \lambda a \sigma \sigma o ́-$ $\nu \omega \nu \tau \varepsilon \sigma \sigma a ́ g \omega \nu$; itaque fortasse etiam IV p. 185, $17 \pi \lambda \varepsilon t o ́ v \omega \nu \hat{\varepsilon} \xi$ $\gamma \omega \nu l \tilde{\omega} \nu$ ferri potest. II p. 153, 9 pro $\tilde{\varepsilon} \omega \varsigma$ cum coniunctivo in Theoninis est $\tilde{\varepsilon} \omega \varsigma$ ov, sed cum utrumque in Elementis reperiatur ( $\tilde{\varepsilon} \omega \varsigma$ III p. 211, 14, $\tilde{\varepsilon} \omega_{\zeta}$ ov III p. 4,9;IV p. 93,11), resincerta est; comparandum tamen, quod IV p. 5, 19 pro $\varepsilon i . . \not h$ in Theoninis est $\varepsilon i . .$. $\varepsilon i \eta$. II p. 206, 15; III p. 29, 7 in locis alio quoque modo a Theone mutatis o $\mathbf{P}$ receptum est $\tilde{\omega} \sigma \tau \varepsilon$ pro $\tilde{\alpha} \varrho \alpha$, et idem IV p. 38,4 factum esse potuit; sed obstant II p. 146, 6; III p. 198, 22, ubi đ̆o $\alpha \mathbf{P}$, $\tilde{\omega} \sigma \tau \varepsilon$ Theonini (loco posteriore ắ $\alpha$ recepi propter V), id quod ostendit, in hac re arbitrium librariorum, non voluntatem editoris regnare. eadem inconstantia est in ai $\delta \dot{\varepsilon}$ et xai $\varepsilon ̌ t \iota$ ai post ai $\mu \dot{\varepsilon} \nu$ permutandis; nam IV p. 30,$12 ; 45,17 \delta \dot{\varepsilon} \mathbf{P}$, xai ž $\tau \iota$ Theonini, IV p. 33, 3 жai ह̌ $\iota \mathbf{P}, \delta \varepsilon$ ' Theonini; fortasse ubique rarius et insolentius xai $\check{\varepsilon} \tau \iota$ (post $\mu \dot{\varepsilon} \nu$ ) praeferendum, quod IV p. 34, 17 in omnibus codd. est. III p. 114, 9 ह $\pi \varepsilon i$ a Theone additum esse potest; sed cum a librario cod. $P$ bis in $\varepsilon \pi \varepsilon i$ omittendo erratum est (III p. 94, 13; IV p. 117, 2), hic quoque errorem supponere licet. II p. 207, 6 vंxó pro rariore, sed in numeris recto $\dot{\varepsilon} x$ a librario, non a Theone substitutum est; nam II p. 207, 8 b cum $P$ facit, et etiam IV p. 70, 7 a

1) Neque tamen praetermittendum est, saepius etiam in Theo-

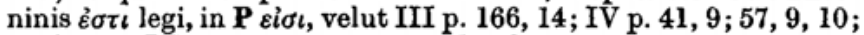

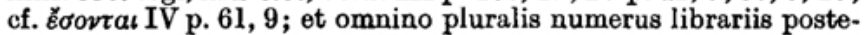
rioribus proclivior est; cf. IV p. 41, 13, ubi $\dot{\varepsilon} \sigma \tau \iota$ in $\mathbf{P}$ in $\varepsilon l \sigma \iota$ cor-

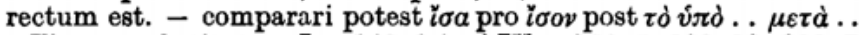
a Theone substitutum I p. 148, 9 (sed IV p. 188, 5; 199, 12; 200, 7 pluralis in plerisque est). 


\section{PROLEGOMENA CRITICA}

librariis nonnullis falso $\dot{v} \pi \dot{o}$ pro $\dot{\varepsilon} x$ scriptum est; cf. II p. 206, 20 Vq. contra II p. 206, 18 à $\dot{\partial}$ pro $\dot{\varepsilon} x$ non sine causa Theoni tri-

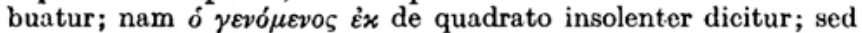
de toto loco aliter iudicandum est, v. supra p. XLI. postremo loco rem pertractabo, quae paullo latius patet. ubi ad demonstrationem rei alicuius propositae adiungendam transitur, saepissime $\dot{\varepsilon} \pi \varepsilon i$

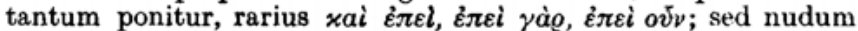
illud $\dot{\varepsilon} \pi \varepsilon \dot{l}$ librariis displicuit, qui vel $x a l$ vel $o v v$ vel $\gamma a \dot{\alpha} \varrho$ plurimis locis addiderunt (v. I p. 65, 20;117, 14; II p. 11, 1;94, 9; 129, 21; 130,$17 ; 132,14 ; 136,18 ; 145,3 ; 156$, 10; III p. 58,$1 ; 94,11 ; 97$, 6 ; 105,7 ; IV p. 63,$19 ; 67,10 ; 97,17 ; 143,23 ; 150,8 ; 152,11$; 200,18 ). $\mathbf{P}$ his locis plerumque cum optimo quoque Theoninorum interpolationis manifestae expers est; est tamen, ubi librarius peccaverit (II p. 129, 21 $\gamma$ da $\varrho$ add., III p. 97, 6 xai); itaque II p. 158, 18 xai cum Theoninis delendum, fortasse etiam I p. 134, 3 cum $\mathbf{B} \mathbf{p}$, sed hic $\mathbf{F}$ cum $\mathbf{P}$ facit. quoniam igitur in his formulis a librariis toties variatur (cf. praeterea I p. 141, 17; II p. 36, 13; 188, 11; III p. 160, 11), difficile est diiudicatu, num غ̇лci oṽv ter pro xai ėлci substitutum (I p. 166, 2; II p. 48, 6; III p. 39, 24) re vera Theoni ipsi tribui possit, quod feci III p. 39, idque eo magis,

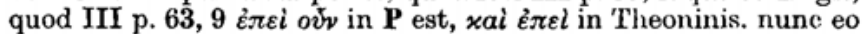
inclina verim, ut omnibus locis, ubi codices fluctuent, غ̇лદi restituendum esse putem. etiam de addito ovv I p. 122, 7; II p. 37, 11; III p. 48, 15 vel xai II p. 8, 7; IV p. 141, 6 vel $\gamma$ à $\varrho$ III p. 190, 2 caute iudicandum est; nam haec veri similius librariis quam ipsi Theoni tribuuntur.

In hoc toto genere mutationum ad orationis formam spectantium hoc praecipue Theon studuit, ut omnia, quae proprii aliquid haberent et a solita forma abhorrerent, mutando tolleret et ad unam eandemque quasi normam exigeret. huc iam ex locis proxime allatis unus et alter spectat, sed magis perspicuum hoc eius studium est in exemplis, quae sequuntur:

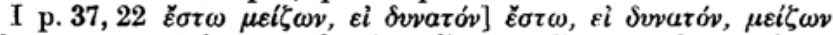
Theon, quae est forma vulgaris. solitum ordinem verborum item restituit I p. 108, 4; III p. 104, $\left.24 ; 110,12 ; 112,22.23 ; 169,2^{1}\right)$; IV p. 104, 9; 105, 17 (cí. app. I ad 17); de II p. 87, 5. 6; 191, 6; III p. 29,3 ; IV p. 38,$5 ; 86,3.4 ; 104,22$ Theoni tribuendis dubito. I p. 163, 14 vero nun dubito, quin Theon ordine insolito, sed probo (cf. verbi causa I p. 168, 22; 173, 21; 174, 23; 177, 1; IV p. 45,1 ) offensus ă $\alpha$ transposuerit in eum locum, quo est I p. 153,$22 ; 155,12 ; 159,4 ; 162,6 ; 164,18$ al.; miror, cur non idem fecerit I p. 152, 18; 157, 5; 160, 21. ne III p. 207, 10 quidem du-

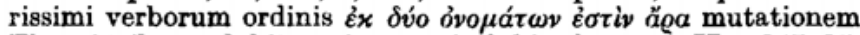
Theoni tribuere dubitaverim; magis dubius locus est II p. 147, 15,

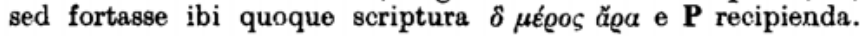

1) Cf. III p. 166, 13; 171, 11. alia orationis forma est p. 160, 5; $163,14$. 


\section{PROLEGOMENA CRITICA}

Eadem ratione in verbis eligendis nova omnia evitat et tollit,

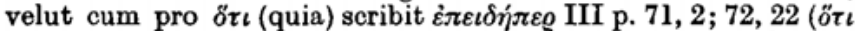
in hac significatione nusquam alibi, quod meminerim, in Elementis occurrit, sed Euclides omnino ad partem non mathematicam sermonis sui parum attendit nec legibus putidis se adstrinxit, quod horum locorum causa, quos hic tractamus, semel dictum sit), $\delta เ \pi \lambda a \sigma i \omega v$ pro $\delta \iota \pi \lambda \tilde{\eta}$ IIl p. 58, 7, quod multo rarius est, xoเvò $v$ $\pi \varrho \circ \sigma \times \varepsilon i \sigma \vartheta \omega$ pro $x o \iota v \partial \dot{\nu} \delta \dot{\varepsilon}$ prorsus insolito II p. $89,15, \dot{\alpha} \lambda \lambda \dot{\alpha} \delta \dot{\eta}$, vulgarem ad propositionem conversam transitum (v. verbi causa II p. 173, 14), pro $\pi a ́ \lambda t v \delta \grave{\eta}$ II p. 172,6; cf. $\delta \dot{\eta}$ additum in simili

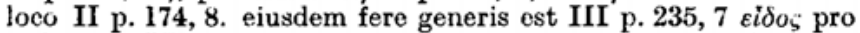

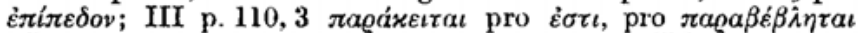
III p. 106, 22 (cf. 25); 173, 6 (cf. 10); $\gamma \dot{\alpha} \varrho$ pro $\delta \dot{\eta}$ III p. 113, 9 ad

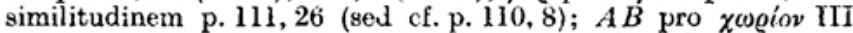
p. 172,9 (ut p. 167,$21 ; 169,22$ al.); ô $\pi \varepsilon \varrho$ in clausula theorematum notissima pro $\hat{d}$, quod hic tantum reperitur, sed non sine cau.

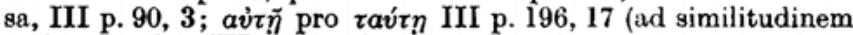

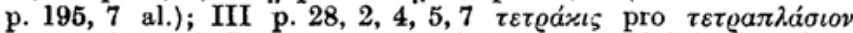
propter lin. 2,$3 ;$ I p. 52,3 pro insolito $x a \tau \dot{\alpha}$, quod defenditur loco simili I p. 154, 7, vulgare $\dot{\varepsilon} \pi$. magis dubii hi loci sunt: II p. 185, 4; III p. 11, 1; IV p. 38, 3, ubi in P error esse potest; cf. etiam II p. 59,$9 ; 204,12$. à $\pi \dot{o}$ pro غ̇ंi falso (post perfectum) substitutum IV p. 108, 18; 110, 20 non Theoni imputo, sed librariis etiam alibi illud praeferentibus (IV p. 111, 11 q; p. 114, 8; 115, 2 V). fieri potest, ut I p. 53, 7; 54, 8; II p. 43, 4; III p. 7, 2 (hic quidem in $\mathbf{P}$ errore scribendi $\delta \dot{\varepsilon}$ est) e $\mathbf{P}$ recipienda sit $\delta \dot{\eta}$ particula; nam cum rarior sit exceptis certis quibusdam formulis $(\lambda \dot{\varepsilon} \gamma \omega \delta \dot{\eta}$, $\delta \mu o i \omega \varsigma \delta \dot{\eta}$ al.) Theon fortasse vulgatius ă $\varrho a$ restituit; cf. III p. 198, $5(\delta \grave{\eta}$ P, ovv Theonini). si codicibus in talibus minutiis fides est, II p. 25, 8 pro xai $\varepsilon i$ scripsit $x a ̂ y$, quia ita est lin. 7 (sed v. p. 21, 24 al.).

Aliquanto maiora et fere ad constructionem sententiarum formasque verborum pertinentia haec sunt:

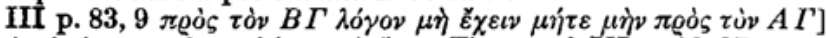

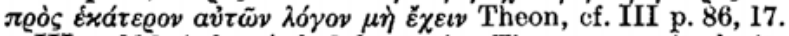

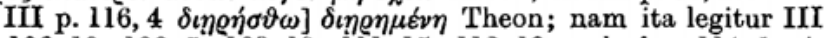
p. 106,$18 ; 108,5 ; 109,19 ; 111,15 ; 112,19$; sed ef. p. 114, 1. si-

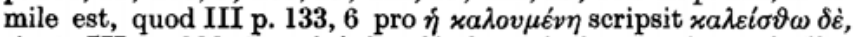

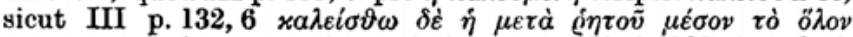

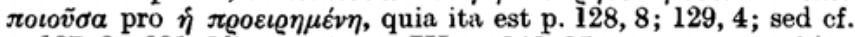
p. 127,$\left.3 ; 131,8^{1}\right)$. praeterea III p. 140,15 pro $\pi \varrho \circ \sigma \alpha \varrho \mu о \zeta \varepsilon ́ \tau \omega$ scripsit $\pi \varrho \circ \sigma a \varrho \mu \delta \zeta o v \sigma a$, sicut est p. 137, 2; 139, 10; sed cf. p. 135, 19 (alia rursus forma est p. 134, 15; 141, 18; unde apparet, quam non sibi constet Euclides in minutiis). III p. 185, 12 durum et insolitum d av́ $\mu \mu \varepsilon \tau \varrho o v$, quod significat $A H^{2}+H B^{2}$ unam magnitudinem esse, in $\dot{\alpha} \sigma v ́ \mu \mu \varepsilon \tau \varrho \alpha$ mutavit. etiam I p. 67,$12 ; 68,21 \tau \tilde{\varphi}$

1) $x a \lambda \varepsilon i$ ra pro $x a \lambda \varepsilon l \sigma \vartheta \omega$, quod in hac formula alibi semper legitur, e P III p. 60, 11; 65, 13 recipere non audeo, sed p. 128,8 ferri possit. 


\section{PROLEGOMENA CRITICA}

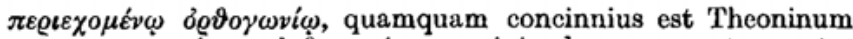

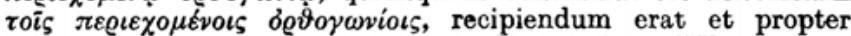
constantiam discrepantiae et propter I p. $69,3,20$. III p. 3, 7, 10 pro $\hat{\eta} \tau \dot{\partial} \tilde{\eta} \mu \iota \sigma v$ scripsit $\tau o \tilde{v} \dot{\eta} \mu i \sigma \varepsilon \omega \varsigma$ ad proxime praecedens $\varepsilon \dot{\imath} \lambda \alpha \sigma$ бov $\tau o \tilde{v}$ i $\mu i \sigma \varepsilon \omega \varsigma$ p. 3,6.7 accommodatum; contra IV p. 132, 6 fortasse e $\mathbf{P}$ recipiendum est $\delta \iota \pi \lambda a \sigma i o v$ pro $\ddot{\eta} \delta i \pi \lambda a \dot{\sigma} \iota v v$; nam eo

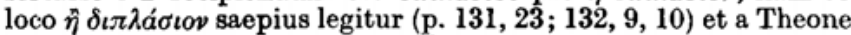

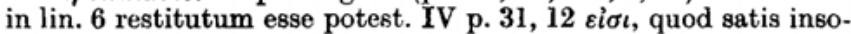
litum est (cf. verbi causa IV p. 33, 12 et I p. 31,5), in $\check{\varepsilon} \sigma \tau \omega \sigma \alpha \nu$

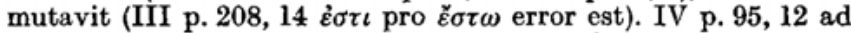
verba propositionis ipsius p. 95, 11 accommodavit. III p. 225, 14. $15 \tilde{\omega} \sigma \tau \varepsilon \ldots \dot{\varepsilon}^{\prime} \sigma \tau \iota$ pro $\tilde{\omega} \sigma \tau \varepsilon \ldots$. . Eival Theoni tribuendum videtur, quia indicativus longe frequentior est; tum etiam II p. 15, $17 \mathrm{cum}$

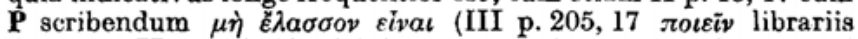
debetur). II p. 209, 10 vicinitas praesentis $\mu \varepsilon \tau \varrho \varepsilon \tau$ toties repetiti

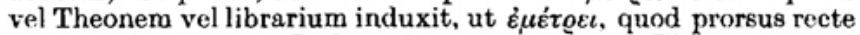
dicitur (cf. p. 18 app. I ad 17), in $\mu \varepsilon \tau \varrho \varepsilon i$ mutaret; II p. 18, 17 qui-

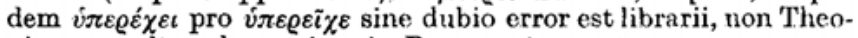
nis; nam altero loco etiam in B p servatum est.

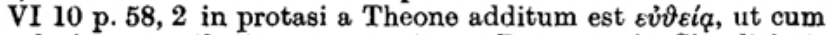
conclusione p. 59, 4 congruat (cum $\mathbf{P}$ consentit Simplicius). eodem modo XIII 12 p. 159, 22 consensum protaseos et conclusionis restituit, fortasse etiam X 81 p. 139, 2; XI 11 p. 16, 15 et praeterea II 7 p. 78, 11; II 8 p. 81, 1, quos locos confirmat simili-

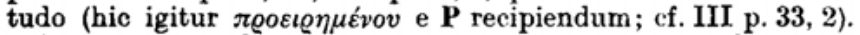

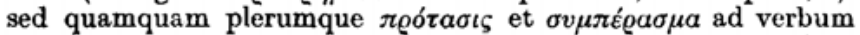
congruunt, tamen est, ubi plus minusve inter se discrepent (v. I 43, 47; III 11, 12, 13, 15, 20, 31; VI 3 ${ }^{1}$, 27; XI 14; XII 3).

Praeterea et addendo et omittendo normam regulamque sermo-

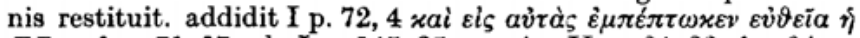
$\Gamma B$; ef. p. 71, 17 al. I p. 145, 25 xoเvòv. II p. 24,22 $\lambda \eta \varphi \vartheta \varepsilon ́ v \tau \alpha$

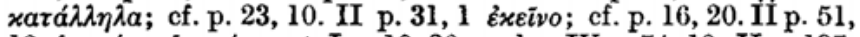

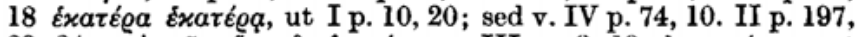

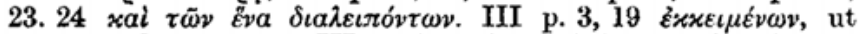

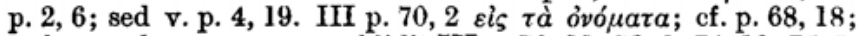
eadem verba supervacua addidit III p. 70, 19; 73, 8; 74, 10; 75, 7 .

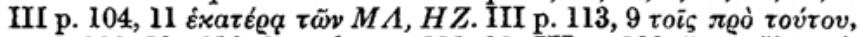

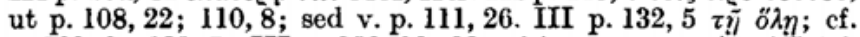

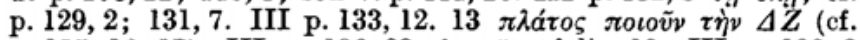

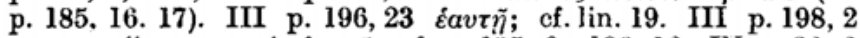

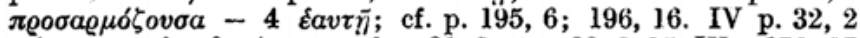
$\pi \alpha ́ v \tau \eta \mu \varepsilon \tau \alpha \lambda \alpha \mu \beta a v o ́ \mu \varepsilon v a l$; cf. p. 31,8 et p. 28, 8, 15. IV p. 176, 17

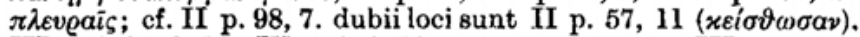
III p. 213, 13. 14; IV p. 140, 18 nec prorsus certus III p. 188, 5 ḋлото $\mu \eta-7 A B$ (notandum tamen, quod etiam lin. 8 in Theo-

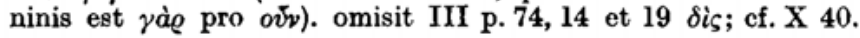

1) Cum hoc loco (II p. 44, 20) conferri potest I p. 23, 12 ubi cum codd. $\pi \circ \iota \eta^{\sigma} \sigma o v \sigma \iota v$ retineri potest ( $\pi \circ \iota v \bar{\sigma} \sigma v$ Proclus et I p. 24, 9).

\section{XLVIII}




\section{PROLEGOMENA CRITICA}

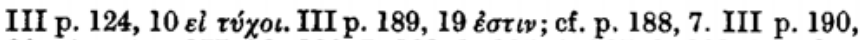
$20 \gamma d \varrho$, ut p. 177,$16 ; 180,7 ; 183,1 ; 185,4 ; 187,9 ; 189,8$; sed v.

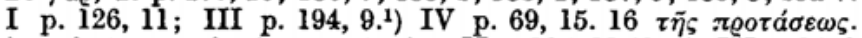

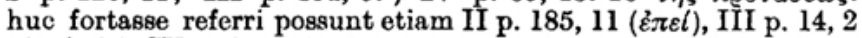
$(\dot{\alpha} \varrho \iota \vartheta \mu \partial \nu)$, IV p. $155,10(\tilde{a} \varrho \alpha)$.

Ex locis hic allatis comparatisque apparet, Theonem saepe illum quidem, sed non semper solitam orationis formam restituisse. si quis putet, me nimis inique de Theone iudicare hane inconstantiam ei imputantem, conferat, quae iam allaturus sum exempla, quae tam multa tamque inter se similia sunt, ut de casu aliquo cogitari non possit:

I p. 59, 3 pro $\dot{\varepsilon} \nu \varepsilon \dot{\tau} \varepsilon \sigma \varepsilon \nu$ scripsit $\varepsilon \mu \pi \varepsilon \dot{\varepsilon} \tau \omega x \varepsilon v$, quia hane formam hucusque solam habuit Euclides (p. 43,2;44,20;46,4); sed

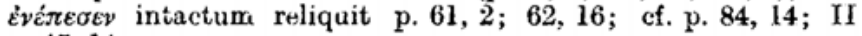
p. 45,14 .

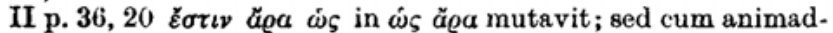
verteret, illam formam rursus p. $37,13.14 ; 41,24 ; 43,7$ occurrere,

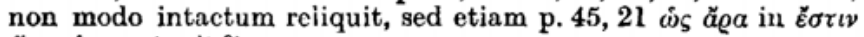
ă $\alpha \dot{\omega} \varsigma$ mutavit. $\left.{ }^{2}\right)$

I p. 157, 4 omisit é $\gamma \gamma \varepsilon \gamma \varrho a ́ q \vartheta \omega$ ஸ́ $\delta$ o $Z H E$, p. 159, 3 addidit

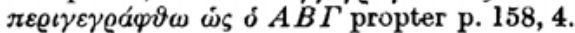

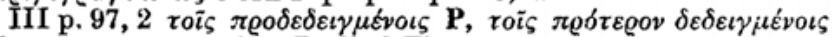
Theon, at p. 100, 1 hoc $\mathbf{P}$, illud Theon.

III p. 115, 18 xai av่t ̀े omisit, quamquam legitur p. 113, 18; p. 117,9 reliquit, p. 119,$6 ; 192,7$ addidit (p. 119,26 et $\mathbf{P}$ et Theon).

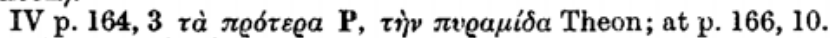
$11 \tau \dot{\eta} \nu \pi v \varrho a \mu l \delta a \mathbf{P}, \tau \dot{a} \pi \varrho \delta \tau \varepsilon \varrho o \nu$ Theon. fortasse huc referri possunt loci, quos p. XLV de ai $\delta \dot{~}$ et xai ह̌ $\tau \iota$ ai collegi; cf. enim IV p. 31,$14 ; 34,5$.

Similis inconstantia est, quod I p. 158,1 pro $A, B, \Gamma$ substituit $Z A, Z B, Z \Gamma$, p. 156,$15 ; 157,1 ; 163,4,11$ autem reliquit, et quod

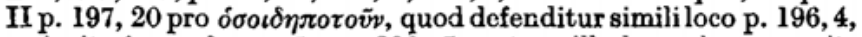
scripsit ó $\pi \circ \sigma o \iota \delta \eta \pi 0 \tau o \tilde{v}$, p. 200, 5 autem illud pro hoc recepit;

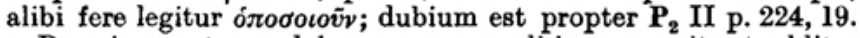

Praecipuum tamen laborem recensendi in eo posuit, ut additamentis lacunas, quas deprehendere sibi visus est, expleret ratiocinationemque Euclidis, ubi breviter intermediisque omissis exposita erat, suppleret planioremque redderet.

Primum igitur propositiones totas, quarum locum et usum esse putavit, interpolare non dubitavit, quale est additamentum eius in VI 33 (cf. II p. 102 app. I ad 4 et append. p. 233), de quo ipse gloriatur comm. in Ptolem. I p. 201. sed etiam in libro VII propositionem, quae vulgo est vicesima secunda (II append. p. 236),

1) Itaque etiam I p. 129, 14; II p. 197, $20 \gamma \dot{\alpha} \rho$ cum $\mathbf{P}$ retineri oportuit; cf. II p. 67,$24 ; 148,5$.

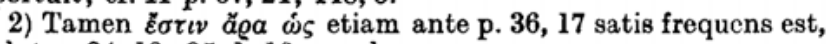
velut p. 34,$12 ; 35,1,16$, no plura. 


\section{PROLEGOMENA CRITICA}

addidit, fortasse etiam II p. 235 (vulgo VII 20), quamquam hoc propter B incertum est. ${ }^{1}$ ) in VI 27 casum alterum addidit (cf. II p. 231), post X 12 lemma (v. III append. 5 p. 216), II 4 et III 16 corollaria; utrum etiam V 19 et VI 20 corollaria, quae $\mathbf{P}$ in mg. a manu prima habet, a Theone addita sint necne, dubitari potest, maxime propter XII 8, ubi corollarium, quod et ipsum in $\mathbf{P}$ in $\mathrm{mg}$. est a manu 1, ab Euclide vix omissum erat; v.p. LXIV. eadem de causa de origine definitionis 5 libri VI dubitari potest; v. II p. 39 app. I ad 12. 13. certius videtur, demonstrationes alteras, $a \lambda \lambda \omega \omega \varsigma$ quae vocantur, hic illic a Theone interpolatas esse (velut in II 4 p. 181, VII 31 p. 237, et fortasse etiam in X 1 p. 211,6 p. 212, 9 p. 213, quae tres demonstrationes in $P$ in mg. a manu l postea additae sunt), quamquam pleraeque antiquiores sunt.

Cum eo genere mutationum, quod supra p. XLII sq. commemo-

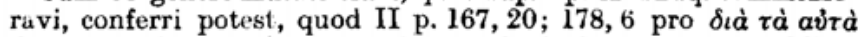
demonstrationem plene repetitam substituit. ceterorum additamentorum ampliorum haec genera distinguo:

Explicationes bonas illas quidem, sed parum necessarias addidit

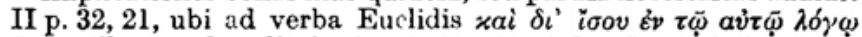

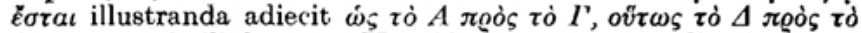
$Z$; prorsus similis locus est II p. 81, 18, suppares explicationes per

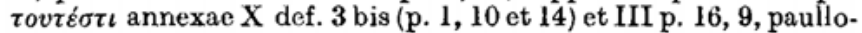
que aliter II p. 96, 5; III p. 121, 6, 12. 13. saepe, ubi Euclides rectam aliquam vel punctum similiaque universe significaverat, Theon ad omnem dubitationem excludendam litteras, quibus in

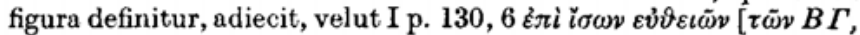

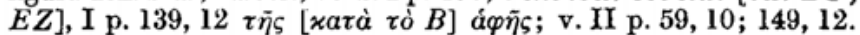
13; III p. 117, 3. 4; 188, 17; IV p. 120, 12; 121, 7, 14; 131, 3; 151, $16 ; 154,21$; cf. I p. 74,$15 ; 79,12$; II p. 92,$8 ; 113,20 ; 145,5,13$; 167,$23 ; 195,19 ; 220,10 ; 221,1$. de I p. 46,14 ad Theonem referendo dubito propter $\mathbf{F}$ mg. aliter quoque, quod Euclides significaverat, accuratius definiendum putavit interdum cum quadam significatione causae parvaque aliqua verborum mutatione, velut I p. 45, 4; 110, 16; II p. 178, 15; III p. 56, 2; cf. II p. $62,18$. $\sigma v v \vartheta \hat{\varepsilon} v \tau \iota$ addidit III p. $66,1, x a i \dot{\varepsilon} v a \lambda \lambda \dot{\alpha} \xi$ III p. 191, 9, 17. conferri potest etiam I p. 154, 20, ubi nune non dubito, quin scriptura codicis $\mathbf{P}$ unice vera sit. sed 1I p. 21,5 brevior forma orationis, quam habet $\mathbf{P}$, vix defendi potest.

Studio explicandi expoliendique, quae Euclides breviter neglegentiusque paullo lectorum iudicio confisus expresserat, haec

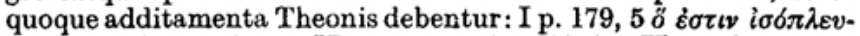

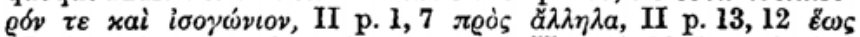

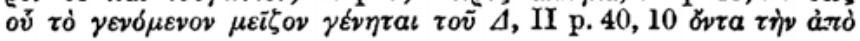

1) Hanc propositionem propositioni 17 libri VI respondere vo. luit, quia VI 16 in VII 19 de numeris repetitur; illam addidit ad similitudinem propositionis 23 libri V, quia plerasque propositiones libri $\mathrm{V}$ hic denuo de numeris demonstrari vidit; sed v. II p. 126 app. I ad 10. 11 . 


\section{PROLEGOMENA CRI'TICA}

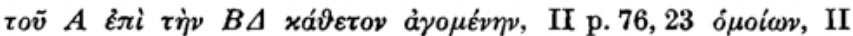

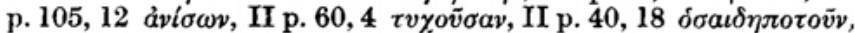

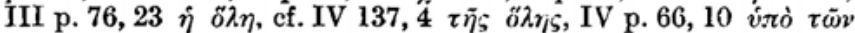

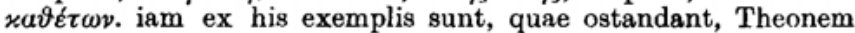
interdum in rebus mathematicis Euclidem additamentis suis supplere et corrigere voluisse, id guod magis etiam ex sequentibus

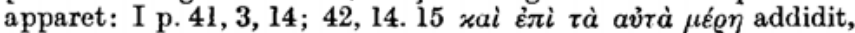

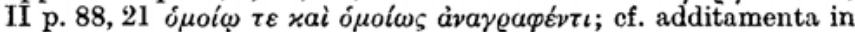
VI 28 p. $90,7-9$, de quibus v. p. 90 app. I ad 6 . eiusdem generis est, quod II p. 160,$14 ; 162,20 ; 164,21 ; 197,13$ interpolavit $\varepsilon \xi \bar{\eta}_{\varsigma}$ hic non magis necessarium quam $x a \tau \dot{\alpha}$ tò $\sigma v v \varepsilon \chi \varepsilon \xi \mathrm{V}$ deff. 9 - 10

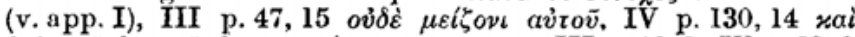

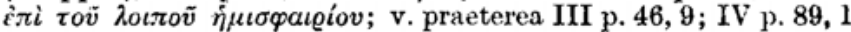

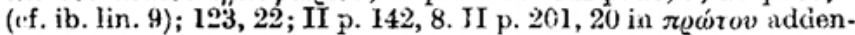

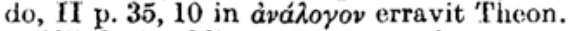

Aliis locis additamentr et supplementa magis ad orationis duri. tiam brevitatemque quandam tollendam spectant, velut cum in

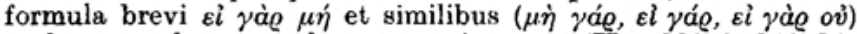
verbose supplet, quae eleganter omissa sunt (II p. 128, 9; 148, 14; 200,$11 ; 203,11 ; 222,13$; cf. II p. 138,$15 ; 157,1 \mathrm{sq} ; 214,3$ et locus aliquatenus similis in $\ddot{\eta}$ ov III p. 6,21 ; etiam III p. 104, 17 fortasse huc referri potest. ${ }^{1}$ )

Expositionem amplificavit II p. 117, 4; III p. 24, 20; 75, 8; cf. III p. 233, I5. 16.

Aiterum genus interpolationum est, ubi conclusio aliqua praevia et quasi gradus demonstrationis additur, velut I p. 101, 21. 22

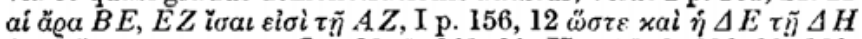

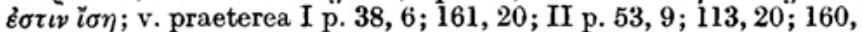
$8.9 ; 227,8$; III p. 5,$19 ; 85,6 ; 104,18.19 ; 160,1.2$. III p. 149 , 24 propter $\mathbf{F}$ mg. fortasse non Theoni tribuendum est additamentum his simile; I p. 137, 7. 8 in $\mathbf{P}$ error esse potest. quod magis etiam de II p. 66, 26 - p. 67, 1 dicendum, ubi ob constructionem et similitudinem membrorum error prope certus est. per $\tilde{\omega} \sigma \tau \varepsilon$, ut in exemplo secundo, conclusio interpolata inducitur II p. 78, 5; III p. 95,8 ; IV p. 87, 2, per $\delta \eta$ II p. 151,5 et in loco non prorsus simili II p. 91,7 . conferri possunt I p. 153,19 sq.; III p. 233,13 ,

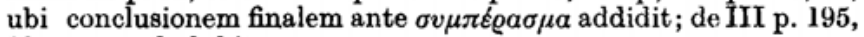
19 propter b dubito.

Tertium genus est amplificatio praemissorum membro intermedio interpolato, quod per $\delta \dot{\varepsilon}$ vel $\dot{\alpha} \lambda \lambda \dot{\alpha}$ infertur praemissisque ab Euclide datis annectitur, velut II p. 92, $8 \dot{\alpha} \lambda \lambda \dot{\alpha} \tau \dot{\partial} K M \tau \tilde{\omega} H B$

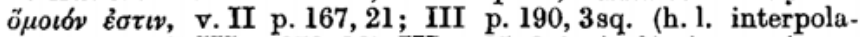

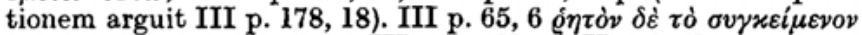
$\dot{\varepsilon} \varkappa \tau \tilde{\omega} v$ ảzò $\tau \tilde{\omega} v A B, B \Gamma$; v. III p. 87, 13; de II p. 84, 3 nunc propter $\mu \varepsilon \dot{\nu}$ dubito, III p. 62,17 propter $\mathbf{F}$ incertum est; praeterea hic etiam conclusio addita est, sicut etiam II p. 154, 2; III p. 92,3

1) Utrum Theon an librarii pro xai $\tau \dot{\alpha} \varepsilon \xi \tilde{\eta} \varsigma$ III p. 158, 16; 182, 19 verba propositionis reposuerint, non audeo decernere. 


\section{PROLEG OMENA CRITICA}

interpolatio paullo maior est. cum hoc genere etiam III p. 176, $21-$ p. 177,$\left.1 ; 190,8-10 ; 204,8^{1}\right)$ et interpolationes artificiosae I p. $85,8.9$; II p. 216,12 ; IV p. 143, 4-6 conferri possunt.

His exemplis postremis in quartum genus interpolationum traducimur varium et multiplex, quod continet causae indicationem a Theone additam. priori generi affines hi loci sunt: II p. 21, 7; 87,18 et interpolationes maiores II p. 117, $19-118,4$; III p. 200, 1-4; IV p. 74, 17; cf. etiam I p. 171, 3 sq.; II p. 148, 9, ubi causa per $\dot{\varepsilon} \pi \varepsilon i$ infertur. saepius tamen postea adiicitur per $\gamma \dot{\alpha} \rho$ annexa, velut I p. 147, 18; III p. 29, 7; 58, 9; IV p. 119, 24; 122, 24; 157,3;

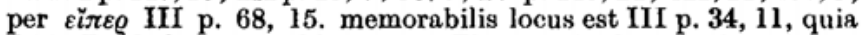
ibi in $\mathbf{P}$ scholium est additamento Theonis simile; cum ipsa forma $(\delta ı \dot{a} \tau \dot{o} \ldots$. ef. III p. 117,3. ad hoe genus etiam I p. 155, 1sq. referri potest.

Sequuntur additamenta minora, quae fere intra unum vel paucissima vocabula consistunt perspicuitatis vel concinnitatis causa adiecta. quo in genere multa necessario dubia sunt, neque omnia Theoni tribuenda esse affirmaverim, sed in multis vocabulis additis exemplorum copia tanta est, ut Theonis manum hic saltim agnoscere cogamur. unde in ceteris quoque huius generis interpolationibus suspicio oritur, Theonem ne in is quidem culpa liberandum.

In codicibus igitur Theoninis additum invenimus $\alpha \dot{v} \tau o \bar{i}_{\zeta}$ in

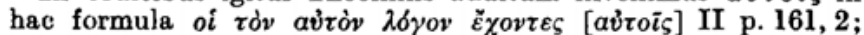
161, 27; 201, 16; III p. 233, 3 (et re vera saepissime ab Euclide additum est, velut in ipsa prop. VII 33, ne plura; sed necessarium non est; II p. 152, 5; 208, 7; 209, 5; 226, 16 in Theoninis bonis omittitur cum P, III p. 234, 1 contra P). practerea addidit av $\tilde{\omega} \tilde{\omega} v$

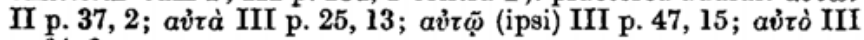
p. 64,9 .

$\tau \varrho$ ' $\gamma \omega v o v$ I p. 54,$12 ;$ II p. 43,$8 ; 49,12 ; 55,22 ; 65,6 ; 75,10$; IV p. 86, 12; 90, 12; 91, 26, 28.

$\tau \varepsilon \tau \varrho a ́ \gamma \omega \nu \circ v$ I p. 86, 9, 11; III p. 14, 9sq.; 15, 5, 8; 120, 6; 136,$17 ; 148,16 ;$ IV p. 82,25 et fortasse I p. 83,1 .

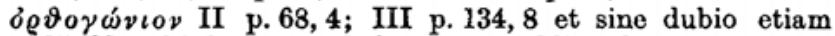

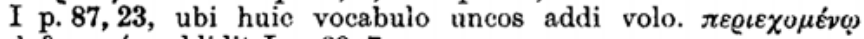
$\delta \varrho \vartheta \sigma \gamma \omega v i ́ \omega$ addidit I p. 89, 7.

$\pi a \varrho \alpha \lambda \lambda \eta \lambda \delta \gamma \varrho \alpha \mu \mu \circ \nu$ II p. 85,$3 ; 88,17 ; 89,3$; IV p. 57,7 ; $58,9$.

$\mu \varepsilon \gamma \varepsilon \vartheta o_{\text {II }}$ p. 22,19 ; III p. 2,$16 ; 6,8 ; 8,4$.

ả $\iota \vartheta \mu \grave{s}$ II p. 111,$11 ; 226,8$; III p. 10, 11, 14; 13, 16, 20 (bis); 14, 5 sq.; 233, 2 et sine dubio etiam II p. 109, 19; 122, 14; 124, 5 .

Hov $\alpha \varsigma$ II p. 165, 22 et sine dubio iam p.122, 14; nam diversitas generis $(\hat{\eta} A-\tau \dot{\nu} \nu \Delta)$ sufficit ad errorem evitandum.

$\sigma \eta \mu \varepsilon i o v$ III p. 76, 17; 170, 23; IV p. 119, 1 .

1) Cum hoc loco ef. additamentum Theonis III p. 160, 9 . 


\section{PROLEGOMENA CRITICA}

$\pi \lambda \varepsilon v \varrho \dot{a}$ II p. 49,14 et fortasse etiam II p. 175, 18; $\tau \tilde{\omega} v \pi \lambda \varepsilon v \varrho \tilde{\omega} v$ II p. 60,8 .

$\sigma \tau \varepsilon \rho \varepsilon \dot{v} v$ IV p. 54, 2; 118, 21.

$x v ́ x \lambda o v$ I p. 94,$22 ; 95,12$.

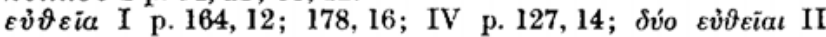
p. $59,7$. 19.

$\mu \dot{\eta} x \varepsilon \iota$ III p. 22, 233 «q ; $27,6,7 ; 29,7,13,19 ; 85,3 ; 144,7 ; 198$,

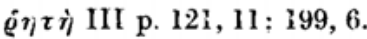

'wvia I p. 121, 23 (ef. p. 122, 18);140,18;144, 15;153,9; 170,$6 ;$ IV p. 31,1 .

Ftiam $\chi \omega \varrho i o v$ certum est additamentum III p. 194, 10, ubi etiam ordinem verborum mutavit Theon. magis dubir sunt $\mu \varepsilon \varepsilon \varepsilon \varepsilon$ III

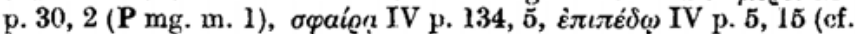

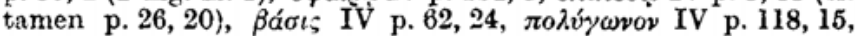

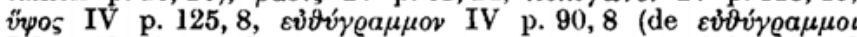
IV p. 69,19 , quod prorsus supervacuum est, non dubito); sed similitudo multorum locorum ex iis, quos supra attuli certiores, facit, ut veri simile sit, haec quoque vocabula a Theone addita

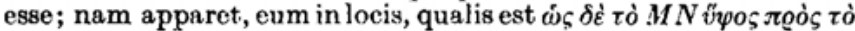
$\Pi N$, concinnitatis causa substantivum etiam altero loco ponere praetulisse (cf. verbi causa II p. 175, 18; III p. 14, 9, 12, 24; 15, 2 al., sed v. IV p. $58,13,22,24,25 ; 59,1$ al.).

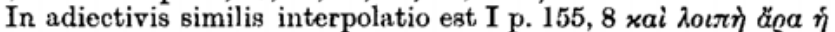
víò $M \Lambda N\left[\lambda_{o \iota} \tilde{\eta}\right]$ ] $\tilde{\eta}$ vंлò $\times \tau \lambda$.; item IV p. 35, 8. alterum necessarium non esse, apparet ex II p. 69,$11 ; 73$, 19; III p. 200, 1. etiam

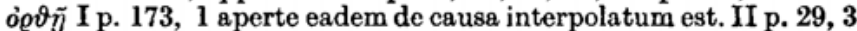

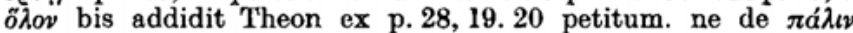
quidem interpolato I p. 142, 5; 158, 16; III p. 136, 11 dubito. v.

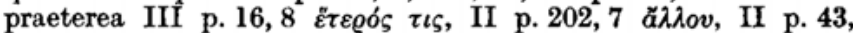

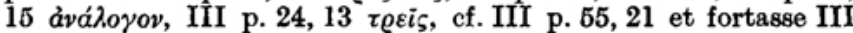

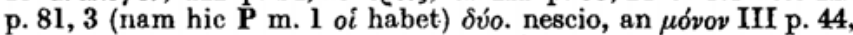
$3 ; 53,4$ omitti possit; III p. 134, 13 in loco prorsus diverso videtur a Theone additum esse; neque enim propter p. 135, 12 necessarium est. $\delta \circ \vartheta \varepsilon \iota \sigma \tilde{\omega} \nu$ IV p. 4, 17 prorsus inutile est et sine dubio Theoni debetur. idem ut orationem planiorem redderet, inutiliter

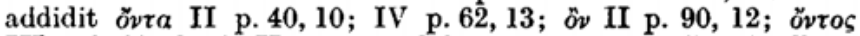
III p. 3, 23 (de $\hat{\omega} v$ II p. 109, 4 dubito; nam post $\mu \varepsilon \zeta \zeta \omega v$ facillime excidere potuit in $\mathbf{P}$ ); $\tau v \gamma \chi a ́ v o v \tau a$ IV p. 92 , 15; $\sigma v \gamma x \varepsilon(\mu \varepsilon v o v$

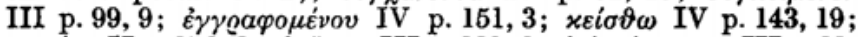

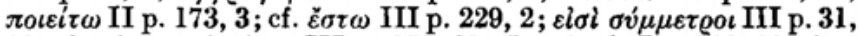

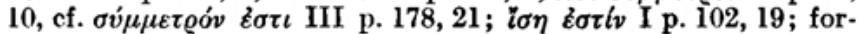

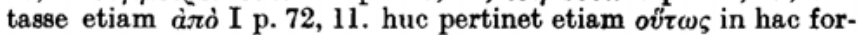

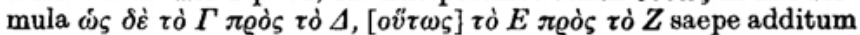
II p. 25,$3 ; 30,17 ; 49,15 ; 151,5,13 ; 159,8 ; 176,6 ; 193,8$; III p. 20,$20 ; 42,18 ; 206,17 ; 214,6$ (casui debetur, quod oṽ $\omega \varsigma$ II p. $34,15,16,17 ; 35,2,3 ; 151,21$ in omnibus Theoninis deest, quod quam facile fieri possit, ostendunt II p. 34,$14 ; 35,1,5$, ubi in melioribus deest). cf. omnino p. XLVIII. 


\section{PROLEGOMENA CRITICA}

Iam his exemplis ultimis ad minutias orationem spectantes perventum est, sed restant etiam minutiora.

Sexcenties addidit દo $\sigma \tau i$, imprimis post ă $\varrho$, sed item post alia vocabula velut $\mu \varepsilon i ́ \zeta \omega v, \mu \varepsilon ́ \varrho o \varsigma, \sigma v ́ \mu \mu \varepsilon \tau \varrho o \varsigma$ al., v. I p. 33, 22; 64, 8; 82,$6 ; 84,22 ; 116,13$; II p. 55,$19 ; 114,3 ; 226,13 ; 230,9$; III p. 36,$11 ; 57,18 ; 97,1 ; 98,4 ; 99,4$; III p. $89,20,23 ; 94,6 ; 100,8$; 103,$15 ; 133,18 ; 151,21 ; 174,9 ; 175,21 ; 178,18 ; 181,11,13$; 183,$24 ; 185,18 ; 186,5 ; 189,15 ; 190,13,14 ; 191,20 ; 195,2 ; 203$, $15 ; 206,7 ; 207,3 ; 208,4 ; 233,7 ; 234,9$; IV p. 13,$6 ; 54,8 ; 68,11$; $83.9 ; 92,12 ; 96,3 ; 97,7 ; 145,11 ; 188,18 ; 206,13$ (his duobus locis appendicis uncos omisi). etiam post ioos additur I p. 38, 13; III p. 160,20 ; IV p. 7,15 , sed multo saepius anteponitur ( $\varepsilon \sigma \tau \iota v$

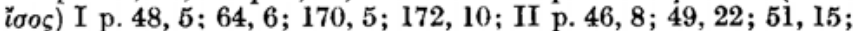

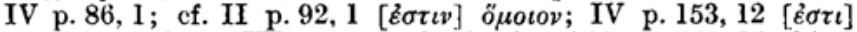

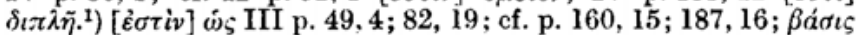

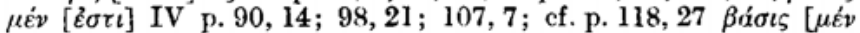
$\dot{\varepsilon} \sigma \tau \iota v]$. de III p. 97, 12; IV p. 158, 14 dubito, an errore in $\mathbf{P}$ omittatur $\varepsilon \sigma \tau i v$; I p. 170, 5 error manifestus est. $\varepsilon i \sigma i$ quoque satis frequenter a Theone additum est, v. I p. 97, 13; 162, 18; 163, 3; II p. 112,$1 ; 207,10$; III p. $16,1,22 ; 114,11 ; 200,6 ; 206,14$; IV p. 65,$25 ; 109,23$; aliquanto magis dubii loci sunt II p. 162, 8; 178,3 ; IV p. $92,15$.

In formula, qua ad demonstrationem transitur, $\lambda \hat{\varepsilon} \gamma \omega \delta \dot{\eta}$ vel nudum $\lambda \dot{\varepsilon} \gamma \omega$ habet Euclides; sed hoc Theoni displicuit, qui saepe $\delta \grave{\eta}$ addidit, velut II p. 12,$10 ; 183,14 ; 196,10,20$; III p. 99, 24; $161,6$; ef. $\delta \varepsilon เ x \tau \varepsilon \dot{\varepsilon}$ [ $\delta \dot{\eta}]$ III p. 108,$21 ; 110,7$; itaque etiam I p. 48,1 ; II p. 108,$20 ; 109,16 \delta \eta^{\prime}$ uncis includendum est; v. etiam I p. 176, 12; III p. 84, 3, ubi $\delta y^{\prime}$ in $\mathbf{P}$ supra scriptum est postea (v. p. XXXVI). quam facile interpolatum sit, apparet ex I p. 106, $13 ; 175,10 ;$ II p. 174,$8 ; 185,23 ; 221,3$; III p. 43,$1 ; 181,18$, ubi in compluribus codd. bonis omittitur. similiter ovv III p. 13, 15; 30, 11 a Theone interpolatum est (sed II p. 221, 1 vix omitti potest). ${ }^{2}$ )

In apodosi ăga saepe in Theoninis interpolatum est, velut I p. 59,3 (uncis notandum erat), II p. 177, 12;186, 1; 196, 12, 14; 215,8 ; III p. 64,$21 ; 79,21$ (prorsus similes sunt loci p. 87, 16; 152, 11 ; quare hoc quoque loco ăoc delendum); 130, 16;160, 10;181, 10 ; 195,$19 ;$ IV p. 10,$19 ; 129,14$. contra I p. 57,$17 ; 113,6$; II p. 111,$24 ;$ III p. 40,$17 ; 63,11 ; 67,6$ in Theoninis deest in

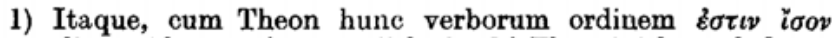
praetulisse videatur, fortasse ii loci, ubi Theonini hunc habent, P alterum, Theoni tribuendi sunt (I p. 60, 14; 80, 3; II p. 54, 4; $185,4$; III p. 116, 13, 14; 118, 19; IV p. 68,$19 ; 155,7)$. non dubitarem, nisi obstarent loci, ubi contrarium factum est, I p. 82, 9; 102,$19 ; 114,15$; III p. 108,$5 ; 177,19.20$; IV p. 23,$5 ; 36,1$; $128,4$.

2) Contra I p. 157, 16 ovv in $\mathbf{P}$ interpolatum est; fortasse etiam III p. 7, 12 delendum.

\section{LIV}




\section{PROLEGOMENA CRITICA}

apodosi, in $\mathbf{P}$ exstat, sine dubio interpolatum $\left.{ }^{1}\right)$, quare corrigatur II p. 111, 24.

$\mu \varepsilon \dot{\nu}$ II p. 192, 19 certissime Theoni debetur; respondet enim ver. bis $\tau o \dot{v} \delta \varepsilon \dot{\varepsilon} B \varkappa \tau$. ab eo pro lin. $20-$ p. 193, 5 substitutis. etiam IV p. 50,8 (uncis includatur); $118,8,11$ ei tribuendum videtur. quare veri non dissimile est, eundem Theonem hanc particulam etiam I p. 79,19 ; II p. 176,$8 ; 195,14$; III p. 58,$14 ; 119,20$; IV p. 59,2 ; 103,$26 ; 143,7$ interpolasse. nam Euclidem in talibus rebus non nimis religiosum sibique constantem fuisse, ut saepius iam observavimus, ostendunt loci I p. 91,$22 ; 152,12 ; 156,20$, ubi in for-

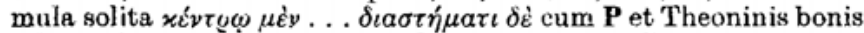
(ultimo loco omnibus) $\mu \varepsilon \dot{\nu}$ sublatum est. ${ }^{2}$ ) $\mu \varepsilon \dot{\nu}$ III p. 68,7 utrum a Theone additum sit an errore in $\mathbf{P}$ omissum, diiudicare non ausim.

Demonstrationem plerumque per $\gamma$ à $\varrho$ adiungit Euclides, sed interdum particulam omisit; Theon autem eam addidit II p. 66, 3; 180,$7 ; 218,1$; III p. 101,$18 ; 233,17$; IV p. 62,8 . fortasse etiam $o v v$, quod I p. 32,1 praebet $\mathbf{P}$ pro $\gamma \alpha \dot{g}$, defendi potest. I p. 17, 9;

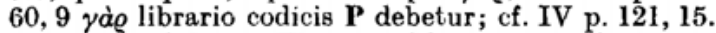

$\tau \iota \zeta$ sine dubio a Theone additum est in locis consimilibus II p. 130, 16; 131, 10. idem vocabulum II p. 109, 3 fortasse cum P omitti potest; nam comparatis p. 106, 12; 108, 3; 110, 1 intellegitur, cur Theoni in mentem venerit id concinnitatis causa

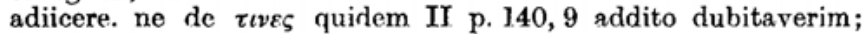
II p. 145, $2 \tau \iota v a$ in Pp omissum est.

Difficillima quaestio est de particulis $\tau \varepsilon$ et $x a i$, quia plerumque nihil prorsus interest, utrum ponantur necne. in iis ob inconstantiam codicum veri simile est maximam partem discrepantiarum, si non omnes, librariis imputandam esse. velut in formula $\delta \tilde{\tau} \varepsilon$

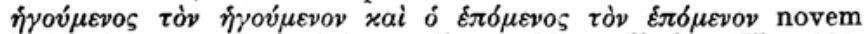
locis, si recte numeravi, in omnibus codd. est illud $\tau \varepsilon$ (II p. 128, $16 ; 132,1 ; 137,21 ; 150,2 ; 182,15 ; 200,24 ; 208,7 ; 209,5 ; 212,7$ ), II p. 162, 1 deest in $\mathbf{P}$ solo, II p. 155,10 in $\mathbf{P b q}$. itaque his duobus locis Theoni non debetur et sine dubio genuinum est. I p. 42, 13

1) Ceterum in hac particula vel addenda vel omittenda summa est inconstantia codicum. II p. 83,22 ; IV p. 135,17 cum P, I p. 61 , 11; II p. 170, 1 cum $\mathbf{P}$ aliisque codd. bonis omitti posse videtur (I p. 98, 2; 116, 9 alia correctio adhibenda est). sed I p. 53, 12; II p. 71,$19 ; 181,9 ;$ III p. 72,$18 ; 85,11 ; 110,5 ; 140,5 ; 146,22$; IV p. 15,$2 ; 138,10 ; 141,22 ; 149,6$ falso in $P$ omissum est, I p. 178 , $10 ; 183,9$; III p. 169,22 in $\mathbf{P}$ aliisque aeque falso, I p. 125, 11 ; II p. 148, 10; III p. 140, 3; 171, 23 in Theoninis bonis vel omnibus. I p. 38, 14; II p. 198, 21; III p. 91, 19; IV p. 31, 4, ubi omitti non potest, in $\mathbf{P}$ m. 1 supra scriptum est; idem factum est III p. 48, 22, ubi omitti poterat.

2) II p. 75, 9; III p. 142, $15 \mu \dot{\varepsilon} v$ in $\mathbf{P}$ manifesto errore additum est; quare etiam II p. 18,$13 ; 21,1 ; 36,3$; III p. 157,17 interpolatori deberi potest videri. II p. 151,$6 ; 168,21$; IV p. 130,17 res incerta, quia hic deficit $\mathbf{F}$. 


\section{PROLEGOMENA CRITICA}

P solus omisit; neque necessarium est; nam in loco simili p. 41. 11 etiam in melioribus Theoninis deest (p. 41, 7 in his solis, non in $\mathbf{P})$.

$\tau \varepsilon$ porro his locis in $\mathbf{P}$ solo deest et sine damno omitti potest I p. 50,$4 ; 55,2 ;$ II p. 151,$23 ; 156,3 ; 179,3 ; 195,20 ;$ IV p. 46, $\left.11 ; 85,17 ; 126,15 ; 154,2 ; 178,9^{1}\right) ; 184,2$. de I p. 78,7 dubito; nam $\tau \varepsilon$ a librario codicis $\mathbf{P}$ ad evitandam constructionem $\tau \varepsilon-$ $\mu \varepsilon \tau \dot{\alpha}$ insolitam illam quidem, sed bonam (Archim. III p. 350, 4), omissum esse potest; I p. 70, 8 in eadem constructione $\tau \varepsilon$ omittunt P p. contra I p. 137, 12, 14; II p. 69, 22; 233,2 ; IV p. $178,5 \tau \varepsilon$ cum Theoninis delendum videtur.

xai his locis a Theone interpolatum esse potest (ubi interpolatio certior videbatur, et ubi cod. F deficit, xai vel delevi vel uncis inclusi, in ceteris reliqui) I p. 41,$22 ; 136,16 ; 166,8 ; 167,2$; II p. 53 , $13 ; 57,8 ; 115,1 ; 116,8 ; 196,12 ; 220,3 ; 221,8$; III p. 16, 14;31, 5; 48,$20 ; 80,9 ; 87,13 ; 113,2 ; 114,5 ; 132,11 ; 134,3 ; 135,4 ; 209$. 13; IV p. 44,$20 ; 198,8$. at I p. 128 , 13; IV p. 176,18 xai errore in P nmissum, III p. 30,$11 ; 68,2 ; 158,3 ; 181,1 ; 205,18 ;$ IV p. 153, 21 errore additum, sicut II p. 166, 12, ubi librarius ipse correxit. ceteris quoque locis, ubi xai in $\mathbf{P}$ solo legitur, plerumque delendum existimaverim (I p. 61,$7 ; 114,16$; II p. 29,$21 ; 43,13 ; 49,17$; 141,$11 ; 151,18 ; 171,11$; III p. 71,$17 ; 130,3 ; 146,8 ; 225,4$; IV p. 121,$5 ; 134,3)$, quamquam est, ubi xai aegre cum Theoninis desideres, velut I p. 161, 20; III p. 5,8; ubi F non habemus, $x a i$ contra ceteros Theoninos retineri potest II p. 178, 20; 193, 5; IV p. 88,$1 ; 97,2 ; 124,10$ et fortasse etiam in locis gemellis II p. 180,$18 ; 183,8$.

Constat igitur, Theonem in eo vel praecipuam operam posuisse, ut amplificaret explicaretque, quae ab Euclide breviter dicta essent. quare per se non maxime est veri simile, eundem Theonem brevitatis studio adductum aliquando quaedam omisisse, nec omnino credibile esset, nisi certum quoddam genus omissionum in primis maxime libris tam saepe occurreret, ut casu factum esse vix credi possit. nam in expositione ( $\tilde{\varepsilon} x \theta \varepsilon \sigma \iota \varsigma$ quae vocatur), in qua Euclides plerumque omnes hypotheses propositionis repetit, multis locis in Theoninis codd. aliquid omissum est, velut I p. 8, $14 \tau \tilde{\eta}$

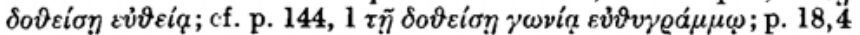

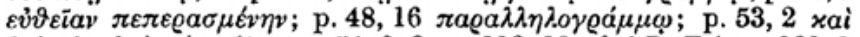

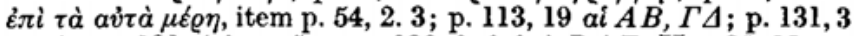

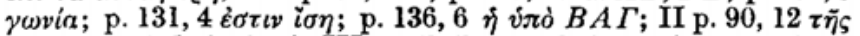
$A B$; p. 174,1 à $\varrho \vartheta \mu o l ;$ III p. 51,7 et p. $189,8 \mu \eta \dot{\eta} x \varepsilon$; p. 60,13

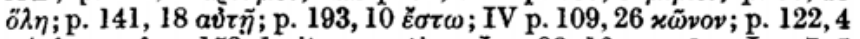
$x v ́ x \lambda \omega v$; cf. p. 152, 1. itaque etiam I p. 98, $16 \sigma \eta \mu \varepsilon i o v ;$ I p. 7, 5

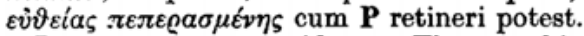

Iam cum constare videatur, Theonem hic diligentiam Euclidis tamquam nimiam improbasse et brevitati studuisse, videndum,

1) Cf. IV p. $186,7,9$, ubi $\tau \varepsilon$ in paene omnibus codd. omissum est. 


\section{PROLEGOMENA CRITICA}

ne alibi quoque Euclidis verba in breviorem formam redegisse sit existimandus.

I p. 53, 20. 21 igitur propter p. 53, 2; 54, 2.3 vix dubitari pot-

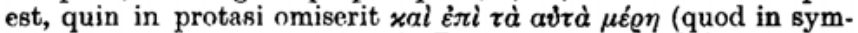
perasmate p. 54, 16. 17 etiam in $\mathbf{P}$ omittuntur, nihil demonstrat; cf. supra p. XLVIII; p. $52,18,19 ; 53,16.17$ propter $\mathbf{F}$ res dubia est). minus certum est, quod I p. 24, 16 et p. $25.25 \gamma \omega v i \tilde{\nu}$ in 'Theoninis omittitur; nam apud Proclum etiam deest. II p. 195, 23

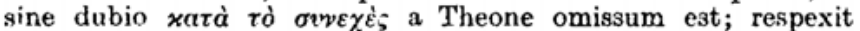
enim ad II p. 194, 5 (etiam in transponendis verbis $\varepsilon \xi \tilde{\eta} \varsigma \ldots$

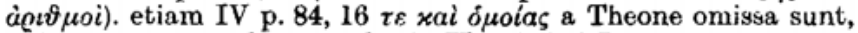
qui omnino totum locum refinxit. II p. 189, 3 P secutus sum, quia ibi Fnon habemus. cum locis supra p. XLVIII sq. allatis, ubi omisit, quae singulare aliquid haberent, conferri possunt I p. 47, 18; III p. $71,10,13.14 ; 129,14.15$ (cf. app. I ad 15);217, 5; 228, 19 (cf. 1. 4); IV p. 117, 22; 178, 9. 10 (IV p. 12, 6 adderem, nisi correctura in $\mathbf{B}$ a $\mathrm{m} .1$ esset).

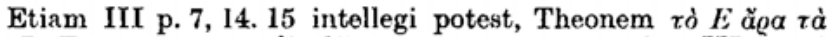
$A, B, \Gamma \mu \varepsilon \tau \varrho \varepsilon \grave{\imath}$ propter lin. 15 supervacua esse putavisse. III p. 5,4 ordine verborum offensus $\mu \varepsilon \dot{\gamma} \gamma \vartheta \hat{\vartheta}$ omisisse videtur; nam $\tau \dot{0} A B$

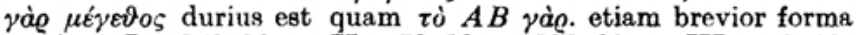
orationis I p. 159, 10 sq. ; II p. $79,13 \mathrm{sq} . ; 192,20$ sq.; III p. 69,19 ; 229, 17 editorem sapit, non librarium; cf. I p. 64, 1. 2; 117, 17; J29, 2. -- II p. $150,1.2$ (cf. p. 208, 7-9); 166, 22. 23; 168, 19. $20 ; 181,10 ; 182,9.10 ; 194,22 ; 202,19 ; 203,13-15 ; 206,13-15$; IV p. 97, 3; 198, $13 \mathbf{P}$ secutus sum, propterea quod in hac parte codice $F$ destituti sumus. incertiora sunt. quae $I$ p. 140, 1. 2; $170,14.15$; II p. 86,23 ; III p. $63,10.11 ; 195,12$; IV p. $71,1 \mathrm{e}$ solo $\mathbf{P}$ recepi.

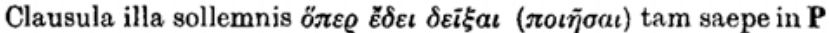
solo servata est, ut suspicari liceat, Theonem in ea omittenda neglegentiorem fuisse; v. I p. $22,4.5 ; 179,7$; 186, 5. 6; II p. 175, 5 ; 188, 20 (?); 219, 21 (?) ; 233, 2. 3; III p. 11, 15. 16; 22, 19; 35, 9; 42,$8 ; 45,16 ; 48,2 ; 58,15.16 ; 60,7.8 ; 61,10 ; 62,6 ; 64,11 ; 65$, $8 ; 67,12 ; 69,22 ; 70,17 ; 74,7 ; 79,4.5 ; 80,21.22 ; 83,4.5 ; 84$, $17.18 ; 86,12.13 ; 88,20.21 ; 97,12.13 ; 106,4.5 ; 109,5.6 ; 110$, 21. 22 et per totum fere librum X (praeter eos, quos citavi, XLIII locis). quominus haec omania pro certo Theoni imputem, prohibet librariorum in hac formula vel addenda vel omittenda inconstantia (saepe compendio significabatur potius quam scribebatur); nam interdum in PF solis servata est, saepe in omnibus codd. omissa vel saltim in pluribus melioribusque (v. I p. 113, 12. 13; II p. 172, 16. 17; III p. 18,$21 ; 49,16 ; 52,5 ; 53,18 ; 172,10 ; 177,11.12$ ), rarius falso adiecta (IV p. 54,$5 ; 63,8$ ). sed quod post corollaria fere in Theoninis omissa est, Theoni ipsi tribuere non dubito (v. II p. 76, 27; 108, 8; III p. 8, 13. 14; 209, 23; IV p. 69, 22. 23; 99, 13); nam videtur consulto a more Euclidis discessisse. ille enim, si testimonio codicis $\mathbf{P}$ confidimus, ubi corollarium propositioni subiunxit, plerumque clausulam illam in ipsius propo- 


\section{PROLEGOMENA CRITICA}

sitionis fine non posuit, sed eam post corollarium cum propositione cohaerens et quasi eius partem ultimo loco adiunxit (praeter locos allatos ef. III 1 et p. 95 app. I ad 16; III 16; VI 8, 19; XIII 16, 17; repugnant inter alia IV 15; VIII 2; XI 33.1) saepius ö $\varepsilon \delta \varepsilon \iota \quad \delta \varepsilon i \xi \alpha \iota$ etiam in fine propositionis ipsius interpolatum est. velut II p. 29,$17 ; 56,17 ; 71,25 ; 76,21 ; 108,4$; III p. 8,8 ; IV p. 69,16 alibi).

His omnibus perpensis sequitur, Theonem in Elementis edendis parum curasse, ut, quae Euclides re vera ipse scripsisset, e libris manuscriptis erueret restitueretque, eumque multo magis id spectasse, ut iis, qui ex Elementis mathematicam discerent, difficultates removendo explanandoque consuleret. quare editio eius cum editionibus grammaticorum Alexandrinorum comparanda non est, sed potius cum opera Eutocii in Apollonio edendo et cum interpolata recensione nonnullorum operum Archimedis ab homine Byzantino facta, de qua disputavi Neue Jahrbücher, Suppl. XI $384 \mathrm{sq}$. inter quos medium locum obtinet, illo inferior diligentia, hoc peritia mathematices longe superior. nobis, quorum hoc solum interest scire, quid Euclides scripserit, non probari operam Theonis longe alia voluntate susceptam, quis mirabitur? discipulis eius in Museo Alexandrino, quorum causa sine dubio editionem suam curavit - ibi enim saec. IV post Chr. n. mathematicam eum professum esse, testis est Suidas - non displicuit, et a posterioribus Graecis haec editio fere sola describebatur et lectitabatur, ita ut recensio antiquior uno tantum codice ad nostrum tempus servaretur. propter hunc favorem fortunae nobis congratulemur, Theoni hoc concedamus, vix meliora nobisque utiliora ab eo exspectari potuisse, quam quae praestitit, editionemque eius, si non ad Euclidis verba restituenda, at tamen ad studium peritiamque mathematices apud Alexandrinos quarti saeculi cognoscenda et ad rationem recensendi editorum antiquorum illustrandam plurimum conferre.

\section{Cap. III}

\section{De interpolationibus erroribusque ante Theonem ortis}

Ex iis, quae in cap. I disputavimus, apparet, scripturam codicis $\mathbf{P}$ et Theoninorum communem, si pauca fortuita excipias, talem nobis Euclidem praestare, qualis a Graecis saeculi quarti legeretur. sed tum iam plus annis sexcentis Elementa per manus librariorum mathematicorumque tradita erant. itaque certum est, verba Euclidis iam tum mendis inquinata fuisse. ea partim librariis partim interpolatoribus tribui possunt. quod ad librarios attinet, iam supra p. XXVII vidimus, quaedam Theonem fefellisse, quae a posterioribus in nonnullis codicibus emendata sunt. hic primum ad 3 .

1) In IV 5 et 16 singularis est ratio porismatis; cf. I p. 177 app. I 
errores aliquot afferemus, qui in omnibus codd. nostris reperiuntur nec fortuito orti esse possunt in utrisque, sed ad fontem communem Theone antiquiorem referendi sunt. eius generis est $\dot{\alpha} \tau \tau \dot{\varepsilon} \sigma \vartheta \omega$ pro éq $а л \tau \dot{\varepsilon} \sigma \vartheta \omega \mathrm{I}$ p. 122, 1, quam distinctionem Theon non curavit

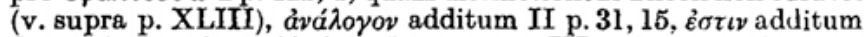
I p. 106, 18 (cf. p. 102, 21), äv omissum III p. 233, 5, xai vixò $\tau \dot{o}$ av่นò $v$ uo IV p. 50,4,10.11 omissum; aliquanto gravior error est I p. 105, 7 et in III 8 (v. vol. I p. 104 app. I ad 16). alibi fortasse non error librarii, sed neglegentia quaedam Euclidis in culpa est; v. I p. 74 app. I ad 14. 15, p. 156 app. I ad 15, p. 158 app. I ad 10, p. 178 app. I ad 19, II p. 74 app. I ad 9, p. 84 app. I ad 18. 19 , p. 154 app. I ad 6, p. 169 app. I ad 17. 18, p. 195 app. I ad

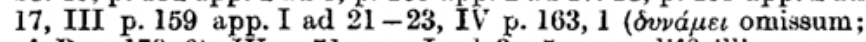
cf. $\mathbf{P}$ p. 179,6), IV p. 71 app. I ad 3-5. nam difficillimum est diiudicatu, quid Euclides hoc in genere committere potuerit, quid non potuerit, et contra consensum codicum tam bonorum cautissime adhibenda est coniectura, ubicumque non intellegitur, qua causa motus librarius aliquis rectam scripturam vitiare sustinuerit. itaque rationem Simsoni (Euclidis elementorum libri priores sex item undecimus et duodecimus ex versione latina Federici Commandini sublatis iis quibus olim libri hi a Theone aliisve vitiati sunt et quibusdam Euclidis demonstrationibus restitutis. Glasguae 1756,4 to) non probo; nam quamquam plerumque - nam ne hic quidem semper - suo iure errores ad mathematicam spectantes notat, in eo errare mihi quidem videtur, quod hos omnes Theoni aliisque irterpolatoribus tribuit; neque enim licet Euciidem ,ab omni naevo vindicare", ut verbis Hieronymi Saccherii (Euclides ab omni naevo vindicatus. Mediolani 1733) utar. velut p. 376

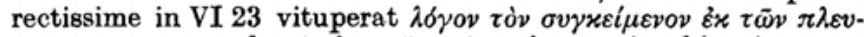
$\varrho \tilde{\omega} v$ pro eo, quod est $\dot{\varepsilon} \kappa \tau \tilde{\omega} v \tau \tilde{\omega} \nu \pi \lambda . \varepsilon v \varrho \tilde{\omega} v$ (Bc. $\lambda \sigma \hat{\gamma} \omega \nu)$, neque tamen dubito, quin ita scripserit Euclides (cf. II p. 81 app. I ad 18). fortasse etiam III p. 13, 19. 20 defendi potest scriptura codi-

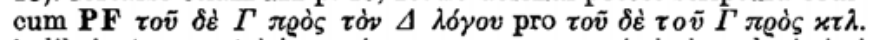
in libris stereometricis maxime errores nec paucissimi nec levissimi agnoscendi sunt, quos eo minus Euclidi tribuere dubito, quod haec pars geometriae tum demum diligentius pertractari coepta erat (v. XI 21, 24, 26, de qua ef. IV p. 44 app. I ad 8; XII 17, cf. IV p. 133 app. I ad l-3). ceterum Simsonus saepe recte acuteque verum vidit, et interdum obiectiones eius auctoritate codicis $\mathbf{P}$ confirmatae sunt, velut in VI def. 5 (v. II p. 39 app. I ad 12. 13).

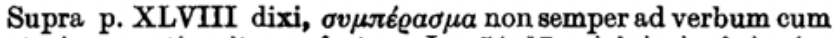

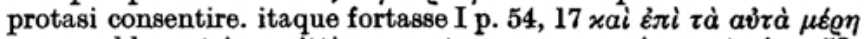
cum codd. nostris omitti possunt, quamquam in protasi p. 53, 20.21 in $\mathbf{P}$ aliisque exstant. ne hoc quidem negari posse videtur,

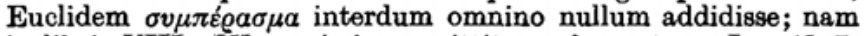
in libris VIII-IX saepissime omittitur: cf. praeterea I p. 43, 7. $8 ; 171,17.18$; II p. 78, 5-7; 110,3. itaque hoc quoque concedendum, Euclidem aliquando in conclusionibus longioribus pro verbis propositionis posuisse breviter xai $\tau \dot{\alpha} \varepsilon \xi \tilde{\eta}_{5}$; nam inde a 


\section{PROLEGOMENA CRITICA}

libro $\mathrm{X}$ hoc tam saepe et tanto inter omnes codices consensu fit, ut ante Theonem factum esse necesse sit, nec, si verum quaerimus, ulla est causa, cur ipsi Euclidi hane brevitatem abiudicemus. quare etiam I p. 98, 7 -10;103, 4-9; 107, 1-12;148, 21 - 149,3; $150,11-17$ codicum consensui nunc obtemperandum esse puto. ubi vero unus vel pauci tantum codices formam breviorem habent, plenior retinenda est (velut II p. 36, 25-28; 38, 4-6; 70,9-11; IV p. 8, 21-23); nam in talibus rebus arbitrium et consuetudo librariorum imperat, velut a IV p. 16, $11-13$ in V solo xal $\tau \dot{\alpha} \varepsilon \xi \bar{\eta} \varsigma$ saepissime occurrit (p. 18, 23. 24; 24,1-3; 25, 12-14; 26, 24$26 ; 28,3-5 ; 29,15-17$ al., p. 109, 17. 18); I p. 99, 7.8 in Theoninis est xai $\tau \dot{\alpha} \dot{\varepsilon} \xi \tilde{\eta} \zeta$, contra III p. 43, 10. 11; 44, 12. 13 in $\mathbf{P}$ solo; III p. 19, 22 alii alio loco verba propositionis abrumpunt. ubi xai

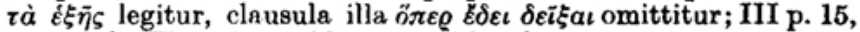
15 in solis Theoninis addita est. si hos locos excipias, ita raro in omnibus codicibus omittitur, ut dubitari possit, an semper resti. tuenda sit, praesertim cum in codd. saepe compendio scribatur, et omnino auctoritas eorum in hac re non magna sit (III p. 45, 16 in P servata est, in Theoninis omisea).

Erroribus ante Theonem ortis eos quoque locos, paucissimos sane, adnumero, ubi scriptura vera in uno solo Theoninorum servata est; ibi enim plerumque coniectando inventa esse putanda est, velut I p. 168, 2 (V, si collationi fides est); 178, 18 (p); II p. 13, 22 (V; cf. p. 153, 9) ; 88, 3 (V); 213, 7 (F); III p. 112, 6 (F); 166, 5 (V); 207, 11 (B); I p. 137, $14 \Gamma$, quod vix omitti potest (p. 138, I in $\mathbf{P}$ error esse videtur), in solo $\mathbf{F}$ m. 1 insertum est; cf. II p. 87 app. I ad 13. 14 (FV); II p. 111,24 error codicis $\mathbf{P}$ iam a Theone legebatur; nam inde orta est eius emendatio parum felix (cf. p. XXXIX). II p. 207,6 vero $ّ \sigma \tau \varepsilon$ fortasse cum codd. retineri potest mutata interpunctione, ita ut a $x a i$ lin. 9 apodosis ad $\dot{\varepsilon} \pi \varepsilon i$ lin. 5 incipiat. alii quoque loci hic praetermittendi sunt, quibus verba Euclidis communi mendo vitiata esse constat, num Theone id antiquius sit, non constat, quia error librariis in promptu erat, velut III p. 210, 2, 5, 19; cf. II p. 194 app. I ad 6.7 et III p. 75, 11. 12, ubi error fortuitus in $\mathbf{P}$ ex parte cum interpolatione certa (cf. supra p. LVI) Theoninorum conspirat. eiusdem generis est III p. 124, 4, ubi $\dot{\eta}$ delendum videtur, etsi tenent codices omnes (cf. III p. 124, 7; 125, 24); ut appareat inconstantia, ef. loci similes III p. 132, $2 ; 168,3,7 ; 170,7$, ubi $\dot{\eta}$ in solo $\mathbf{P}$ est (p. 133, 2 in $\mathbf{P B}$ ), p. 134, 10;171, 16, ubi $\mathbf{P}$ omisit, p. 169, 22, ubi omnes omittunt; denique p. 198, 22, 23; 230, $7 ; 231,8$. cf. omnino p. XXVIII sq.

I p. 4, 16 V, I p. $\tilde{5}, 6 \mathrm{~F}$ soli cum aliis fontibus antiquissimis consentire videntur, sed hi loci tam pauci sunt, ut nihil inde concludi possit.

Restat autem unum genus mendorum antiquorum, quod et latius patuit et manifestius coargui potest, in terpolationum.

Primum per se parum veri simile est, Euclidem duas demonstrationes unius propositionis dare voluisse, et haec dubitatio confirmatur, si naturam harum demonstrationum alterarum con- 


\section{PROLEGOMENA CRITICA}

sideramus. nam inter eas sunt, quae certissime ab Euclide profectae non esse demonstrari possint. velut quis credat, Euclidem ipsum demonstrationes receptas improbasse et postea novas breviores vel magis perspicuas addidisse, quas receptis praeferendas esse diserte significaret? sine dubio, si ita sentiret, eas recepisset, receptas omisisset. hac de causa damnandae demonstrationes no-

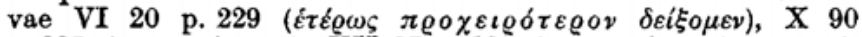

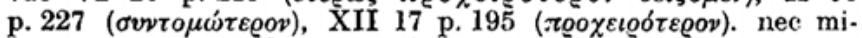
nus suspectae sunt demonstrationes norae in ipso contextu per $\hat{\eta}$ xai oür $\omega \varsigma, \hat{\eta}$ xai ă $\lambda \lambda \omega \varsigma$, similia moleste annexac, quales sunt III 7 p. 182, III 8 p. 183, ef. III 31 p. 185, X 32 lemma p. 222, XIII 18 p. 205. aliae nunc auctoritate codicis $P$, ubi postea in $\mathrm{mg}$. additae sunt, Theoni tributae sunt vel saltim ex verbis Euclidis ipsius renotae (II 4; VII 31 : X i, 6, 9, v. supra p. XXXVI). aliae rursus, ut X 105 et 106 (III append. $25-26$ ), et loco et vicinitate interpolationum manifestarum arguuntur; cf. X 115 p. 228 . haee omnia cum spuria esse constet, paucre etiam, quae restant demonstrationes alterae per se probae (III 9 p. 183 , III 10 p. 184, VI 30 p. 231 , VI 31 p. 232 , XI 22 p. 187), in suspicionem vocantur. nam facile intellegitur fieri potuisse, ut magistro vel editori alicui alia demonstratio in mentem veniret, quae ei magis placeret, sive iure sive iniuria, Euclidiana. documento sunt demonstrationes novae non in omnibus codicibus interpolatae, velut IX 22 p. 239 in F solo, XIII 5 p. 197 in $\mathbf{P}\left(\mathbf{b q}\right.$ q). ${ }^{1}$ ) etiam quae in codd. aliorum ope. rum mathematicorum Graecorum inveniuntur demonstrationes ulterae, si non omnes (nam in Archim. de sph. et cyl. II 8 genuina esse videri potest), at pleraeque suppositiciae sunt (de phaenomenis v. Studien p. 47 sq.); in Apollonio Eutocius nonnullas addidit (Neue Jahrbücher, Suppl. XI 361 8q.).

Eiusdem generis eae dilatationes demonstrationum sunt, ubi, cum Euclides more geometrarum antiquorum unum solum casum tractasset, interpolator reliquos addidit. et in XI 23 interpolatio manifestissima est (v. IV p. 37 app. I ad 7). in III 11 additamentum prorsus inutile est (I p. 185); in VI 27 p. 231, multis de causis suspectum (II p. 90 app. I ad 2), Theonis est.

In lemmatis quoque saepe est, cur dubitemus. non modo nonnulla a Theone (III p. $216 \mathrm{nr}$. 5) vel etiam post eum (in V ad X 27, $29,31,32,33,34$, v. III p. $218 \mathrm{sq}$.) interpolata sunt, sed etiam inter ea, quae omnes codices tuentur, non pauca suspecta sunt, maxime lemma ante $X 60$ p. 103, quo iam III p. 73, 2 tacite utitur (III p. 103 app. I ad 17). etiam de lemmate VI 22 satis, opinor, constat; nam per ambages demonstrat, quod e VI 20 statim concludi poterat, qua apertissime in re simili Euclides usus est VI 28 p. 92, 4 (v. II p. 92 app. I ad 5), ubi eadem occasio lemmatis

1) Quod Knochius, Untersuch. über die neuaufgef. Scholien des

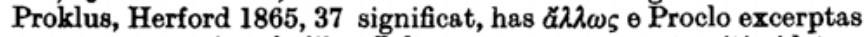
esse, errat; nec in solo libro I desunt, quo argumento niti videtur. longe antiquiores sunt. 


\section{PROLEGOMENA CRITICA}

fuerat. X 20 p. $217 \mathrm{nr} .7$ demonstrare conatur, quod in $\mathrm{X}$ def. 4 suppositum est; quod absurdum est. XII 4 p. 91 hoc in lemmate offendit, quod de altitudinibus in figura non ductis nec per litteras signatis disseritur; neque enim hoc moris est Euclidis; praeterea

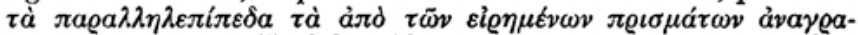

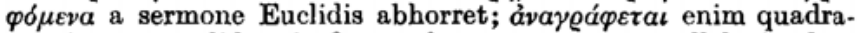
tum in recta, solidum in figura plana, nunquam parallelepipedum in prismate. de XIII 2 p. 140 v. app. I ad 8 (p. 140, 17 Euclides

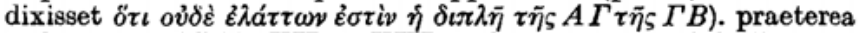
in lemmatis VI 22, XII 4, XIII 2 id quoque nonnihil offensioni est, quod ad propositiones praecedentes pertinent et postea ostendunt, quae in propositione usurpata sunt; eo enim ratio artificiosa, qua disciplina Elementorum exstructa est, turbatur et corrumpitur. eadem de causa suspecta sunt lemmata XI 23, XIII 13 (tum delendum IV p. 16l, $4 \hat{\omega} \zeta \varepsilon \xi \bar{\eta}_{\zeta} \delta \varepsilon \iota \chi \hat{\eta} \sigma \varepsilon \varepsilon \tau \alpha$, id quod discrepantiis codicum ad p. 161, 4 magno opere confirmatur), XIII 18, per se parum necessaria. sunt etiam inter haec lemmata, contra quae e scholiis antiquis documenta peti possint. ${ }^{1}$ ) lemma ad XII 2 quoque, quod iam Simsono p. 405 displicuit, supplementum demonstrationis antecedentis praebet; sed hoc deleto delendum etiam $\dot{\omega} \xi$

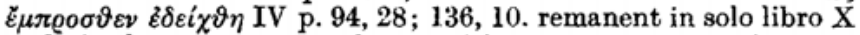
undecim lemmata, quae ad propositiones sequentes pertinent et impedimenta demonstrationes earum remorantia removent (cf. Proclus in Eucl. p. 211). eorum maxime lemma X 41 ob introduc-

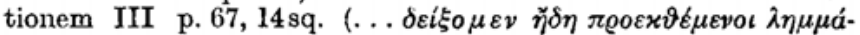

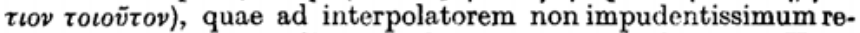
ferri non potest, aggredi non audeo, neque contra lemmata $\mathrm{X}$ 13, $16,21,32,53$ habeo quod dicam. duo lemmata post $\mathrm{X} 28$ dubia reddunt verba in fine alterius III p. 47, $17-48,2$ manifeste interpolata, sed fortasse haec sola delenda sunt. iudicium de lemmatis

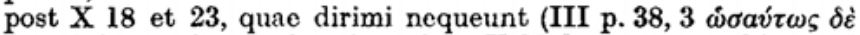

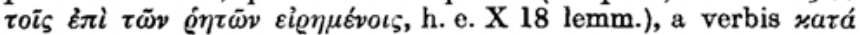

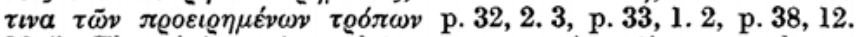
13 (in Theoninis om.) pendet. quae cum vix satis commode explicari possint, quia rectae longitudine commensurabiles esse supponuntur et ea de causa utroque modo (et longitudine et potentia) commensurabiles sunt, et ea et lemmata delenda sunt, cum praesertim lemmata cum additamentis aperte spuriis (III append.6 p. 216 et 8 p. $218^{2}$ ) coniuncta sint. lemma post X 9 una cum X 10

1) Ne hoc quidem practereundum est, quod is, qui glossema IV p. 162, 18 sq. addidit, sine dubio lemma XIII 13 p. 163 non habuit; tum enim additamento nihil opus erat. omnino contra haec lemmata afferri potest, Euclidem in libris stereometricis multa etiam difficiliora sine demonstratione assumere, v. verbi causa IV p. 131 app. I ad $19-23$.

2) Append. 8 in solis PV est (B m. 2) et ad append. 6 respicit (v. p. 218 app. I ad 1); append. 6 nomen Euclidis ne affectat qui$\operatorname{dem}(x a \lambda \varepsilon i$ p. 216, 10). 


\section{PROLEGOMENA CRITICA}

iudicandum est, quo pertinet, et in utroque tam multa sunt, quae offendant, ut vix retineri possint. nam primum X 10 nititur propositione sequenti (III p. 18, 15 sq.), quod Euclides nunquam commisit. deinde ne minima quidem causa est, cur commemorentur numeri plani non similes (III p. 17, 4 sq.; 18,3 ). denique $\dot{\varepsilon} \mu a ́-$ $\vartheta$

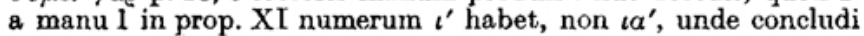
posse videtur, prop. $\mathrm{X}$ olim numero suo earuisse. removeatur igitur cum lemmate suo ab Elementis; nemo desiderabit.

Lemmatis interpolatis etiam propositio, quae vulgo est XI 38, adnumeranda est (IV append. 3 p. 192); est enim lemma ad XII 17 p. 129, 8. sed in b deest, et iam librarius codicis P libros ea carentes noverat (p. 192 app. II ad append. 3). itaque sine dubio delenda est, pratsertim cum loco prorsus pravo collocata sit nec omnino opus sit (v. Simsonus p. 404). paulio aliter res se habet in XIII 6 p. 144. hanc enim propositionem librarius codicis $\mathbf{P}$ (sive potius archetypi eius) in editione antiqua reperiebat, in plerisque autem exemplaribus editionis Theoninae deerat (IV p. 144 app. I ad 19); et deest in bq (q tamen in fine libri XII similem habet, sicut etiam pro scholio $V^{\mathrm{a}}$, v. IV append. 6 p. 196). nec dubitari potest, quin XIII 6, qualem recepimus, ab Euclide profecta non sit. nam primum in ipso $\mathbf{P}$ ad XIII 17 p. 180,10 scholium legitur, quod inutile eseet, si XIII 6 antecederet, quippe quod idem brevius ostendat. itaque cum hoc scholium scriberetur, XIII 6 nondum erat interpolata. hoc quoque suspectum est, demonstrationem alteram prop. 5 post XIII 6 in $\mathbf{P}$ coilocari; unde concludendum est, hanc demonstrationem (IV append. 7 p. 197) ante XIII 6 interpolatam esse - nam interpolatam eam esse, certum est et propter rationem universam demonstrationum alterarum (v. supra p. LXI) et quia praeter $\mathbf{P}$ solus $\mathbf{q}$ eam in textu habet (b $\mathrm{mg}$. m. 1, V m. l pro scholio) - et postea demum interposita prop. $6 \mathrm{a}$ propositione sua diremptam. etiam analyses propp. 1-5 in BV (et in P) post XIII 6 leguntur (IV p. 198 app. II ad append. 8), quia haec propositio post eas interpolata est; nam post prop. 5 locus iis est. deinde etiam ipsa propositio 6 suspecta est, quod in

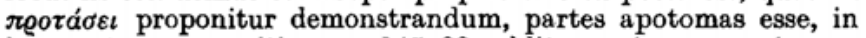
ipsa autem propositione p. 145,22 additur, minorem primam apotomen esse (in scholio illo codicis $\mathbf{P}$ haec offensio remota est p. 205, 7). ${ }^{1}$ ) itaque Theon recte fecit, quod XIII 6 non recepit, et fortasse tum nondum irrepserat in exemplar editionis antiquae, quo utebatur. postea igitur ex exemplaribus interpolatis illius editionis, quale exemplar fuit antigraphum codicis $\mathbf{P}$, etiam in nonnulla exemplaria editionis Theoninae (BV) transiit; tamen hoc quoque fieri potest, ut iam a Theone in editione antiqua in mg. inventa sit et eodem modo in editione eius collocata in aliis apo. graphis omissa, in aliis in textum recepta sit (cf. V q).

1) Omnino satis esset demonstrare, partem ma ior em apotomen esse; nam hoc utitur. 


\section{PROLEGOMENA CRITICA}

Damnata igitur XIII 6 videamus de XIII 17 p. 179, quo solo loco usurpari videtur. ibi enim p. 180, 10 sq. disertis verbis cita-

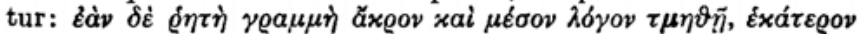

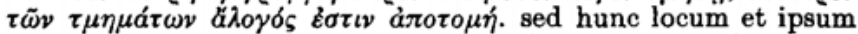
suspectum reddit usus prorsus non Euclidianus vocabuli $\gamma \varrho \alpha \mu \mu \eta े$ pro $\varepsilon \dot{v} \vartheta \varepsilon i a$ ( (v. I def. 4) ${ }^{1}$ ). et eodem loco p. 179, 22 olov - 180, 5

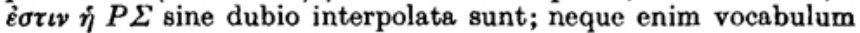
olov lin. 22 neque citata p. 179, 23-180, 1 propositio 15 libri $\mathrm{V}$ cum usu Euclidis convenit. itaque videndum, ne Euclides tantum p. 179, 16-22 $P \Sigma$, p. 180, 6-10 ‘ $\sigma \eta-x v ́ \beta o v, 12-13$ scripserit et lectori permiserit, ut ex XIII 1 concluderet, partem maiorem rectae rationalis secundum rationem extremam ac mediam sectae apotomen esse, quod neque difficile est neque in libris stereometricis incredibile (v. p. LXII not. l).

In corollariis de universo genere dubitari nequit; pleraque omnia et necessaria sunt et genuina iis tamen exceptis, ubi ipsi codices fluctuant, velut de coroll. I 15 p. $24^{2}$ ) omittendo non dubito, quamquam Proclus Psellusque id tuentur. etiam coroll. III 31 p. 138 et VI 20 cor. 2 aperte subditiva sunt (v. I p. 138 app. I ad 7. 8); sine dubio iam ante Theonem in $\mathrm{mg}$. addita erant. coroll. VII 3 p. 110 app. I ad 3 longe post Theonem interpolatum est ad lacunam demonstrationis II p. 140, 1 (v. app. I) supplendam. de corollariis a Theone interpolatis v. supra p. L. partes corollariorum interpolatae sunt I p. 159, 14-17 (v. app. I) et II p. 56, $18-57,3$; hic enim verba $x a i$ ह̌ $\tau \iota x \tau \lambda$. non habuit is, qui II p. 97, 4; III p. 54, 7sq.; IV p. 184, 8sq. hanc ipsam proportionem e VI 4, 8 demonstravit; itaque puto, ea iam ante Theonem in $\mathrm{mg}$. addita fuisse ab interpolatore aliquo et a Theone ibi relicta esse, sicut in $\mathbf{F}$ sunt; deinde corollarium et in $\mathbf{P}$ et in nonnullos Theoninos receptum est, in aliis ( $\mathbf{V}$ enim a $\mathrm{m} .2$ demum id habet) omissum (de porismatis in mg. scriptis cf. P XII 8 coroll.); P saltim öлre $\tilde{\varepsilon} \delta \varepsilon \iota \delta \varepsilon \bar{\xi} \xi a \iota$ p. $\tilde{7} 7,1$ servavit, quae verba et ipsa additamentum arguunt et ideo in Bp remota sunt. imprimis saepe in corollariis adumbratio demonstrationis ab interpolatoribus addita est, qui vererentur, ne statim appareret; at demonstrationem corollarii dare absurdum est (v. Proclus in Eucl. p. 301sq.). ea de causa deleo II p. 29, 18-22; p. 72, 5 - 7; III p. 15, 19 - 16, 13 (etiam aliis de causis suspecta, v. p. 15 app. I ad 19); p. 37, $22-38,2$ (obscura); IV p. 99, 9-12 (corrupta); delerem etiam IV p. 59,

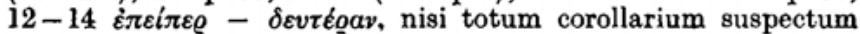
haberein, quod inutile est et in Fb omittitur (itrque si spurium est, idem in eo factum est, quod in VI 8 extr. p. 56). scholia explicationesque manifeste interpolata, quae plerumque ipsa forma (

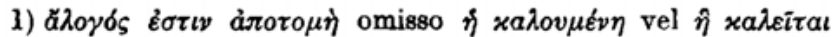
ferri potest; legitur enim in codd. bonis IV p. 157, 13; 180, 12.

2) Coroll. 2 ad I 15 in nonnullis codd. interpolatum est propter XI 23, ubi tacite usurpatur; v. IV p. 34 app. I ad 21.

\section{LXIV}




\section{PROLEGOMENA CRITICA}

21, 22; quod ex his in $\mathrm{V}$ omittuntur nr. 16, 17 et in mg. sunt $\mathrm{nr} .4$, $18,19,20,22$, eo confirmatur, eiusmodi additamenta primum (ante Theonem) in mg. scripta fuisse et Theonem ea eodem loco recepisse, unde postea in $\mathbf{P}$ nonnullisque Theoninis in textum irrepserint. ${ }^{1}$ ) eadem prorsus ratio est in magno illo additarento IV

append. nr. 8 p. $198-204$ (analyses et syntheses propp. $1-5$ libri XIII); nam hoc totum interpolatum esse, certissimum est, quippe quod toto genere a ratione institutoque Elementorum abhorreat. hoe quoque a principio in mg. fuit; neque enim aliter explicari potest, quo modo factum sit, ut prior tantum pars (v. IV p. 198 app. II ad append. 8) in textu sit in $\mathbf{V}$, reliqua in $\mathrm{mg}$. postea addita (quam libere in talibus appendicibus collocandis librarii versati sint, inde apparet, quod in codd. quibusdam analyses illae singulis propositionibus adiectse sunt). ceterum cum hic locus reliquiae antiquioris harum rerum expositionis analyticae, sive Theaeteti sive Eudoxi ea fuit, esse videatur (v. Pappus V 72 p. 410 ov̉ $\delta i \dot{a} \tau \tilde{\eta} \varsigma$

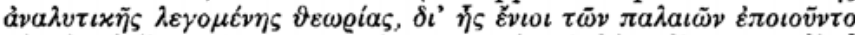

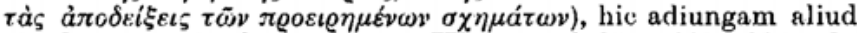
eiusdem generis glossema. nam III append. 27 p. $231-234$ subditivum esse constat, non solum quod inter additamenta manifesto spuria interponitur, sed etiam quia haec demonstratio post $X .9$ prorsus inutilis est, quippe quae casum quendam illius propositionis verbose ostendat. iam ex Aristotele (Anal. pr. I 23 et 44) cognoscimus, hanc ipsam demonstrationem ei notam fuisse, et veri similiter Hankelius (Beiträge zur Gesch. d. Math. p. 102) eam ad Pythagoreos ipsos huius doctrinae auctores rettulit. itaque hic quoque additamentum studio historico interpolatum deprehendimus.

Dixi supra, ut iam ab aliis intellectum est, velut a Gregorio p. 326, qui iure eo offensus est, quod scholii finis a sequentibus stereometricis pendet, etiam scholium illud III append. 28 p. $235-$ 236 in extremo libro $\mathrm{X}$ additum spurium esse, et hoc verum esse cum ex titulo ipso $\left(\sigma \chi \sigma \lambda_{i}(o v)\right.$ in $\mathbf{P}$ servato apparet, tum a scholiasta $\mathrm{X}$ nr. $\mathbf{l}$ disertis verbis confirmatur, qui in eo tantum errat, quod Theoni hanc interpolationem tribuit; multo enim antiquior est; et sine dubio hoc de suo ingenio prompsit; nam quod adiicit xai $\tau \iota v \varepsilon \varsigma a z \lambda \lambda o \iota$, ostendit, eum de auctore nihil certi compertum habuisse. mihi quidem satis veri simile videtur, hoc initium fundamentumque amplioris de irrationalibus disquisitionis ab Apollonio petitum esse, quem scimus de hac materia scripsisse.

Constat igitur, extremam partem libri $\mathbf{X}$ totam subditivam esse (III append. p. 227-236); quare cum Augusto eam in appendicem reieci. sed etiam de X $112-115$ dubito. neque enim usquam usui sunt, et cum X 111 aptissime ad finem perducta est dispu-

1) Cf. V 19 append. p. 229 et VI def. 5, quae Theon e mg. ed. antiquae recepit; v. praeterea p. LXIII-LXIV. eodem referendae seripturae Theonis III p. 34, 11; IV p. 95, 22 cum additamentis marginalibus codicis $\mathbf{P}$ congruentes. 


\section{PROLEGOMENA CRITICA}

tatio de irrationalibus XIII, quarum conspectum dat conclusio illa III p. 200-202, quae disputatio et per se omnibus numeris absoluta est optimeque distributa et ad analysin corporum solidorum regularium necessaria. utitur enim praeter X 73 (XIII 6, 11) etiam X 94 et 97 (XIII 11 et XIII 6), et cum his propositionibus opus ei esset, paucioribus defungi non potuit, quam quae dedit, nisi disciplinam abrumpere imperfectamque relinquere vellet; nam propp. 98-102 arte cum prop. 97 cohaerent, et propp. 103-111 quasi cumulum addunt toti doctrinae. propp. 112-115 contra neque cum reliqua disciplina irrationalium XIII Euclidianarum connexae sunt neque in libris stereometricis usurpantur. sunt quasi semina novae disciplinae subtiliorisque disputationis irrationales ipsas per se solas tractantis. itaque cum inter prop. 115 et scholium illud extremum similitudo quaedam sit, quippe quae genera irrationalium augeant, non dubito, quin hae quoque propositiones 112-115 e doctrina Apollonii promptae sint; nam antiquae sunt et bonac. hoc saltim constare putaverim, eas ab Euclide scriptas non esse.

Iam de reliquis interpolationum generibus videamus.

A certissima incipiamus. IV p. 68, 1-11 enim nemo dubitabit, quin interpolata sint ad explicandum illud $\delta \mu o i \omega \varsigma \delta \dot{\eta} \delta \varepsilon i \xi o \mu \varepsilon \nu$ p. 67, 23. optime Simson p. 403 ,verisimile enim est eam a quodam editore textui additam fuisse, ut ex verbis similiter demonstrabimus coniicere licet; ea enim non solent addi, nisi quando demonstratio non traditur". addi poterat, vocabulum ovi $\tau \omega \varsigma$ p. 68,1 prorsus insolito loco positum esse.

Eiusdem generis est IV p. 44, 9-20, quem locum miror Simsonum 1. c. non improbasse. nam primum absurdum est post verba $\delta \iota \dot{\alpha} \tau \grave{\alpha}$ av̉rà $\delta \grave{\eta}$ xai $\varkappa \tau \lambda$. p. 44, 8 demonstrationem ipsam addere; de iis enim idem prorsus valet, quod de ó $\mu$ oi $\omega \varsigma \delta \varepsilon i \xi o \mu \varepsilon v$ Simson

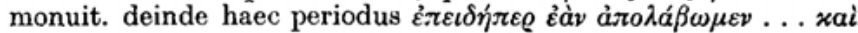

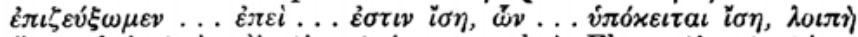
ã $\varrho x \tau \lambda$. iusto implicatior et sine exemplo in Elementis est. et iam ipsum vocabulum $\dot{\varepsilon} \pi \varepsilon \iota \delta \dot{r} \pi \varepsilon \varrho$ suspectım est (de Archimede cf. Neue Jahrb., Suppl. XIII 572). nam glossemata usitatissima, quae causae indicationem ab Euciide tamquam supervacuam perspicuamque omissam addunt, plerumque ab hac coniunctione incipiunt; v. II p. 93, 4, ubi etiam mentio parallelogrammi $H \Pi$ prava

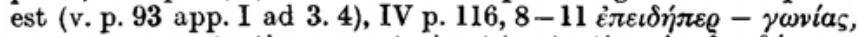

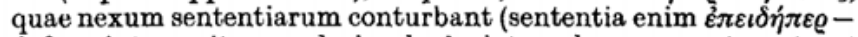
$\grave{\partial} \varrho \vartheta \tilde{\omega} \nu$ interposita apodosis ab $\dot{\varepsilon} \pi \varepsilon i$ tam longe remota est, ut

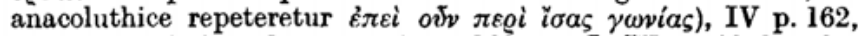
$2-5$, quae nimis verbosa sunt (v. p. 162 app. I), IV p. 162, 18-22, et ipsa supervacua et male cohaerentia ( lval 1.20 enim non habet, quo referatur). alius formae, sed generis eiusdem et aeque mani-

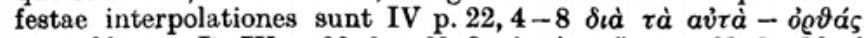

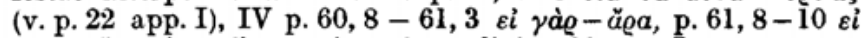
$\gamma \dot{\alpha} \varrho-\tau \sigma \alpha$, de quibus satis, opinor, dixi p. 60 app. I.

Interdum propositiones antea demonstratae falso repetitae sunt, 


\section{PROLEGOMENA CRITICA}

ubi usus earum est, id quod Euclides non facit, nisi ubi post longum spatium propositione aliqua utitur et eam in memoriam lectorum revocandam esse putat (velut VIII 8 in IX 1, VIII 20 in IX 2. VIII 23 in IX 3, VII 24 in IX 15). eius generis est II p. 206, 17-19 $\dot{\varepsilon} \dot{\alpha} \nu$ - $\dot{\varepsilon} \sigma \tau \iota v$ (VII 25); neque enim tales citationes postea per yào annectere solet, sed fere eas praemittit, velut boc ipso loco VII 24 (p. 206, 13 sq.); rursus III p. 55, 2-3 غ̇aे $\delta \dot{\varepsilon}-\mu \dot{\varepsilon} \sigma \omega \nu$ suspecta sunt, quia propositio simillima VI 17 bis tacite eodem loco

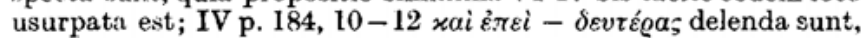
quia eadem definitione in proxime antecedentibus ter tacite utitur (p. 181, 8, 17, 24); cf. III p. 129 app. I ad 15.

Demonstrationem nembris intermediis interpositis explicare vo-

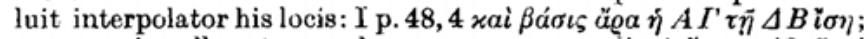
neque enimn ulla est causa bases commemorandi, et ǒ $а$ a p. 48, 5 , si haee verba retinentur, falsum est; I p. 116, 9 xai $\dot{\eta} A \Lambda \tau \tilde{\eta} \tilde{\zeta}_{5} M N$

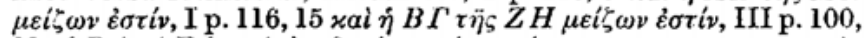

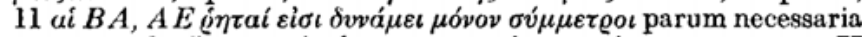
sunt et ob ăoa particulam necessarian omissam suspecta; II

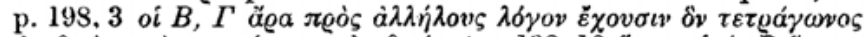

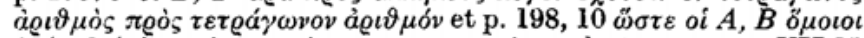

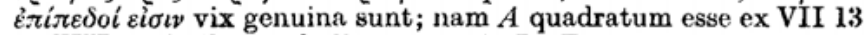
et VIII 24 facile concluditur, cum sit $B: \Gamma=A: B$, et $B, \Gamma$ qua. drati sint; et hoc modo in cubo ratiocinatur p. 198, 19-23;

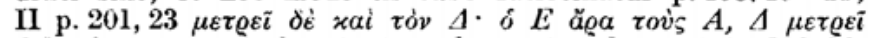
delenda sunt, quia hoc tantum demonstrandum est (p. 200, 10.

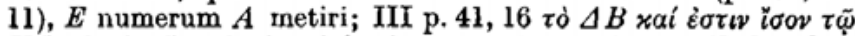

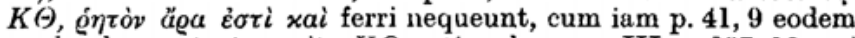
modo demonstratum sit, $K \Theta$ rationale esse; III p. 197, 18 xai

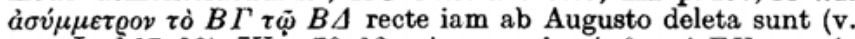

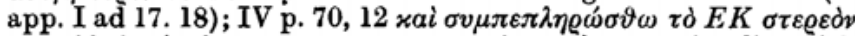
$\pi a \varrho \alpha \lambda . \lambda \eta \lambda \varepsilon \pi i \pi \varepsilon \delta \circ v$ suspecta sunt, quia moleste et insolito dirimunt, quae coniungenda sunt, $x \varepsilon i \sigma \vartheta \omega \tau \tilde{\eta} \mu \dot{\varepsilon} \nu B \ldots \tau \tilde{\eta} \delta \dot{\varepsilon} A \ldots$, nec in constructione talia omittere dubitat Euclides; IV p. 176 denique

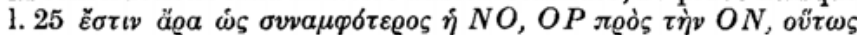

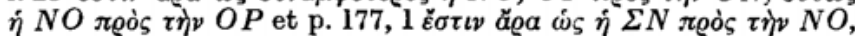

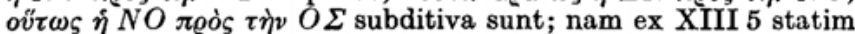
concludimus, $N \Sigma$ in $O$ secundum rationem extremam ac mediam sectam esse.

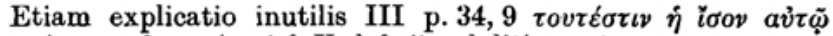

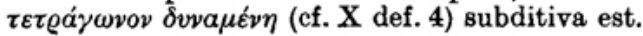

Minora sunt et fortasse librariis tribuenda ai et $\tau \dot{\alpha} \varsigma$ interpolata I p. 37, 16 et $a v i \tau \tilde{\omega} v$ aperte falsum II p. 87, 13 (om. FV, v. app.

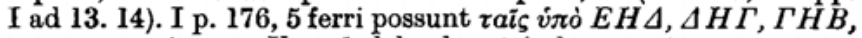
nec necessario cum $\mathbf{V}$ m. 1 delenda, etsi abesse poterant.

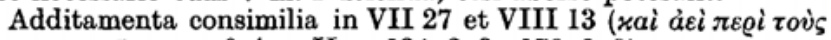

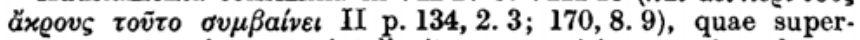
vacua sunt, quia per se intellegitur, propositionem etiam de numeris secundo et tertio loco productis valere et de quadratis cubisque demonstratam ad ceteras potentias transferri posse, su- 


\section{PROLEGOMENA CRITICA}

specta sunt, etiam quia ăx@o insolenter dictum est (p. 134 app. I ad 3), et quia ne verbo quidem in demonstrationibus comme. morantur. fortasse eidem interpolatori debentur II p. 2, 15-16 xai - vixáexn.

Interpolatorem non peritissimum geometriae fuisse, ostendunt

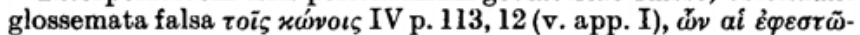

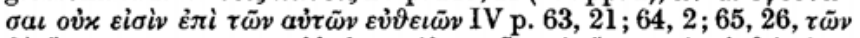

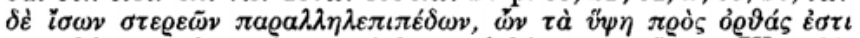

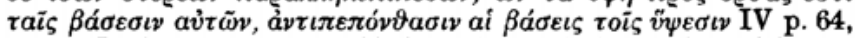

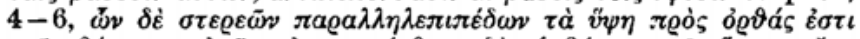

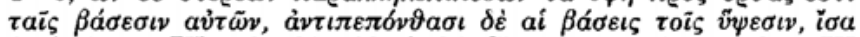

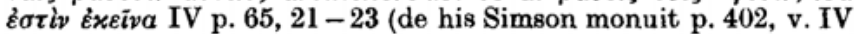
p. 63 app. I ad 19-21). sed cum in hac ipsa propositione XI 34

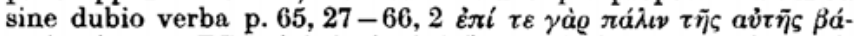

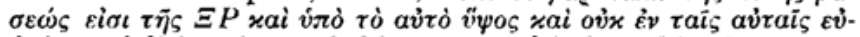
$\vartheta \varepsilon i ́ a \iota$ subditiva sint, quia hic quoque ultimis verbis ,,inepte excluditur casus alter", fortasse non solum verba supra notata, sed

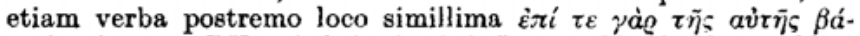

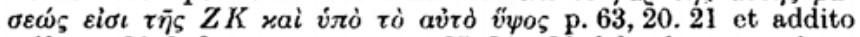
$\pi a ́ \lambda \iota v$ p. 64, 1. 2 praetereaque p. 65, 24-26 delenda sunt. cf. etiam de III 8 quae dixi I p. 104 app. I.

Haec sunt, quae ex ipsa re nullo auxilio extrinsecus petito nulli. que testimonio antiquo freti interpolata esse intellegere possimus. est tamen, ubi praeter locos iam allatos dubitari posse credam. velut in III 16 contra morem Euclidis (v. I 29; 32 al.) est, quod I p. 117, 9-11 non statim omnia, quae demonstranda sunt, commemorantur, sed primum tantum. itaque non sine causa de

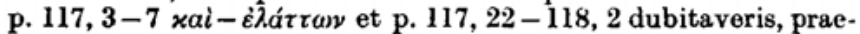
sertim cum per se suspecta sint (v. Simson p. 350); sed cum mos ille in III 7 et 8 non severe observatus sit, et constet, angulos mixtos iam antiquitus tractatos esse (Proclus in Euclid. p. 125sq.; cf. I def. 8-9; in catoptricis usui sunt), nihil certi affirmare audeo. eadem prorsus causa dubitandi est in III 31, ubi I p. 135, 18 -

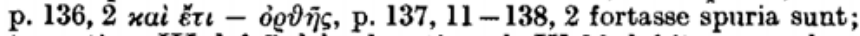
tum etiam III def. 7 delenda. etiam de IV 16 dubito, non solum quia de pentecaidecagono alibi non agit, sed etiam quia in sermone sunt, quae offendant (v. I p. 178 app. I ad 19 ${ }^{1}$ ). cf. tamen Proclus p. $269,11 \mathrm{sq}$.

Restant definitiones interpolatae. non dicam de VI def. 5; nam ea a Theone interpolata esse potest, quamquam in $\mathbf{P}$ a $\mathrm{m} .1 \mathrm{in} \mathrm{mg}$. additur (v. supra p. J.XV). sed in libro I constat, definitionem segmenti p. 3, 7 (v. app. crit. ad 7-8) interpolatam esse, quippe quae etiam III def. 6 loco aptiore legatur et a Proclo omittatur. etiam VI def. 2 cum Simsono reiicio (II p. 39 app. I ad 6-8), nec VII def. 10 retinendam esse puto (v. Studien p. 198 sq. ${ }^{2}$ ). praeterea

1) In his tamen $\dot{\gamma} \gamma \varepsilon \gamma \varrho a ́ \varphi \vartheta \omega$ p. 178, 4 (v. app. I) numerandum non est; v. enim IV p. 129, 5.

2) De deff. duabus libri V post Theonem interpolatisv.p. XXVI.

\section{LXVIII}




\section{PROLEGOMENA CRITICA}

de XI def. 11 valde dubito. priorem enim partem antiquiorem

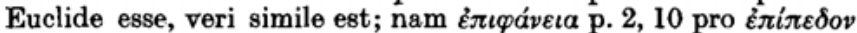
positum est more antiquiorum (v. Proclus p. 116, 17sq.). itaque fortasse Euclides ipse definitionem priorum $\sigma \tau o \iota \chi \varepsilon \iota \omega \tau \tilde{\omega} \nu$ servavit, praesertim cum definitio ipsius angulos solidos planis comprehensos solos comprehendat, antiqua autem etiam alia genera ( $ү \varrho a \mu$ $\mu \tilde{\omega} \nu$ et $\gamma o \alpha \mu \mu \alpha \bar{\zeta} \zeta$ p. $2,9,11$; utrumque genus diserte distinguit Hero def. 24). hoc quoque commemorandum, definitionem dubiam priore loco positam esse (nam si posterior dubia esset, non dubitarem, quin hoc quoque $\tilde{a} \lambda \lambda \omega \varsigma$ tollendum esset, sicut sine dubio II p. 3, 16-17 $\ddot{\eta}-\mu \varepsilon ́ \sigma \omega \nu)$. tamen non nego, mirum esse, Fuclidem duas definitiones dedisse vocabulo $a \ddot{a} \lambda \lambda \omega \varsigma$ alteri praemis-

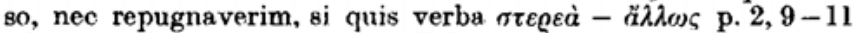
ad interpolationes supra p. LXV commemoratas referre voluerit.

De ceteris definitionibus maxime libri primi rectissime iudicat Paulus Tannery, vir doctissimus et de mathematicis Graecis optime meritus (sur l'authenticité des axiomes d'Euclide p. 7), Euclidern ex Elementis antiquioribus eas quoque admisisse, quarum

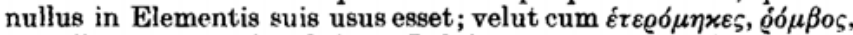

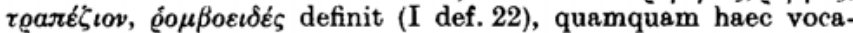
bula nunquam usurpavit; putavit enim, nec immerito, $\sigma \tau o \iota \chi \varepsilon i \omega \sigma t \nu$ vocabulorum quoque mathematicorum sibi dandam esse.

Aliam vero eiusdem viri docti sententiam non probo. putat enim, communes conceptiones (1 p. 5) omnes interpolatas esse (de postulatis quoque 4-5 dubitat, sed ipse argumentis suis non multum tribuere videtur, l. c. p. 11). sed cum constet (Proclus p. 194, 20sq.), iam Apollonium Pergaeum ${ }^{1}$ ) eas habuisse, interpolatio in tempus tam antiquum removetur, ut nihil dici possit veri dissimilius. quo modo, quaeso, factum est, ut Apollonium fugeret, axiomata, quae impugnaret, ab Euclide ipso profecta non esse? aut quid a mathematicis inter Euclidem et Apollonium committi potuit, quod non ipsi Euclidi imputare possimus?

Neque enim licet cum Tanneryio l. c. p. 11 interpolationem Apollonio posteriorem esse statuere, nisi verbis Procli l. c. vim afferre velis, nec quod de vocabulo xoıvai Ěvvoıa Stoicorum proprio dicit Tannery, magni momenti est. nam etiamsi concedamus - quod equidem nescio, quomodo diiudicari possit -, Euclidem ipsum vocabulum illud non novisse, tamen xoเvai Eี Evoı forma ab postulatis, quae omnia ab jंt $\sigma^{\prime} \vartheta \omega$ p. 4,14 pendent et infinitivum habent, satis manifesto distinguuntur (et revera Euclides titulum xoเvai Ëvvoı p. $193 \mathrm{sq}$. $\dot{\alpha} \xi \omega \dot{\omega} \mu \tau \tau a$ habeat). itaque nunc quoque - pace viri egregii dixerim - pro certo existimo, xotv. évv. 1-3 saltim ab

1) Hunc virum de primis mathematices fundamentis scripsisse

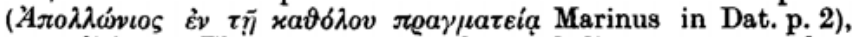
non editionem Elementorum emendatam dedisse, contra eundem Tanneryium disputavi in Philologi vol. XLIII 488sq. 


\section{PROLEGOMENA CRITICA}

Euclide ipso profectas esse. de xotv. Eัvv. 7-8 confiteor, aliquanto maiorem causam esse dubitandi, cum ab Herone omittantur et apud Capellam aliosque desiderentur. sed cum a Proclo, qui alias quasdam reiicit, sine suspicione legerentur, incerta est res. sed quicquid id est, hoc constat, xoเv. Ěvv. 9, quae in codd. nostris sedem sibi constantem nondum habeat, satis recenti tempore ${ }^{1}$ ) interpolatam esse, quia in I 4 p. 11, 15. 16 opus esse visum est. ne de xolv. Ěvv. 4, 5, 6 quidem dubitandum esse credo; neque enim video, qua ratione negari possit, Proclum eas in quibusdam fontibus non repperisse. cum harum interpolatione aliae connexae sunt. nam II p. 37, 21. 22 vix ambigi potest, quin in eiusmodi periodo

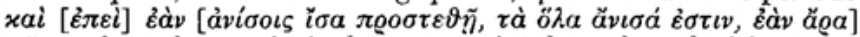
$\tau \tilde{\omega} \nu \quad \varkappa \tau \lambda$. verba uncis inclusa, quae intolerandam duritiam molestiamque sermonis habeant, delenda sint (nisi forte interpolatio peius etiam grassata est, v. p. 38 app. I), praesertim cum non ostendant, quod erat ostendendum. hoc si verum est, oritur suspicio etiam de locis similibus I p. $\tilde{1} 1,10.11 ; 52,13 \tau \dot{\alpha} \delta \dot{\varepsilon} \tau \tilde{\omega} v \underline{\imath} \sigma \omega v$

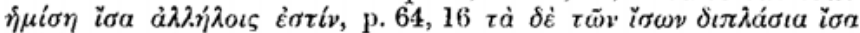

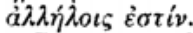

Itaque Euclides putandus est eas tantum xoเvd̀ દ̇vvoíaৎ recepisse, quae maxime essent necessariae, et quarum usus latius pateret; nam praeter receptas hic illic aliis utitur magis singularibus, velut iis, de quibus dixi II p. 135 app. I ad 15. 16, et in libro X $\tau \dot{o}$

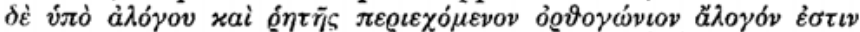
(prop. 38 p. $64,7.8 ; 41$ p. 67,$10 ; 75$ p. 130,15 al.) ${ }^{2}$ ).

Vidimus iam, etiam ex aliis auctoribus Elementa citantibus aliquando auxilium peti posse. colligamus igitur uno loco, quae eius modi passim in notis attulimus, et a Proclo teste praecipuo ${ }^{3}$ ) inci. piamus.

Constat igitur, Proclum vel codices vel alios fontes habuisse,

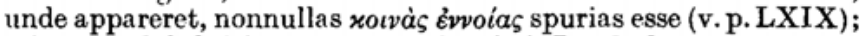
etiam quod definitionem segmenti omittit I p. 3, demonstrat, eum fontes puriores nobis habuisse. itaque non est, cur miremur, eum

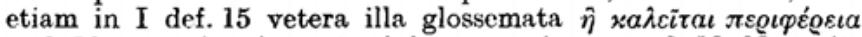

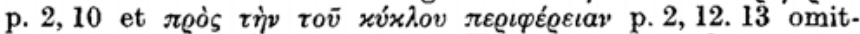
tere, sicut fontes alii antiquissimi, Hero (eum prius glossema ha-

1) Recentius etiam est, quod I p. 5 app. crit. ad 13 attuli axioma in $\mathbf{B}$ omissum, in $\mathbf{F b}$ in $\mathbf{m g}$. scriptum; in margine a Theone relictum et in $\mathbf{P}$ et in Theoninos aliquot irrepsit; cf. p. LXV.

2) Euclidem etiam alibi (nec in stereometricis tantum, de quibus v. p. LXII adn. 1) rebus non demonstratis uti, notavi II p. 190 app. I ad 22. 23, quacum conferri potest, quod etiam in X 5 p. 9, 11 usurpatur VII def. 20, quamquam in ea quoque de numeris, non de unitate agitur.

3) Hoc ita nec dico nec dixi, ut in verba Procli iurandum esse credam; sed praecipuum eum dico, quia nemo tot Elementorum locos citavit. quod Weissenbornii causa (Philol. Anz. XV 40 sq.) moneo. 


\section{PROLEGOMENA CRITICA}

buisse, non, ut antea putavi, ex Heronis def. 29 pro certo concludi potest), Taurus, Sextus Empiricus, alii. itaque etiam alibi, ubi omittit, quae omnes codices tenent, fortasse ei obtemperandum est, velut in $\dot{\alpha} \lambda \lambda \dot{\eta} \lambda a \iota \varsigma$ I p. $12,6,7 ; 13,23,24 ; 40,5 ; 41,7$, in $\varepsilon x \alpha-$

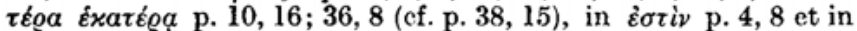
primis p. 6,4 , in $\delta v$ o p. $5,6(\mathrm{~F}) ; 29,11, \tau \rho \varepsilon \tilde{I}_{5}$ p. 44,9 , in xai p. 52 , $19 ; 53,21$. alibi rursus iniuria aliquid omisit, velut p. 3,16 (contra Heronem), p. 4l, 7 ; de p. $31,1-3$ dubito; p. 6,$3 ; 9,17$; 15, 24; 22,$8 ; 48,10$ non est, cur a scriptura codicum discedamus et scripturam Procli recipiamus neque per se neque ulla constantia discrepantiae commendabilem. si locos supra allatos excipias, quos fortasse ex aliquo fonte antiquo, velut commentario vetustiore, desumpsit Proclus, codex eius non optimus fuisse videtur; interdum enim cum deterioribus nostris coneentit (cum BV in errore aperto p. 2,3 , cum b p. 3,$15 ; 31,12$, cum $V 31,13 ; 40,5$; cf. p. $3,17,18$; $24,15)$ nec interpolatione in nostris non obvia prorsus libera videtur fuisse (v. p. 3,$\left.9 ; 22,8^{1}\right) ; 24,16 ; 36,5 ; 45,15 ; 58,11$ ); etiam (ie-

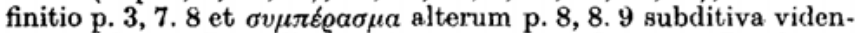
tur, et de corollario I 15 p. 24, 10-13, quod praeter Proclum e codd. nostris F solus in textu habet, prope certum est; qua de causa ne p. 36, 9 quidem scriptura Procli et codicis $\mathbf{F}$ communis recipienda. cum $\mathbf{P}$ solo tribus tantum locis consentit (p. $317 \delta \dot{\varepsilon}$

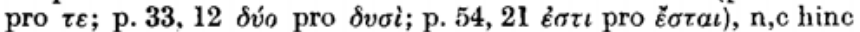
concludi potest, Proclum editione antiqua usum esse ${ }^{2}$ ) (euos in studiis Euclideis p. 185 attuli locos, nunc codicum scripturis plenius cognitis aliter se habere compertum est); cum $\mathbf{P}$ et quibusdam Theoninis conspirat p. 2,$2 ; 3,9 ; 5,7 ; 51,17,18 ; 52,18.19 ; 53$, 20.21, cum Theoninis contra $P$ aliosque fontes p. 24, 16. maxime memorabiles ii loci sunt, ubi Proclus cum uno et altero codicum

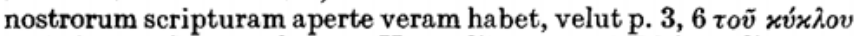
omittit cum b p (confirmant Hero aliique), p. 4, 16 in ordine verborum cum $\mathrm{V}$ et quibusdam fontibus antiquis consentit, p. 5, 6 cum $\mathbf{F} \delta \dot{o}$ omittit sicut alii fontes antiqui. de talibus locis cf. quae dixi p. LX. ${ }^{3}$ ) inter scripturas Procli proprias paucae praeferendae sunt, velut p. 21,$3 ; 22,9 ; 35,2 ; 44,9 \delta$ jo pro $\delta v \sigma i$ (cf.

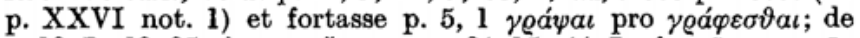

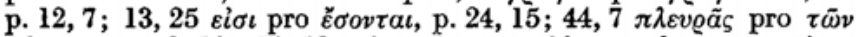
$\pi \lambda \varepsilon v \varrho \bar{\omega} v$, p. 3,$12 ; 10,13 \pi \lambda \varepsilon v \varrho \tilde{\omega} \nu$ pro $\varepsilon \dot{v} \vartheta \varepsilon \varepsilon \tilde{\omega} \nu$ ob constantiam dubitari potest, sed ultima saltim discrepantia interpolationem olet; etiam p. 23, 12 nostri codd. verum habere videntur. in adiaphoris codices nostros sequendos esse puto, cum consensus eorum tempore aetatem Procli superet (v. p. 3,$15 ; 4,5 ; 5,11 ; 6$, 3,$4 ; 35,3 ; 63,6$ (bis) et in ordine verborum p. 1, 13, 14; 4, 4, 10;

1) Hic scripturam codicum confirmat II p. 99, 13.

2) De I 13 p. 21, 2 nunc dubito.

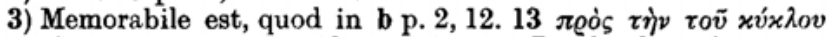
$\pi \varepsilon \varrho \iota \varphi \varepsilon \varepsilon \varepsilon ı \nu^{2}$ erasa sunt; videtur igitur ex Proclo aliove fonte antiquo correctus. 
$5,6,10 ; 10,12 ; 17,6 ; 29,9 ; 32,13 ; 33,13 ; 44,8$ (bis); p. $30,23.24$ alibi nostrorum codicurn ordinem, alibi proprium habet). sed negari nequit, eum hic illic vestigia scripturae integrioris servasse, sive eam e codice integriore sive ex aliis fontibus prompsit; cuius rei exempla hoc loco eo magis colligenda esse statui, quod in editione ibi tantum ei obtemperavi, ubi aliud accessit testimonium, et quod Weissenborn (Philol. Anz. XV 40sq.) Proclo omnem fidem abrogat.

De ceteris fontibus externis brevis esse possum (v. Studien p. 186 sq.). qui Theone antiquiores sunt, plerumque cum optimis codd. nostris consentiunt, velut Hero $^{1}$ ) I p. 2, 2; p. 94, 11; II p. 39, 7, et cum P solo II p. 1, 7; cf. I p. 93, 5 (etiam in libro V definitionem analogiae omittit); I p. 93, 8 fortuito cum $\mathbf{V}$ consentit; cf. etiam p. 93, 14 (p). quod I deff. $11-12, V$ deff. $6-7$ permutat et $\mathrm{V}$ def. 10 omittit, nullius momenti est ob genus totum definitionum Heronianarum (in libris X - XI liberius definitiones Euclidis ad suum usum transformat). magis memorabile est, eum def. 2 libri VI habere; sed v. II p. 39 app. I ad 6-8. interpolationes I p. 2, 12; 3, 6 non habet, nec credibile est, eum glossema eiusdem generis p. 2, 10 iam in Elementis legisse; nam I def. 15 formam genuinam habent auctores posteriores Taurus, Sextus Empiricus, Proclus. puto enim, Elementa saeculo fere tertio interpolatione maxime vitiata esse; nam Sextus Empiricus textum integrum habuit, Iamblichus contra interpolatum (v. Studien p. $197 \mathrm{sq}$.), sed sine dubio etiam exemplaria integriora diu in manibus hominum fuerunt, nec interpolatio omnia occupavit, sicut videmus, nostros codices interpolationibus a Iamblicho conmemoratis carere.

Auctores Theone posteriores nostris codicibus non antecellere, non est, quod miremur; de Ammonio (nunc Philop. Categ. cf. vol. I p. XLI) v. I p. 2, 2 (= Pbp); de Simplicio v. I p. 2, 2 $(=\mathrm{B} \mathrm{FV}) ; 94,11(=\mathrm{PB} \mathrm{p})$; II p. 58, $2(=\mathrm{P})$; I p. 4,16 cum Proclo et $\mathbf{V}$ consentit; de Olympiodoro v. I p. $36,9(=\mathbf{P B V}$ ) de Eutocio (se. Eutoc. in Archim. cf. vol. I p. XL) v. I p. 5, 13 (interpolationem habet); 30,24 (= PVbp); in III 8 meliorem scripturam habuisse videtur; sed VI def. 5 iam habet. de Philopono difficilis est quaestio; nam diversis codicibus usus esse videri potest, nisi locos, ubi integriorem scripturam habet, e fontibus antiquioribus transsumpsit. I def. 15 enim modo sine glossematis antiquis (in phys. b IIII), modo cum altero (et cum a mbo eodem tempore interpolata esse necesse sit, ipse prius omisisse putandus est) citat, I p. 2, 2 modo verborum ordinem deteriorum codicum,

1) Quamquam in definitionibus Heronianis, quae feruntur, sunt, quae interpolationes post Heronem factas arguant, tamen maxima ex parte eas ab Herone profectas esse puto; certe antiquae sunt. sed verba Euclidis plerumque tam libere reddunt, ut in rebus criticis non magni momenti sint. 


\section{PROLEGOMENA CRITICA}

modo meliorum (in phys. i IIII), I p. 2, 2 modo $i \sigma \omega \nu^{1}$ ) habet cum codd. (in anal. p. 65), modo omittit cum Ammonio aliisque (in phys. i IIII), I p. 21, 2 modo $\dot{\varepsilon} \dot{a} \nu$ habet cum Proclo, modo ó $\tau \alpha v$ cum P. I p. 4, 1; 6, 3; 22, 8 cum codd. contra Proclum conspirat; I p. $5,1,7$ cum codd. deterioribus, II p. 61,17 contra cum $\mathbf{P}$ consentit; interpolationes Theone antiquiores habet I p. 6, 5; II p. 40, 1-3; III p. 231 (nam append. 27 agnoscit Comm. in anal.pr. p. LXIsq., in anal. post. fol. $30^{\mathrm{v}}$, v. Studien p. $212 \mathrm{sq}$.). de interpolatione quadam in VII def. 8 in quibusdam codd. a diasceuasta Philoponi reperta v. II p. 103 app. I ad 13. 14.

In hac tota quaestione, quae est de locis Euclidianis apud posteriores citatis, hoc quoque loco iis contentus fui, quae in studiis Euclidianis collegeram; nam quas nunc habemus editiones plerorumque commentariorum Aristotelicorum, eae in rebus criticis neque habiles sunt nec satis fide dignae. si quando omnes illi commentarii ea diligentia editi erunt, qua Simplicii in libros de caelo. investigatio locorum Euclidianorum denuo facienda est et utilins faciliusque fieri poterit.

Apud Byzantinos viguisse studium Elementorurn, testimonio sunt et scholia Byzantina paene innumerabilia et codices permulti a saeculo nono ad decimum quartum in oriente scripti. hoc quoque commemorandum, in catalogis bibliothecarum maxime Constantinopolitanarum saeculi XV a Foerstero editis (de antiquitatibus et libris mss. Cnopolitanis. Rostoch. 1877) plures referri codices (in

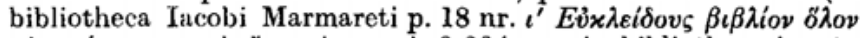

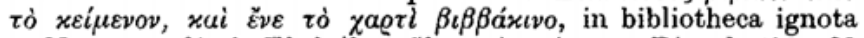

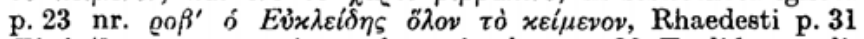

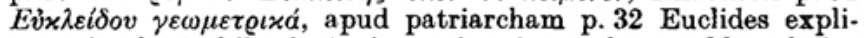
catus in charta bibacina), sicut etiam in catalogo codd. ecclesiae

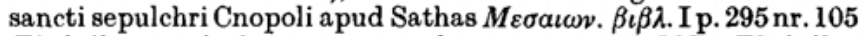

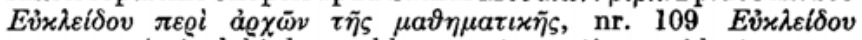

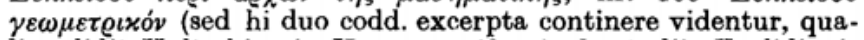
lia edidit Hultschius in Herone p. 41 sq.). de studiis Euclidianis saeculi undecimi testis est Psellus (Studien p. 172 et p. 213sq.; cf. quae ipse de mathematicis studiis suis narrat apud Sathas,

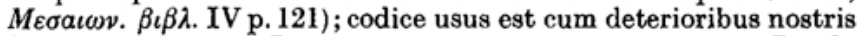
fere consentienti (v. I p. 2, 2; 3, 18; 4, 1; 21, 2; 24, 10; cum Proclo I p. 4, 10, cum Ammonio aliisque I p. 2, 2, cum Philopono II p. 72, 4 (cf. app. I), cum P solo IV p. 4, 3, cum P aliisque II p. 104, 19).

Saec. XIII - XIV studium mathematices subtilioris paene perierat, et vulgo intra geometriam planam et tritissima quaeque e doctrina numerorum stabant homines, ut apparet e loco prae-

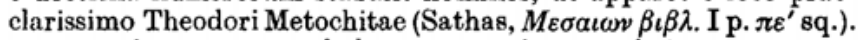
narrat enim, se, cum scholas grammaticorum rhetorumque percurrisset, post logicam Aristotelis ad mathematicam animum convertisse, sed frustra magistros doctrinae subtilioris quaesisse

1) Commemorandum tamen, ï $\sigma \omega \nu$ in $\mathbf{P}$ omissum esse I p. 95, 6, ubi haec def. citatur. 


\section{PROLEGOMENA CRITICA}

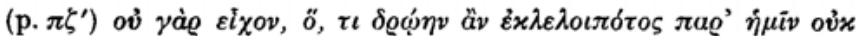

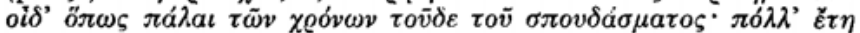

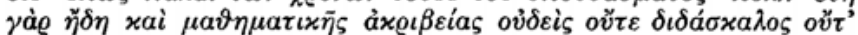

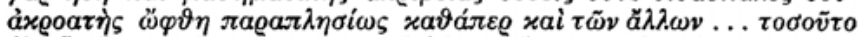

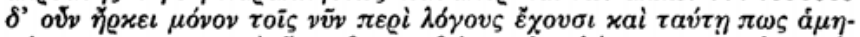

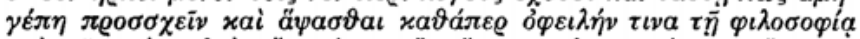

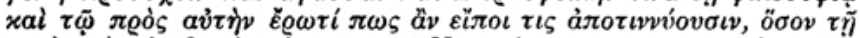

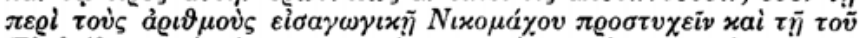

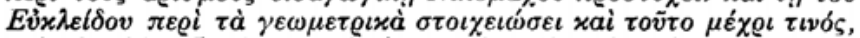

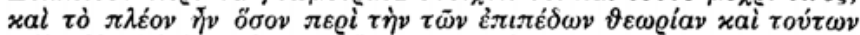

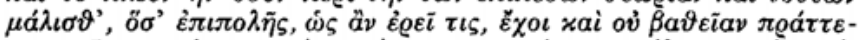

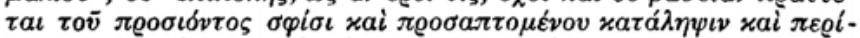

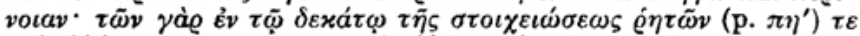

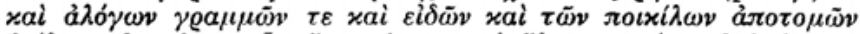

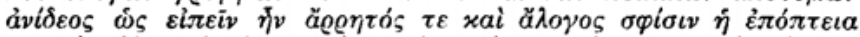

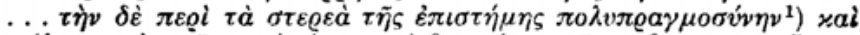

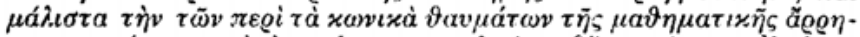

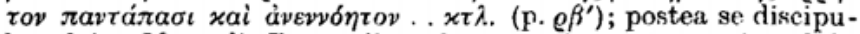
lum fuisse Manuelis Bryennii et ab eo maxime astronomiam didjcisse, mox autem, cum suo Marte Almagestum legere vellet, se intellexisse multa mathematices cognitione ad hoc studium opus

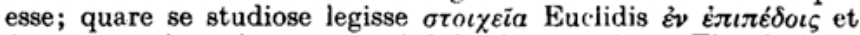

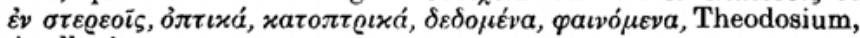
Apollonium.

Studiorum saeculi XIV testes sunt Isaac Argyrus et Barlaam (Studien p. 171 sq.), posterioris temporis Demetrius Cydonius et Theodorus Cabasilas, de quorum scholiis v. supra p. XXIV.

E studiis Byzantinorum etiam recensio brevior libri XII in cod. b servata (IV append. II p. $207 \mathrm{sq}$.) orta est, sed ante saec. VIII; nam certa sunt vestigia huius recensionis apud Arabes (v. Zeitschrift für Mathematik u. Physik XXIX, hist.-lit. Abt. p. 7 sq.). facta est ad codicem editionis antetheoninae (l. c. p. 13), sed tanto opere discrepat, ut ad scripturam antiquam eruendam parum utilis sit. quid de discrepantiis Arabum sentiam, in disputatione, quam citavi, pluribus dixi nec hic quaestionem difficilem retractabo, quippe quae editis demum versionibus Arabicis ad finem perduci possit. sed tamen fata Elementorum apud Arabes breviter narranda sunt (v. Klamroth, Zeitschrift d. deutschen morgenländ. Gesellschaft XXXV p. 270sq. et Steinschneider, Zeitschrift für Mathematik u. Physik XXXI, hist.-lit. Abt. p. 81 sq.).

Iam saec. VIII regnante Almansur chalifa Elementa Constantinopoli ad Arabes pervenerunt, si Hagio Khalfae III p. 91 fides est. saec. IX Hajjaj ben Jusuf ea Arabice interpretatus est, cuius interpretationis duae erant editiones; altera correctior iussu Mamuni chalifae, prior Haruni confecta erat; in altera sine dubio

1) Cf. scholium manu recentissima in $\mathbf{B}$ ad X 91 adscriptum (v. vol. V pars $2 \mathrm{nr}$. 405), ubi queritur aliquis de neglectis libris X-XIII.

\section{LXXIV}




\section{PROLEGOMENA CRITICA}

codicibus Graecis a Mamuno Cnopoli arcessitis usus erat Hajjaj (Hag. Khalfa I p. 81). Hajjaj non tam id studuit, ut verbum verbo redderet, quam ut liber discipulis aptus esset. saec. X Ishak ben Hunein denuo Elementa interpretatus est, Graeca verba pressius secutus, ceterum interpretatione Hajjajana tamquam fundamento usus. eius interpretationem Thabit ben Korra postea emendavit ope codicum Graecorum. novam interpretationum Arabicarum recensionem saec. XIII dedit Nassireddinus 'Tusi. libros XIV - XV Arabice interpretatus est Costa ben Lucca. quamquam praeter priorem editionem interpretationis Hajjajanae horum omnium codices non pauci exstant, tamen recensio Nassireddini sola edita est (Romae 1594 et, ut videtur, Cnopoli 1801); de duabus interpretationibus nonnulla dedit Klamroth, quae sufficiunt ad desiderium plenioris notitiae commovendum. Arabes enim codicibus recensionis antiquae usi sunt, qui in libris XI extremo et XII cum excerpto Bononiensi congruebant, sed addendo, demendo, mutando formam genuinam corruperunt, sicut demonstrasse mihi videor (Zeitschr. f. Math. u. Phys. XXIX, hist.-lit. Abt. p. 1-22). hae discrepantiae fontium Arabicorum a Klamrothio notatae sunt:

Omittunt analyses XIII 1-5, omnia lemmata, corollaria praeter VI 8; VIII 2; X 3, definitiones IV 3-7; VII 9 (vel 10); XI 5-7, $15,17,23,25-28$ (VI def. 5 et definitiones spurias libri V p. 1, 7; 3,17 habent, praeterea in libro VI novam definitionem sextam sine ullo dubio subditivam, sicut cetera Arabum additamenta, et in codd. Arabicis ut dubiam notatam), propositiones VIII 16, 17; X 7, 8, 13, 16, 24, 112, 113, 114, append. $5,27-28$; XI append. 3; XII 6, 13, 14 (cf. Bonon.), praeterea librum XV a p. 28, 16, omnes demonstrationes alteras, nisi quod in X $105-106$ (append. 25 - 26) loco genuinarum substitutae sunt, et in libro VII append. p. 235, $7-236,25$ (Nasireddin praeterea solus VI 12; X $27-28$ omisit). horum omnium nonnulla etiam in bonis codd. Graecis, maxime in $\mathbf{P}^{1}$ ), omittuntur vel tamquam dubia notantur ( $\mathrm{X}$ append. 5, XI append. 3, VII append. p. 235, 236), ex ceteris quaedam iam aliis de causis suspecta sunt (VII def. 10; X 112-114; append. $27-28$, demonstrationes alterae, lemmata corollariaque nonnulla, analyses XIII 1-5); sed quoniam maior pars eorum, quae ab Arabibus omittuntur, neque in Graecis fontibus antiquioribus vel saltim aetate supparibus abest neque omnino abesse potest, etiam in ceteris fides Arabibus detrahitur, praesertim cum interpolationibus deteriorum codicum Graecorum propriis non careant (VI def. 5 , VII def. analogiae et rationis ordinatae) et locos merito suspectos habeant (VI def. 2; X 115; XIII 6; cf. quod X append. 25-26 pro X 105-106 habent); fieri potest, ut haec omnia, quaedam iure, quaedam iniuria, suo Marte omiserint, cum constet, eos in verbis Euclidis servandis parum religiosos fuisse (v. praefatio cod. Bodl. arab. 280 saec. XIII fortasse a Nasireddino scripta apud Nicoll et Pusey, Catalog. codd. orientt. Bodl. II $^{2}$ p. 260 sq.). eadem de causa

1) Omittunt etiam additamentum Theonis VI 33 p. 233sq. 


\section{PROLEG OMENA CRITICA}

non magni momenti est, quod saepe ab ordine definitionum propositionumque Graecarum discrepant (in libro V deff. 11-12 falso permutant, sicut VI deff. $3-4$, in VII hic est ordo inde a def. 11: $12,14,13,15,16,19,20,17,18,21,22,23$, in XI hic: $1,2,3,4,8$, $10,9,13,14,16,12,21,22,18,19,20,11,24$; in propositionibus hae sunt discrepantiae: in libro III propp. $11-12$ coniunctae sunt $\left.^{1}\right)$, in V propp. 12-13 commutatae, in VI ordo est $1-8,13$, $11,12,9,10,14-17,19,20,18,21,22,24,26,23,25,27-30,32$, 31,33 , in VII: $1-20,22,21,23-28,31,32,29,30,33-39$, in IX: $1-13,20,14-19,21-25,27,26,28-36$ et ante 30 duse propp. novae, in X praeter omissas: $1-12,15,14,17-23,26-28$, $25,29-30,31$ in duas resoluta, 32 item, 33-111, 115; in XI: $1-30,31$ in duas resoluta, 32,34 in duas resoluta, $33,35-39$; in XII : $1-5,7,9,8,10,12,11,15,16-18$; cf. Bononiensis; in XIII: $1-3$ in binas resolutae, $5,4,6,7,12,9,10,8,11,13,15,14,16-$ 18) $)^{2}$ ).

Quantum fructus in singulis ad rem criticam ex interpretationi. bus Arabicis peti possit, non liquet, cum nondum editae sint; ex locis a Klamrothio citatis, qui cum Bononiensi comparari possunt, apparet, Arabes in vertendo Graeca aliquando ad verbum secutos esse.

De commentariis Arabum in Elementa omnia collegit Steinschneider l. c. p. 86 sq., nec opus est hic copias eius repetere; sed ex iis apparet, quantum Arabes Euclidi et tribuerint et debuerint; nam iis quoque magister erat mathematices.

Interpretationes Hebraicas duas idem commemorat l. c. p. 85, alteram incerti temporis e Latino factam, alteram saec. XIII ad interpretationem Ishaki confectam.

De interpretationibus Armenica et Persica nihil innotuit praeter brevem notitiam apud Wenrich, De auctor. Graecor. versionib. p. 184.

\section{Cap. IV}

\section{De Elementorum apud occidentales fatis}

Cum ceteris doctrinae Graecae operibus etiam Elementa ad notitiam Romanorum pervenerunt, quamquam iste populus inliteratus et vero amore scientise destitutus ,metiendi ratiocinan. dique utilitate huius artis modum terminavit", ut satis scite observavit Cicero (Tusc. I 5). primus apud Romanos Euclidem nominat Cicero (de orat. III 132), nec veri simile est, Elementa tum

1) Etiam in libro XIV saepius complures propositiones coniunguntur.

2) In communibus notionibus discrepare non videntur; postul. $4-5$ suo loco leguntur, xoıv $\check{z} w$. 9 non inter postulata legitur, sed eodem loco, quo in editione mea. 
Latine conversa fuisse vel omnino in studiis Romanorum locum magnum obtinuisse; nam etiam quod Quintilianus I 10, 34 sq. de geometriae utilitate dicit, ultra primas mathematices notiones non progreditur (velut cum sic incipit, ,in geometria partem fatentur esse utilem teneris aetatibus. agitari namque animos et acui ingenia et celeritatem percipiendi venire inde concedunt; sed prodesse eam non ut ceteras artes, cum perceptae sint, sed cum discatur, existimant"). et quam pauca geometriae excerpta agrimensoribus Romanis satisfecerint, apparet e Balbi libro de mensuris (Agrimens. I p. 97 sq.), ubi definitiones nonnullae ex Elem. lib. I afferentur. ceterum M. Cantor in libro egregio „Die römischen Agrimensoren und ihre Stellung in der Geschichte der FeldmeBkunst"“, Leipzig 1875 demonstravit, eos pleraque non ex Euclide, sed ex Herone petisse ingenio operisque genere iis magis familiari. etiam quae in fragmento Censorino adscripto (Hultsch, Censorinus, Leipzig 1867, 60-63) ex Elementis transsumpta sunt, intra definitiones, postulata, communes conceptiones se continent. tamen Elementa sensim etiam apud Romanos in circulum artium liberalium recepta sunt (cf. Ussing, Erziehung u. Jugendunterricht bei den Griechen u. Röm. p. 133); nam Martianus Capella VI 724 haud obscure innuit, Elementa tum omnibus philosophis certe familiaria fuisse. sed sine dubio Graece legebantur. nam quae Capella VI 708sq. habet, ipse e Graeco fonte excerpsit, ut Graeca verba plurima ostendunt (conferri potest, quod I def. 1 falso vertit: ,punctum vero est, cuius pars nihil est"); sed dubito, an non ex Euclide ipso (cf. Studien p. 202 sq.). in quibusdam cum Herone aliisque fontibus vetustis consentit, velut quod quinque tantum postulata, tria axiomata habet; I p. 2, 2 cum Ammonio, Philopono, aliis, p. 4, 16 cum Proclo aliisque, p. 5, 6 cum F et Proclo consentit; in I def. 15 primum tantum glossema habet,

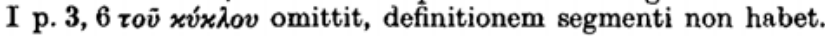

Ceterum eodem fere tempore conatum esse virum aliquem doctum Elementa Latine convertere constat e codice palimpsesto Veronensi nr. 40 (Blume, Iter ital. I p. 263), quem descripsit Guilelmus Studemund. continet fragmenta versionis liberrimae vel potius redactionis novae Elementorum libb. XI-XIII (in codice numerantur XIV et XV, quod quo modo factum sit, non intellego, nisi forte pars est maioris operis encyclopaedici) alio propositionum ordine. codex palimpsestus saeculi fere IV esse fertur et sine dubio ipsum exemplar interpretatoris est (haec Cantor, Vorlesungen üb. Gesch. der Math. I p. 478 sq.; nam Studemundus ipse nondum haec fragmenta edidit). num propositum suum ad finem perduxerit, nescimus, neque omnino de ratione, quae inter hanc interpretationem ceterosque fontes intercedit, quidquam constat. primus, ut videtur, Latinam interpretationem Elementorum dedit Boetius (v. Cassiodorii Var. I 45 translationibus enim tuis ... Nicomachus arithmeticus, geometricus Euclides audiuntur Ausoniis; cf. idem de geometr. p. 577 Euclidem translatum in Romanam linguam idem vir magnificus Boetius dedit); quadrivium 
enim, quod vocatur, e fontibus Graecis 4 operibus Latine edidit. sed quae in codd. plurimis nune fertur geometria Boetii, ne talis quidem, qualis a Friedleinio e cod. Erlangensi $288 \mathrm{~s}$. XI edita est (de codd. interpolatis v. Röm. Feldmesser I p. 377 sq., II p. 64 sq.; p. $79 \mathrm{sq}$.), a Boetio profecta est, sed saec. XI ex fontibus compluribus conflata (v. Weissenborn, Abhandl. z. Gesch. d. Math. II p. $185 \mathrm{sq}$.; cf. quae dixi Philolog. XLIII 507sq.). sed quamquam hoc opus et recens est et mendosissime in codd. scriptum, tamen vestigia sunt scripturae bonae et antiquae (glossemata in I def. 15 p. 2, 10 et 12 om., item $\tau o \bar{v} x \dot{v} x \lambda o v$ et def. segmenti p. 3, 6; I p. $3,9,12 ; 4,16 ; 5,6$ al. cum Proclo consentit; quinque postulata et tria axiomata sola habet) ${ }^{1}$ ). quam unde habeat, nescimus. sed hoc constat, falsarium interpretationem Latinam Elementorum habuisse, unde $11-3$ petiverit (p. $390-393$ ed. Friedlein). et interpretationem Jatinam saec. X - XI exstitisse, ostendit. M. Curtze (Bursians Jahresberichte, 1884, p. 19; cf. quae de cod. Monac. 560 dixit Friedlein, Boet. p. 373). ${ }^{2}$ ) has interpretatione Curtzius eos usos esse contendit, qui interpretationes medio aevo usurpatas confecerunt, Adelhardus et Campanus. hos viros, si summam spectes, e fonte Arabico Elementa interpretatos esse, vel ex vocabulis Arabicis apud eos obviis pro certo concludi potest (Studien p. 178) ${ }^{3}$ ), sed quae ratio inter eos intercedat, nondum exploratum est; neque enim sententia Weissenbornii (Abhandl. z. Gesch. d. Math. II p. 141 sq.) probari potest, utrumque suo Marte Elementa ex Arabico transtulisse. immo ita se res habere videtur, ut in codd. quibusdam ${ }^{4}$ ) significatur, Adelhardum (saec. XII) interpretem esse, Campanum Novariensem (saec. XIII) commen. tatorem. ${ }^{5}$ ) sed de hac re nondum satis explorata hic pluribus disputare nolo. Campanus igitur sive Adelhardus multis locis cum interpretationibus Arabicis consentit, sed teste Klamrothio nullam earum, quas hodie novimus, fideliter exprimit. cum praeterea co-

1) Boetius ipse axiom. 6 et 9 novit (Arithm. p. 91, op. p. 165), I def. 14 (op. p. 181), def. 2 (op. p. 145), def. 3 (op. p. 146), def. 5 (op. p. 146), def. 15 (sine glossematis) et def. 22 (op. p. 187) citat.

2) Geometriam Boetii continet etiam cod. Harleian. 3595 fol. $57 \mathrm{sq}$.

3) Ubi scriptum oportuit: helmuayn $\varrho o ́ \mu \beta o \varsigma$, similis helmuayn

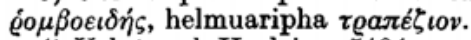

4) Velut cod. Harleian. 5404: per Adelhardum Batonensem ex arabico in lat. translat., Harl. 5266 m. rec.: liber Euclidis $\overline{\text { phy }}$ quem transtulit adelardus batoniensis de arab. in lat., Mus. Brit. add. 22783: geometria Euclidis cum commento Campani explicit, cod. Bodl. Canon. Lat. 309: explicit geometria euclidis cum commento Campani novariensis.

5) Interpretationem Gerardi Cremonensis (saec. XII; liber Euclidis tractatus XV, v. Boncompagni vita ed op. di Gherardo Cremon. p. 5) non habemus. etiam Leonardus Pisanus ab Arabibus pendet (Weissenborn, Philol. Anz. XV 44).

\section{LXXVIII}




\section{PROLEGOMENA CRITICA}

dices non leviter ab editione (Venetiis 1482, repetita Vincentiae 1491) dissentiant necdum collati sint, non tanti esse putavi editionem illam cum codd. Graecis conferre. hoc tantum commemorabo, Campanum multum et in ordine propositionum et in demonstrationibus a codd. nostris differre et plurima additamenta habere. ${ }^{1}$ ) sed restant vestigia fontis purioris, velut quod additamentum Theonis in VI 33 non habet; alia quaedam suis locis notavi, sed, ut nunc est, in rebus criticis parum utilis est.

Ex hac igitur interpretatione medium aevum Euclidis funda. mentorumque mathematices notitiam pro sua facultate petebat; quare et codices plurimi eius exstant (velut in bibliotheca Amploniana Erfurtensi, quae eos continet libros, quibus litterarum studiosi saec. XV in studiis utebantur, duo exstant), et prima om. nium librorum mathematicorum prelo impressa est. primus apud occidentales Elementa Graece exstare novit fohannes Boccatius (Comm. sopra la commedia di Dante I 404), sine dubio a Barlaamo magistro suo, qui de Euclide scripsit, edoctus.

Deinde Iohannes Regiomontanus, cuius exemplar interpretationis Adelhardi nunc in bibliotheca civitatis Norimbergensis asservatur, et qui eam edere voluit emendatam (Gassendi op. V p. 530 Euclidis elementa editione Campani evulsis tamen ple. risque mendis, quae proprio etiam indicabuntur commentariolo ${ }^{2}$ ), in Italia, sine dubio apud Bessarionem amicum, Graecos codices vidit et animadvertit, quantum a Latinis Campani discreparent (v. epistola eius ad Chr. Roderum Hamburgensem a. 1471 scripta apud Murr, Memorabilia bibliothecar. Norimberg. I p. 190sq., ubi Campanum graviter vituperat; velut p. 191 „quem scrupulum“ inquit „et Campanus animadvertens hoc principium - agitur de aǐr. 5 - inter petitiones stolide collocavit, quamvis Greci inter communes sententias ordinarint. sed Arabes nonnulli a ministerio demonstrationis penitus reiecerunt hoc proloquium aliter quidem equidistantes lineas definiendo").

E codice Graeco primus Elementorum partes Latine versas edidit Georgius Valla, qui libros XIV - XV aliis interpretationibus suis adiunxit Venetiis 1498 (Neue Jahrb., Suppl. XII 377) et deinde in opus ingens de expetendis et fugiendis rebus (Venet. 1501) non paucas propositiones cum demonstrationibus scholiisque recepit (v. ib. 379 sq.). usus est cod. Mutin. III B 4 saec. XV (v. Cenni storici della bibl. Estense p. $11 \mathrm{nr}$. LVI: codice cartaceo in $4^{\circ}$ del secolo XV. Fu di Giorgio Valla, poscia di Alberto Pio; de fatis

1) Cf. J. E. Murdoch, The medieval Euclid. Salient aspects of the translations of the Elements by Adelard of Bath and Campanus of Novara, Revue de Synthèse 89, Paris 1968, 67-94.

2) Editio rarissima Venet. 1509 Campanum continet a Luca Paciolo emendatum, v. Weissenborn, Die Ubersetz. d. Eukl. durch Campano u. Zamberto p. 30 sq.; ib. p. 56sq. describitur editio Parisina a. 1516, quae Campanum et Zambertum coniungit (repetita cum mutationibus paucis Basil. 1537, iterum ib. 1546). 


\section{PROLEGOMENA CRITICA}

bibliothecae Vallae v. Philolog. XLII 432sq.). nam glossemata quaedam huius codicis propria habet Valla, velut I p. 15, $13 \tau \dot{\jmath}$

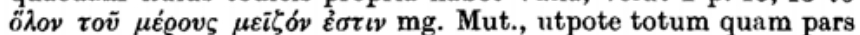

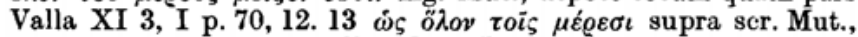
utpote totum partibus Valla XI 12, I p. 69, 17. $18 \omega \dot{\omega} \tau \varepsilon \tau \rho \alpha \gamma \dot{y} v o v$ $\pi \lambda \varepsilon v \varrho \alpha ́$ supra scr. Mut., utpote quadrati latus Valla XI 12; ef. praeterea I p. 70, $10 \Gamma \Delta E B] \gamma \delta \beta \varepsilon$ Mut., cdbe Valla; $11 \delta \iota \eta ́ \gamma \vartheta \omega]$

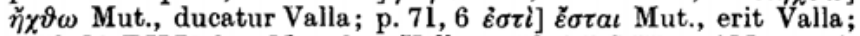
p. 71, $18 \Gamma H B] \beta \eta \gamma$ Mut., bg c Valla; p. 72, $6 B \Gamma H] \eta \gamma \beta$ Mut., g cb Valla; p. 72, 13.14 xai-IB om. Mut., Valla; p. 72, 14 . 15 rà ă̊a$\Gamma B$ om. Mut., Valla. de scholiis eius alibi videbimus.

Cum menda editionis Campani codicibus Graecis magis magisque cognitis semper plura deprehenderentur et appareret, eum Euclidem neque genuinum neque totum dedisse, desiderium veri Euclidis cognoscendi ortum est, quo motus Bartholomaeus Zambertus Venetus Euclidem totum e codicibus Graecis sibi interpretandum sumpsit (Venet. 1505), quem librum rarissimum diligenter descripsit Weissenborn (Die Ubersetzungen des Euklid durch Campano und Zamberto p. 12sq.), qui idem (1. c. p. 21 sq.) eos locos collegit, ubi Zambertus in adnotationibus suis Campanum eiusque interpretandi morem vituperat. ${ }^{1}$ )

Titulus est (v. Weissenborn p. 13) „Euclidis megarensis philosophi Platonici mathematicarum ianitoris. habent in hoc volumine quicunque ad mathematicam substantiam aspirant, elementorum libros XIII cum expositione Theonis insignis mathematici, quibus multa, quae deerant, ex lectione graeca sumpta addita sunt, nec non plurima perversa et prepostere voluta in Campani interpolatione ordinata digesta et castigata sunt " cet. praeterea affero e praefatione catoptricorum (Weissenborn p. 24, ed. a. $1546^{2}$ ) p. 504): „sicut lectio sese habet graeca, sic veritatem colentes nuda, pura, sincera et fideli sumus interpretatione interpretati. noluimus enim eos imitari, qui ex auctoribus aliqua decerpunt, aliqua omittunt et aliqua permutant" cet. et e praefatione Datorum (Weissenborn p. 25, ed. a. 1546 p. 542): „Euclides namque Megarensis Mathematicus praeclarissimus, qui omnium mathematicarum disciplinarum unus est qui nobis fores reserat, in primis nimis perverse interpretatus studentium animos pluribus annis ambiguos tenuit. nam cum illud, quod illius esse asseritur vo. lumen studentes legerent, miris larvis, somniis et phantasmatibus, quibus ille interpres barbarissimus illud refersit, offensi neque auctori fidem adhibebant neque illi detrahere audebant. quare cum nos his disciplinis operam per plures annos accommodaverimus volentesque nostris laboribus studentium communi utilitati con-

1) Utrum idem ex his locis recte concluserit, Zambertum nescivisse, Campanum ex Arabico Euclidem transtulisse, necne, nunc quidem dubito.

2) De editionibus Campanum et Zambertum coniungentibus v. supra p. LXXIX not. 2.

\section{LXXX}




\section{PROLEGOMENA CRITICA}

sulere, ipsius Euclidis elementorum volumina tresdecim ex Theonis traditione non minoribus vigiliis quam laboribus, quibus per septennium insudavimus, ex graecia in Italiam deduximus." quibus verbis cum Campanus haud obscure significetur, apparet causa, cur Zambertus suam interpretationem confecerit. quo co. dice Graeco in Elementis (nam in prolegomenis Marini ad Data codicem „e bibliotheca senatoria", h. e. Marciana Venetiis, habuit, ut ipse dicit p. 537 ed. Basil.) usus sit, neque ipse dicit, neque ego adhue indagare potui. hoc tantum affirmare possum, eum codicibus Marcianis usum non esse; neque enim inter eos est, qui plus quam XIII libros contineat, cum tamen Zambertus etiam libros XIV - XV interpretatus sit, nec in locis memorabilibus, ubi seripturam Marcianorum enotavi, cum ullo prorsus et constanter consentit. ne eo quidem codice, qui nune Lugduni Batavorum as. servatur (Leid. 7), usus est, quamquam ipso eum descripsit; nam obstant temporum rationes, quoniam interpretationem absolverat a. 1500 (v. Weissenborn p. 16) et codicem descripsit a. 1504

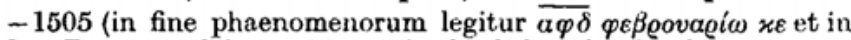

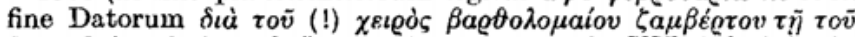

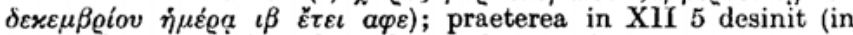
$\mu \varepsilon i \zeta$ ov IV p. $\left.93, \dot{5})^{1}\right)$. sed quidquid id est, codex eius deterioribus adnumerandus est, quippe qui plerasque interpolationes Theoninorum habeat, velut II append. p. 233, definitionem rationis ordinatae; cf. I p. 29, 3. memorabile videtur, quod in XI 38 p. 73, 13 et $x v ́ \beta \rho v$ et $\pi \alpha \varrho \alpha \lambda \lambda \eta \lambda \varepsilon \pi \iota \pi \varepsilon \delta \delta o v$ habuit; scribit enim p. 386: , si solidi parallelepipedi“ cet., deinde: „aliter. si cubi“ cet. (in propositione illud tantum habet).

Graeci Euclidis editio princeps prodiit Basileae 1533 apud Hervagium cura Simonis Grynaei, qui in praefatione dicit, se duobus codicibus usum esse, quorum ,alterum Lazarus Bayfius ${ }^{2}$ ) Venetiis, alterum Parrhysiis Ioann. Rvellius ${ }^{3}$ ) amicis, mihi ipsi Procli commentaria Oxonii Ioann. Claymundus candide suppeditabat, viri optimi et humanissimi" cet. eos mihi contigit reperire. codicem enim Venetum fuisse Marc. 301 discimus ex errore ed. Basileensis quodam II p. 8, 12, ubi pro $\tau \dot{\alpha} K, \Lambda$ habet $\tau o \bar{v} x \lambda$; et hoc in solo Marc. 301 ita scriptum inveni. hine simul apparet, ipsum codicem hunc fundamentum esse editionis, et ,exemplar alterum", unde in mg. scripturas discrepantes excerpsit Grynaeus, Parisiis

1) Continet fol. 1-173 Elem. I-IX, fol. 173-174 scholia quaedam in lib. X; fol. 175-177 vacant; fol. 178-315 Elem. X-XII 5; fol. 316-318 vacant; fol. 319-331 catoptrica, fol. $331-360$ phaenomena; fol. $361-383$ opticorum editionem novam cum scholiis, fol. 383 - 433 Data; fol. $434-438$ vacant; fol. 439 452 excerpta e Proclo; fol. 453 vacat; fol. $454-459$ Marinum in Data. fol. 439r legitur ,a me Io. Francisco Asulano“.

2) Lazare de Baîf, vir doctus, qui tum legatus regis Galliae apud Venetos fuit; $\dagger 1547$.

3) Jean Ruel medicus Gallus 1479-1537, Graece doctus. 


\section{PROLEGOMENA CRITICA}

quacrendum esse. est cod. Paris. Gr. 2343 chartac., Memmianus s. XVI, qui continet Elem. I-XV cum scholiis nonnullis, quae in textum recepta sunt; in primo folio rubro colore scriptum est

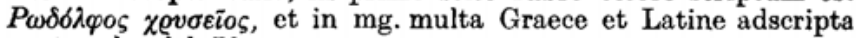
sunt, velut fol. 70

perfer ulixeo reflantem pectore sortem in tumido quisquis nasque renasque salo

Aenosinus Nauarchus.

Hic codex igitur semper cum scripturis is concordat, quas Gry. naeus in mg. ex ,exemplari altero" enotavit, velut

I p. 14, 14. 15 Venet. 301 et Grynaeus cum mea editione con-

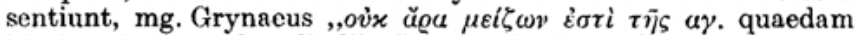

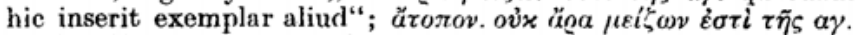

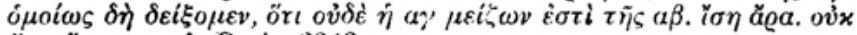
ă $\varrho$ a ăvı

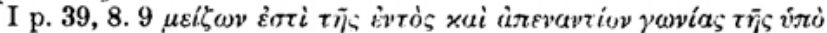

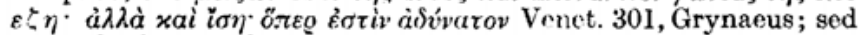

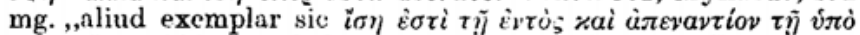

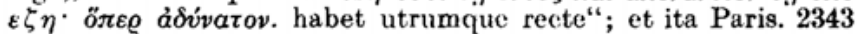
(corr. m. 2).

I p. 79, 13 xai $\tau \dot{a} K \Delta a ̆ \varrho \alpha x \tau \dot{\lambda}$.] Venet. 301, Grynaeus; mg. ,,va-

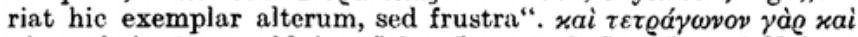

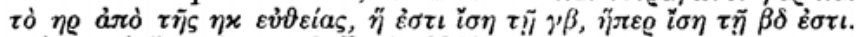
xai $\tau \tilde{\omega} \times \delta$ ăoa $\tau \dot{\alpha} \eta \varrho x \tau \lambda$. Paris. 2343.

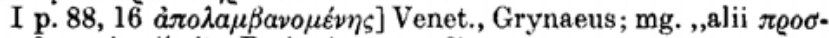

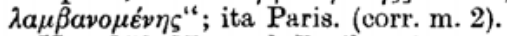

II p. 158, 17 sq. ed. Basil. scripturam codd. $\mathrm{BV} \varphi$ habet, sed in mg. "non habet exemplar alterum et videtur nibil huc pertinere"; Paris. cum nostris concordat.

III append. 5 p. 216 hab. ed. Basil. ut $\iota \gamma$, sed mg.,,in altero exemplari lemma est. itaque variat numerus deinceps" ; sine numero Paris.

III append. 8 p. 218 hab. ed. Basil., mg. ,non habet exemplar alterum"; om. Paris.

III append. 9 p. 218 hab. ut $x \eta$ ed. Basil., nug. ,hanc exemplar alterum non habebat nec videtur esse autoris"; om. Paris.

III append. 10 p. 219 hab. ed. Basil.; mg. ,non habet alterum exemplar"; om. Paris.; item in III append. 11 et 12 p. 220; 14 p. $222 ; 15$ p. 223.

III append. 18 p. $224 \mathrm{mg}$. Grynaeus ,,addit exemplar alterum"; om. Venet., hab. Paris.; item III append. 19 p. 225; 20, 21, 22 p. 226.

IV p. 19, 2 ed. Basil. scripturam codicis b habet, sed in mg. nostram (,alterum graecum exemplar sic"), quae etiam in Paris. est.

$\mathrm{E}$ cod. Parisino 2343 praeterea petivit, quod ad XI 1 in $\mathrm{mg}$.

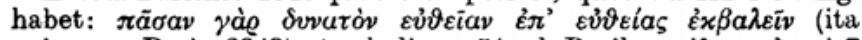

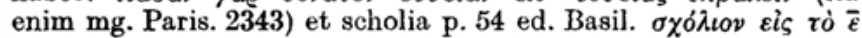
à $\delta \eta^{\prime} \lambda o v$ (Paris. fol. 56 sine titulo, mg. m. 2 , in librum quintum 


\section{PROLEGOMENA CRITICA}

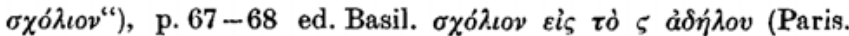
fol. 70 cum hoc ipso titulo multisque correctionibus manus 2, quae in ed. Basil. receptae sunt ${ }^{1}$ ).

Sed interdum scripturam codicis Parisini recepit Grynaeus et per ,exemplar alterum" codicem Venetum significavit. velut III append. $25-26$ p. $229 \mathrm{sq}$. (,non habet alterum graecum exemplar" ,alterum graecum exemplar non habet" mg. Grynaeus; in Venet.

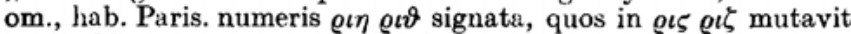
m. 2 addito in mg. ,,om. hoc ${ }^{2}$ ) alterum graecum exemplar").

Praeter Venet. 301 et Paris. 2343 Grynaeus etiam Zambertum consuluit. v. ad IX 19 ,quia Zampertus Graecum sine dubio exemplar secutus exacta divisione membrorum hic utitur et singula membra demonstrationibus exequitur, voluimus eam lectionem inserere: est enim pernecessaria; licet neutrum nostrum exemplar tale quicquam haberet" (hic Zambertus sine dubio errorem codicum perspexit et de suo meliora restituit). item ad IX $30 \mathrm{mg}$. ,addit hic quaedan Zampertus, quae non videntur Euclidis" (in fine propositionis Zambertus quaedam addidit), ad X 32 ed. Basil. (III p. 51, 7) ,sic habet exemplar latinum" (et Zamb. et ed. Basil. cum meis codd. concordant; de Venet. et Paris. nihil compertum habeo), ad X $\varrho$ เ aliam rursus eiusdem demonstrationem latinum exemplar" (de suo addidit Zambertus „priorum dilucidiorem explanationem“" per numeros, p. 344 ed. 1546, ubi mg.,,Graecus non habet"), ad XI 26 (IV p. 44, 8.9) ,,sic habet latinum $\vartheta x \lambda \tau \bar{\eta} \dot{v} \pi \dot{\delta} \zeta \eta \gamma$, sed eodem tendunt" (,qui sub $\theta x \lambda$ ei qui sub $\zeta \eta \gamma$ est aequalis"Zambertus p. 370), ad XI 30 (IV p. 49, 24),,variat exemplar latinum, sed eodem recidunt" (Zambertus p. 373 in litteris differt), ad XI 31 (IV p. 51,9) ,ponit enim exemplum in non prorsus similibus solidis, quod interpres latinus hic omisit" (additamentum codicis B habet et Grynaeus et Zambertus), ib. ,stantes hic la(ti)num addidit, verum nihil est" (,,stantes" apud Zambertum p. 374 respondet

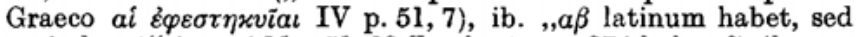
perinde est" (pro $A M$ p. 51, 19 Zambertus p. 374 hab. $\alpha \beta$ ), ib. „, $\varrho \varphi$ latinum habet, sed est idem" (sed in ed. a. 1546 est $\psi v$ ut in ed. Basil.), ad XI 40 (IV p. 76, 11) ,latinum $\gamma \vartheta$, sed perinde est" (sed Zambertus p. 387 no habet, sicut Grynaeus; his duobus locis igitur interpretatio Zamberti in editione Hervagiana a. 1546 ad Graecam editionem a. 1533 correcta est).

Cum et Venet. 301 et Paris. 2343 deterrimus sit, apparet, quam nulla sit auctoritas editionis principis. sed tamen diu fons fundamentumque textus Graeci Elementorum mansit. nam quae deinde

1) Itaque sine dubio ipse Paris. 2343 in manibus typothetarum fuit ad cod. Venetum supplendum. in Paris. fol. $93^{\mathrm{v}}$ scholium est

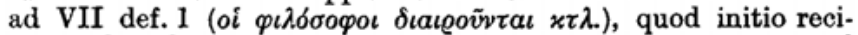
pere voluit Grynaeus; nam in principio m. 2 correctum est; sed $\mathrm{ab}$ instituto destitit.

2) Haec duo verba pro certo dignosci non possunt. 
prodierunt editiones, non eo consilio factae sunt, ut verba Euclidis e codicibus Graecis integriora restituerentur, sed ut mathematices studiosis modico pretio habile compendium pararetur Elementorum, quae tum in scholis a professoribus mathematices vulgo docebantur. eius generis hae sunt:

Euclidis elementorum libri XV. Romae 1545; ,Antonio Altovito in primis eruditissimo Angelus Caianus s. p. d." (ex praef. affero: omnes enim tibi affert elementorum libros - hos XV esse non ignoras - cum integros tum emendatos tum etiam a sexcentis rebus quasi purgatos, quae nec Euclidis ingenium illud prope divinum neque perspicuitatem ... redolere penitus videbantur); continet propositiones solas (sine demonstrationibus) omnium XV librorum Graece.

Euclidis elementorum geometricorum libri VI conversi in latinum sermonem a Ioach. Camerario, edebat Lipsiae Georg. Ioach. Rhet(icus) 1549; in praef. ,nobis hoc potissimum in adornanda interpretatione nova consilium fuit, ut studiosi harum diaciplinarum ad Graecam linguam discendam invitarentur".

Euclidis Elementorum libri XV Graece et Latine. Lutetiae 1558 (repetita ib. 1573, 1598); in praef. (ad candidum lectorem St. Gracilis praefatio, Lutet. 4 Id. Apr. 1557) Gracilis narrat, opus susceptum esse hortante Io. Magnieno professore mathematices Parisiensi; in libb. I-VI se temporis angustiis coactum nihil fere mutasse, in ceteris autem emendasse, ,quae subobscure vel parum commode in sermonem latinum e graeco translata videbantur", in lib. X vero interpretationem Petri Montaurei (bibliothecarii Aurelianensis $\dagger 1571$, de cuius studiis Euclidianis v. Heilbronner, Hist. math. p. 159) totam recepisse. propositiones solas habet, additis scholiis quae vocat nonnullis, h. e. III p. 126, 1sq.; p. 200, 11sq.; IV p. 185,5 sq.; V p. $17,11-19,4$; p. $28,16-29,16$.

Euclidis quindecim Elementorum Geometriae primum ex Theonis commentariis Graece et Latine. cui accesserunt Scholia ... authore Cunrado Dasypodio Scholae Argentinensis professore. Argentorati 1564 (repetita cum Herone ib. 1570 sive, ut in aliis exemplaribus est, 1571). ex praef. ed. prioris ,annis viginti sex nostri Gymnasii consuetudo fuit, ut, qui ex classibus ad publicas lectiones promoventur, prinum audiant Euclidis librum ... . fui et ego quoque nostro Typographo author et suasor, ut, cum nulla amplius extarent exemplaria, hunc libellum imprimeret, ne bona et fructuosa scholae rostrae constitutio intercideret". ex. praef. alterius ,sed et hunc primum Elementorum Euclidis librum in lucem nunc edo, cum propter ea, quae ante sunt dicta, tum etiam quod hic potissimum liber in omnibus fere Gymnasiis praelegatur, in nostris vero scholis iis, qui in prima sunt curia, proponatur".

Euclidis qvindecim elementorum Geometriae secundum ex Theonis commentariis Graece et Latine ... per Cunr. Dasypodium ... Argentorat. 1564 (cum Barlaamo). ex praef. ,mihique satis erit ... in studiosorum gratiam aliquid fecisse".

Propositiones reliquorum librorum Geometriae Euclidis Graece

\section{LXXXIV}




\section{PROLEGOMENA CRITICA}

et Latine in usum eorum, qui volumine Euclidis carent. Per Cunr. Dasypodium. Argentorat. 1564. ex praef., ,quare ut in duobus prioribus libellis, quos in lucem edidi, bonos adolescentes adhor. tatus sum ad studium Geometriae, ita et hoc in loco faciam .... verum ne hortator solum, sed et adiutor essem, volui in gratiam studiosorum propositiones reliquorum Euclidis librorum Graece et Latine edere, eo sane consilio, quod cogitarem, mutilatum quippiam esse, si primus et secundus liber tantum imprimeretur ..... necesse est, ut eadem frequenti lectione sibi quisque faciat familiaria; molestum vero est integrum Euolidis volumen perpetuo hinc et inde circumferre; arbitrabar igitur, si in libellum redigeretur minorem, commodius esse omnibus geometriae studiosis haec percipere elementa."

Has omnes editiones e Basileensi pendere, apparet ex erroribus quibusdam, qui, ut ostendit collatio codicis Veneti 301, in ed. principe commissi sunt a typographis, et qui in illis editionibus servati sunt, velut II p. 3, 19 yivpral] yiverat ed. Basil., Caian., Rhet.,

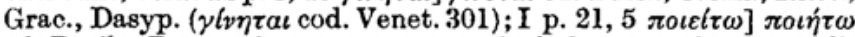
ed. Basil., Dasyp. itaque quae propria habent, coniecturae edi-

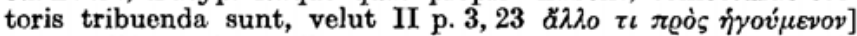

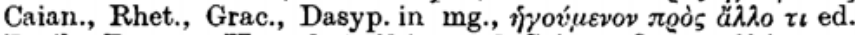

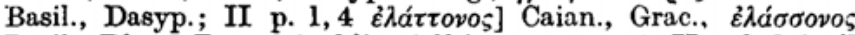

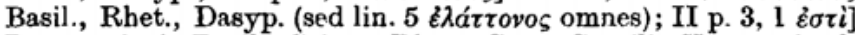

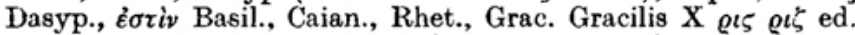
Basil. omisit, sine dubio nota illa marginis Basil. permotus (sic etiam Dasyp. $)^{\mathbf{1}}$ ). quod idem quarto loco inter deff. libri V habet

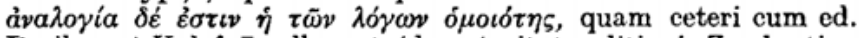
Basil. post $V$ def. 7 collocant, id auctoritate editionis Zamberti a. 1546 fecit. hoc quoque commemorandum est, I p. 29, 9 छ $\pi i$ in ed. Basil. compendio impressum esse; quo factum est, ut Caian. et Rhet. ảं ò ederent (ย́ri Grac., Dasyp.). in libb. XIV - XV ed. Basil. solum 4 et 5 propositiones numeris signavit; quare ne Caian., Grac. quidem plures habent (Dasyp. XIII tantum libros habet).

Dasypodius tamen interdum editionem Basil. ex Proclo emen-

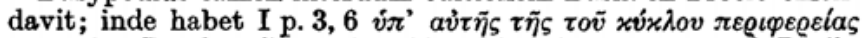

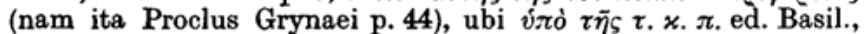

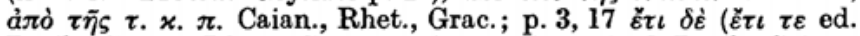
Basil., Caian., Rhet., Grac.), p. 22, 8 żлi ( $\pi \varepsilon \varrho i$ ed. Basil., Caian.,

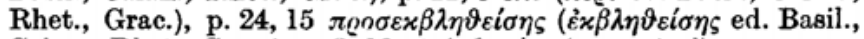

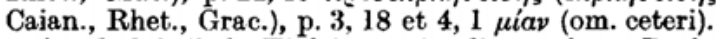

Apud Scheibel, Einleitung in die mathem. Bücherkenntniss I p. 6sq. aliae quoque editiones textus Graeci commemorantur. sed ea saltim, quae a. 1530 Basileae a Grynaeo edita esse dicitur (auctore Heilbronnero p. 159), numquam exstitit. editiones a. 1536 Orontii Finaei, a. 1550 Scheubelii (libb. I-VI), a. 1554 Parisiis (elem. arithmet.), a. 1573 Dasypodii numquam vidi.

$\mathrm{Ne}$ ex interpretationibus quidem ullum subsidium critices peti

1) Caianus om. $\mathrm{X} \iota \delta$ ed. Basil. nota ad $\mathrm{X} \iota \gamma$ deceptus. 


\section{PROLEGOMENA CRITICA}

potest. editio Basil. a. 1546 Campani et Zamberti sane a Christiano Herlino mathematico correcta est; sed praeterquam quod interdum Zambertum ad edit. Basil. a. 1533 correxisse videtur (v. supra p. LXXXIII), nihil fecit, nisi ut hic illic, ubi Zambertus vocabulum Graecum minus commode interpretatus erat, hoc ipsum ex ed. Basil. a. 1533 apponeret, velut p. 113 ad V def. 4 Zamb. ,proportio vero est rationum identitas" addidit ó $\mu$ otót $\eta \varsigma$, quod ex V def. 8

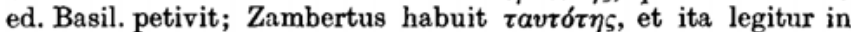
omnibus codd., qui hanc definitionem quarto loco habent. ${ }^{1}$ ) de Herlino v. praef. Hervagii: ,,collatum est itaque exemplar Iacobi Fabri Stapulensis ductu Parisiis ante aliquot annos excusum [v. supra p. LXXIX not. 2] ad fidem Graeci exemplaris [h. e. ed. Basil. 1533] a doctissimo viro Christiano Herlino ${ }^{2}$ ) mathematicarum disciplinarum publico apud Argentinenses professore, cui acceptum feras, quicquid hic aut ad Graecum exemplar aut alioqui docte restitutum videris."

Iam vero Nicolaus Tartalea, qui a. 1565 italicam Elementorum interpretationem edidit (repetita Venet. 1585), ne editionem quidem Graecam adiit; certe eam non nominat, ubi exemplaria, quae auditores in manibus habeant, enumerat (seconda lettione 11 : „la prima tradottione dal Campano", ,,la seconda fatta da Bartolomeo Zamberto Veneto, che vive ancora", „la stampa di Parise over d'Alemagna, nella quale hanno incluso le predette ambedue traduttioni, ma per un certo modo, qual è piu presto atto a generare confusione in cadauno studente che altramente", ,la nostra traduttione fatta in volgare" nam editione altera 'Tartaleae utor).

Inter interpretes solus Federicus Commandinus codice Graceo usus est (Euclidis Elementorum libri XV una cum scholiis antiquis. Pisauri 1572, ed. altera ib. 1619; e praef. ed. pr. ad Franciscum Mariam II Urbinatum principem: ,,Orontius quidem Phinaeus ... priores tantum sex libros nulla graeci codicis ratione habita [itaque de editione graeca a. 1536 iusta est causa dubitandi, v. supra p. LXXXV] edidit. Iacobi vero Peletarii in eadem re labor eo etiam minus probatur, quod Campani editionem ex arabica conversam lingua magis quam graecam sequi voluerit. alii autem peracuti sane ingenii homines ảvatvorıı geometricas in priores sex libros conscripserunt, cetera tamen non sunt prosecuti. at Candalla ... parum tamen, ut audio, eo nomine commendatur, quod longius iter ab Euclide averterit et demonstrationes, quae in graecis codicibus habentur, velut inelegantes et mancas suis appositis reiecerit" ") $\left.{ }^{3}\right)$.

1) Hinc simul apparet, quo iure supra p. LXXXV contenderim, Gracilem h. l. hanc editionem secutum esse.

2) Idem vir cum Dasypodio edidit: Analyses geometriae sex librorum Euclidis. Argent. 1566, in quo libro propositiones libb. I - VI Graece continentur; textus idem est atque in ceteris Dasy. podii editionibus.

3) Candallae interpretatio prodiit Paris. 1566 (repetita ib. 1578).

\section{LXXXVI}


Commandinus igitur, qui omnino de mathematicis Graecis optime meritus est, praeter ed. Basil. a. 1533 (quam citat fol. 68 , ,in

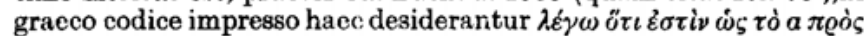

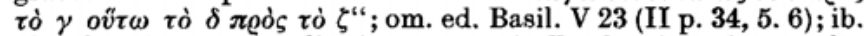
,hoc loco in graeco codice impresso et in Zamberti versione multa inseruntur supervacanea, quae a nobis consulto omissa sunt"; II p. 34, 16- 35, 8 enim, ubi additamentum p. 34 in app. erit. ad 17 not. habet, in formam breviorem redegit Comm., sine dubio suo Marte; fol. 247 b „Graecus codex corruptus est, qui haec habet“; sequitur scriptura ed. Basil. p. 261, 40-43 (V p. 16,12-14), quam corrigit) etiam codicem Graecum habuit, sed tam raro eum com-

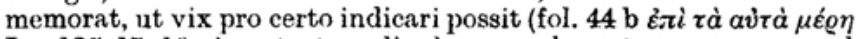
I p. $125,17.18$, in vetusto codice haee non leguntur, quamquam ad demonstrationem necessaria sint"; hab. Basil., Zamb., m. 2 V; fol. $131 \mathrm{~b}$,quamquam hoc ex illo perspicue appareat, tamen secundum lemma, quod in Graecis codd. invenitur, hoc loco apponere non inutile iudicavimus"; est III append. 10, quod ad X $x \zeta$ habet alter cod. Grynaei, e nostris $\mathbf{V}$ solus); de scholiis eius alibi videbimus.

Ad Commandinum fere confugerunt, qui postea Elementa interpretati sunt, velut Simson ad eius interpretationem annexit adnotationes suas criticas, quarum p. LIX mentionem fecimus.

Eadem interpretatio Commandini etiam in editionem Oxoniensem transiit (praef. „traductionem plerumque secuti sumus Federici Commandini, at infinitis in locis castigatam, praecipue ex libris clarissimi Edvardi Bernhardi ${ }^{1}$ ) Astronomiae olim professoris Saviliani in bibliotheca Bodleiana asservatis"), quam curavit Dav. Gregorius, Oxon. 1703 fol., et quae ad hunc diem sola est editio operum omnium Euclidis. de subsidiis huius editionis ita Gregorius in praef.: ,,primo" inquit ,textum Graecum quod attinet, ut is quam emendatissimus et castigatissimus prodiret, modis omnibus curavimus, adhibitis, prout opus esset, in consilium mss. codicibus haud paucis melioris notae, quos in hunc ipsissimum usum Academiae pridem legarat magnus Savilius, ut et castigationibus eius propria manu adscriptis ad marginem editionis Hervagianae. accessit singularis et nunquam satis praedicanda amicissimi D. Ioannis Hudsoni S. T. P. protobibliothecarii Bodleiani industria in expoliendis Graecis hisce .... textum Hervagianum ante paullo, quam in typographorum manus traderetur, accurate interpungendum et distinguendum curavit, Latina cum Graecis per totum, in elementis praesertim ac Datis, summa fide contulit. ubi ea a se invicem discrepantia deprehenderentur, vel

1) † 1697 ; hic vir corpus mathematicorum veterum XIV voluminibus comprehensum edere voluit, quorum conspectum dedit Fabricius, Bibl. Gr. II p. 564 sq. vol. I comprehensurum erat Euclidis Elementorum libb. XV ,,iuxta editionem Graecam Basileae 1533 collatam cum Mss. Gr. Bodl. Arch. B 25 et Bodl. S. 4. 9". 


\section{PROLEGOMENA CRITICA}

etiam Graecum ipsum suspectum haberetur, consulti illico mss. codices, quorum lectio, si cum Latinis congrueret, ad marginem adscripta exstabat, sin minus, apposita stellula, ut exinde iudicandi occasio mihi daretur, utra demum lectio Geometricis rationibus magis conveniret." iam hinc apparet, non codicem aliquem, sed editionem principem Hervagii fundamentum esse editionis Oxoniensis, et codices ibi tantum inspectos esse, ubi Hudsono aliqua de causa suspecta esset scriptura editionis Basil. et hoc confirmatur emendationibus adnotationibusque Gregorii perlustratis. nam cum plerumque editionem Basil. sequatur, hic illic in imo mg. ad. notat, aut aliquid e codd. receptum esse (velut p. 225 in X 16 ed. meae), aut aliquid in codd. - nam plerumque de compluribus dicit $^{1}$ ) - omissum esse recte (velut p. 175 in VIII 5; p. 196 in IX 12 ; p. 201 in IX 19 ; p. 206 in IX 33 ; p. 218 in X 9 , alibi) vel perperam (p. 305 ,,deest in cod. ms."), aut omnino aliter in codd. legi (velut p. 97 in V 4; p. 221 in X 11; notabilis est locus p. 251 , in

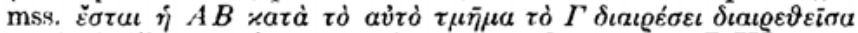
$\varkappa a \tau \dot{\alpha} \tau \dot{o} A^{\prime \prime}$, quae fere est scriptura mendosa codicis $\mathbf{P}$ III p. 69 , 7. 8); inter(hum de suo scripturam edit. Basil. mutat, velut p. 82 (IV 5 coroll.), p. 220 (X 10-11 permutat); p. 329 (XI 1 , hic in

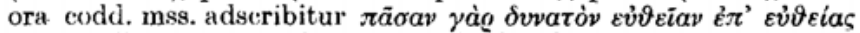
$\dot{\varepsilon} \times \beta a \lambda \varepsilon \bar{c} \nu^{\text {") }}$ miro modo cum ed. Basil. consentit (v. supra p. LXXXII); cf. etiam p. 337.

Quibus codicibus usus sit, non reperi; codices Savilianos Oxonii in Bodleiana non vidi.

Post Gregorium nulla editio Graeca in lucem prodiit ante Peyrardum, qui Parisiis a. 1814 - 1818 tribus voluminibus in 4 to Elementa et Data edidit. ${ }^{2}$ ) is enim, cum a. 1808 iussu Napoleonis I e bibliothecis Italiae optimi codices eligerentur et Parisios mitterentur, impetravit, ut e bibliotheca Vaticana suum in usum a legato Gallorum com. de Peluse codices antiquissimi Elementorum 190 ct 1038 Parisios mitterentur (etiam Vat. 204 eodem tempore Parisiis fuit; sed omnes tres a. 1814 possessoribus legitimis restituti sunt; errat Weissenborn, Philol. Anz. XV 36), et cum prae. stantiam cod. Vat. 190 perspexisset, consilium cepit opera Euclidis genuina Graece Latine Francogallice edendi ope huius codicis (v. praef. eius I p. XII). multa inde in textum recepit, in appendice conspectum scripturae ed. Oxoniensis et codicis 190 dedit, hic illic scripturas Vat. 1038 et XXI codd. Paris. (v. praef. I p. XXVIIIsq.) enotavit. ita viam Elementa emendandi monstravit, sed is quoque iniuria editionem Basil., quam fundamentum esse editionis Oxon. recte intellexerat (praef. I p. XII), e codd. emendandam potius quam prorsus abiiciendam putavit,

1) P. 187 codicem Bodleianum nominat, p. 256 codicem Savjlianum.

2) Iam a. 1804 interpretationem Francogallicam Elementorum ediderat libb. I -IV, XI, XII; ed. secunda ib. 1809 etiam lib. V et X l continet; usus est ed. Oxon. et Simsono.

\section{LXXXVIII}




\section{PROLEGOMENA CRITICA}

ut textus Elementorum novo fundamento constitueretur. ex editoribus posterioribus I. G. Camerer, qui cum Haubero Elem. I - VI edidit duobus voluminibus Berolini 1824-1825 (cum interpr. Lat. et commentario) et Neidius (Elem. I - VI, XI, XII cum glossario Halis Sax. 1825) a Peyrardo pendent, E. F. August (Elem. I - XIII Berolini 1826 - I829) pressius vestigia codicis P sequitur quam Peyrardus, cuius apparatum in appendice dedit non diligentissime, et cod. Vindob. V (v. vol. I p. 309; II p. 309) inspexit saltim; praeterea Proclum respexit (I p. XII; quos ib. p. XIII commemorat codd. Monac. tres, Elementa non continent).

De interpretationibus commentariisque recentioribus paene innumeris omnium fere linguarum non dico, quippe quae saepe ad interpretationem aliquam Latinam facta sint; certe nova subsidia critica nec habuerunt nee quaesiverunt, cum aliud iis propositum esset. 
